Supporting Information for

\title{
Iron-Catalyzed $E$-Selective Dehydrogenative Borylation of Vinylarenes with Pinacolborane
}

Chao Wang, Caizhi Wu, and Shaozhong Ge*

Department of Chemistry, National University of Singapore, 3 Science Drive 3, Singapore 117543 , Singapore

Email: chmgsh@nus.edu.sg

Table of Contents

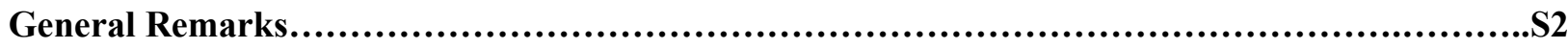

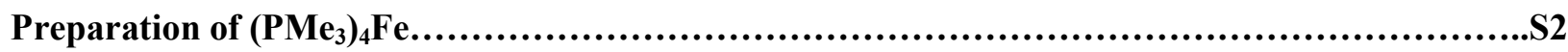

Screening of Other Conditions for Fe-catalyzed Dehydrogenative Borylation........................S3

General Procedure for the Fe-catalyzed Dehydrogenative Borylation of Terminal Vinylarenes with

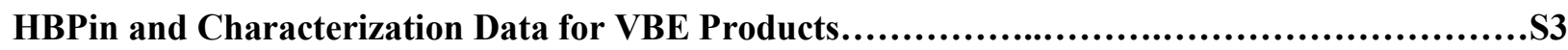

Dehydrogenative Borylation on 10.0 mmol scale................................................

One-pot Procedure for the Halogenation of Vinylarenes............................................

One-pot Procedure for the Iodination of Vinylarenes........................................S10

One-pot Procedure for the Synthesis of Allylic Boronate Ester.................................S10

One-pot Procedure for the Synthesis of $\alpha$-Aryl Aldehydes......................................S11

One-pot Procedure for the Synthesis of Stilbenes...............................................S11

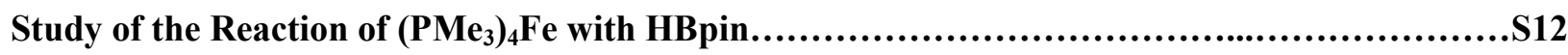

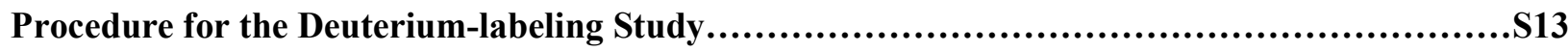

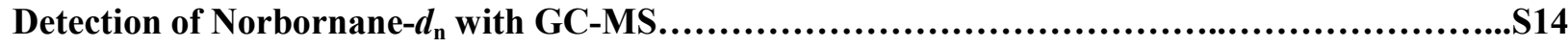

Isomerization Experiment of $Z$-1 a under the Standard Reaction Conditions........................S15

Competition Experiment between Vinylarene and $\boldsymbol{\beta}, \boldsymbol{\beta}$-2D-Vinylarene...........................S15

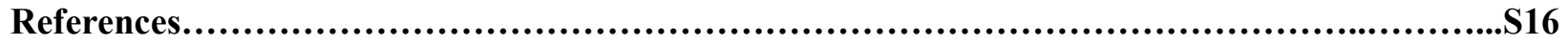

NMR Spectra (including 2D NOESY of compounds 1a, 1e, 1p, 1q) ..............................S17 


\section{General Remarks.}

All the manipulations were performed in a nitrogen-filled glovebox, unless mentioned otherwise. THF, toluene, and hexane were purified by passing the degassed solvents $\left(\mathrm{N}_{2}\right)$ through a column of activated alumina (solvent purification system purchased from Innovative Technologies, Newburyport, MA). The following chemicals were purchased and used as received: Pinacolborane (HBPin) (97\%, Sigma-Aldrich), $\mathrm{PMe}_{3}$ (1.0 M in THF) (Sigma-Aldrich), DMPE (97\%, Sigma-Aldrich), NaHBEt3 (1.0 M in THF) (SigmaAldrich). $\mathrm{PNN}^{1}$, $(\mathrm{PNN}) \mathrm{FeCl}_{2}{ }^{2}$, (DMPE) ${ }_{2} \mathrm{FeCl}_{2}{ }^{3}$, and $\left(\mathrm{PMe}_{3}\right)_{2} \mathrm{FeCl}_{2}{ }^{4}$ were prepared according to previously reported procedures. All other reagents and solvents were purchased from commercial sources and used without purification.

${ }^{1} \mathrm{H},{ }^{13} \mathrm{C}$, and ${ }^{11} \mathrm{~B}$ NMR spectra were recorded using a Varian $400 \mathrm{MHz}, 500 \mathrm{MHz}$, or $600 \mathrm{MHz} \mathrm{NMR}$ spectrometer. ${ }^{1} \mathrm{H}$ NMR and ${ }^{13} \mathrm{C}$ NMR spectra were referenced to resonances of the residual protons in the deuterated solvents. ${ }^{11} \mathrm{~B}$ NMR chemical shifts were referenced to an external $\mathrm{BF}_{3}-\mathrm{Et}_{2} \mathrm{O}$ standard. Multiplicities are recorded as: $\mathrm{s}=$ singlet, $\mathrm{d}=$ doublet, $\mathrm{t}=$ triplet, $\mathrm{dd}=$ doublet of doublets, $\mathrm{br}=$ broad singlet, $\mathrm{m}=$ multiplet. The resonances corresponding to the carbon attached to boron were not observed for the dehydrogenative borylation products in the ${ }^{13} \mathrm{C}$ NMR spectra. GC analysis was acquired on Agilent 6850 gas chromatograph equipped with a flame-ionization detector. GC-MS analysis was performed on Shimadzu GC-2010 gas chromatograph coupled to an Shimadzu QP2010 mass selective detector. HR-MS analyses were performed at a Thermo Scientific Exactive (APCI).

$\alpha$-Methylstyrene, $\alpha$-phenylstyrene, 2,4-diphenyl-4-methyl-1-penteneand and monosubstituted vinylarenes were purchased and used as received. All other alkenes were prepared by Wittig olefination of the corresponding ketones according to previously reported procedures. ${ }^{5,6}$

\section{Preparation of $\left(\mathrm{PMe}_{3}\right)_{4} \mathrm{Fe}$}

$\left(\mathrm{PMe}_{3}\right)_{4} \mathrm{Fe}$ was prepared by following a modified procedure described by Guan. ${ }^{7}$ In an $\mathrm{N}_{2}$-filled dry box, anhydrous $\mathrm{FeCl}_{2}(1.0 \mathrm{~g}, 7.9 \mathrm{mmol})$ and $\mathrm{Mg}$ turnings $(1.5 \mathrm{~g}, 61.7 \mathrm{mmol})$ was added to round bottom flask with some broken glass to activate the Mg turnings. A $1.0 \mathrm{M}$ solution of $\mathrm{PMe}_{3}$ in THF $(39.3 \mathrm{~mL}, 39.3$ mmol) was added under room temperature. The resulting mixture was stirred for $6 \mathrm{~h}$ at room temperature as its color changed from green to dark yellow. The volatiles were removed under vacuum, and the remaining solid was treated with pentane $(30 \mathrm{~mL})$. After filtration, removal of pentane from the filtrate under vacuum produced $\left(\mathrm{PMe}_{3}\right)_{4} \mathrm{Fe}$ as a yellow solid $(2.36 \mathrm{~g}, 83 \%$ yield). The NMR spectrum data are consistent with those reported in the literature. ${ }^{8}{ }^{1} \mathrm{H}$ NMR $\left(500 \mathrm{MHz}, \mathrm{C}_{6} \mathrm{D}_{6}\right) \delta 1.71-1.06(\mathrm{~m}, 33 \mathrm{H}),-0.74(\mathrm{~d}$, $J=158.2 \mathrm{~Hz}, 2 \mathrm{H}),-13.49(\mathrm{~m}, 1 \mathrm{H}) ;{ }^{31} \mathrm{P}$ NMR $\left(202 \mathrm{MHz}, \mathrm{CDCl}_{3}\right) \delta 35.3(\mathrm{dt}), 29.2(\mathrm{dt}), 22.2(\mathrm{q}),-18.3(\mathrm{q})$. 
Screening of other conditions for Fe-catalyzed dehydrogenative borylation ${ }^{\mathrm{a}}$

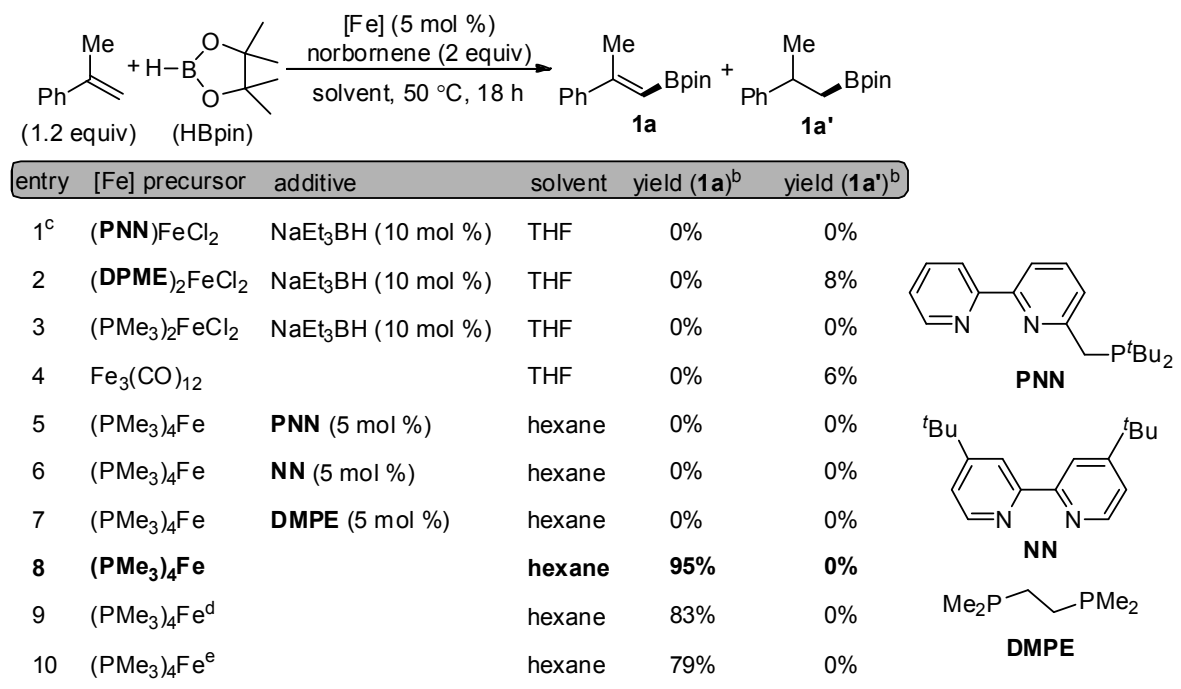

${ }^{a}$ Conditions: $\alpha$-methylstyrene $(0.600 \mathrm{mmol})$, HBpin $(0.500 \mathrm{mmol})$, iron precursor $(5 \mathrm{~mol} \%, 25.0 \mu \mathrm{mol})$, solvent $(1$ $\mathrm{mL}$ ), $50{ }^{\circ} \mathrm{C}, 18 \mathrm{~h} .{ }^{b}$ Determined by GC analysis with dodecane as the internal standard. ${ }^{\circ}$ Hydroboration of norbornene occurred in low GC yields (entry 1: $40 \%$ and entry $4: 22 \%$ ). ${ }^{\mathrm{d}} 3 \mathrm{~mol} \%$ catalyst. ${ }^{\mathrm{e}}$ At room temperature.

\section{General procedure for the Fe-catalyzed dehydrogenative borylation of terminal vinylarenes with HBPin}

In an $\mathrm{N}_{2}$-filled dry box, norbornene $(94.2 \mathrm{mg}, 1.00 \mathrm{mmol})$, HBPin $(73 \mu \mathrm{L}, 0.500 \mathrm{mmol})$, and vinylarenes $(0.600 \mathrm{mmol})$ were added to a $4-\mathrm{mL}$ screw-capped vial containing a magnetic stirring bar. A solution of $\mathrm{Fe}\left(\mathrm{PMe}_{3}\right)_{4}$ in hexane $(1 \mathrm{~mL})$ was added to the mixture. The vial was sealed with a cap containing a PTFE septum and removed from the dry box. The reaction mixture was stirred at $50{ }^{\circ} \mathrm{C}$ for $18 \mathrm{~h}$ and then cooled to room temperature. The mixture was concentrated under vacuum and the crude product was purified by flash chromatography on silica with a mixture of ethyl acetate and hexane as eluent. The conditions for flash chromatography and data for characterization of the products are listed below.

\section{(E)-4,4,5,5-Tetramethyl-2-(2-phenylprop-1-en-1-yl)-1,3,2-dioxaborolane (1a)}

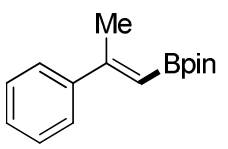

The title compound was isolated $(114 \mathrm{mg}, 0.467 \mathrm{mmol}, 93 \%)$ as a colorless oil after chromatography on silica with ethyl acetate/hexane (1:40). ${ }^{1} \mathrm{H}$ NMR $\left(300 \mathrm{MHz}, \mathrm{CDCl}_{3}\right) \delta$ $7.55(\mathrm{~m}, 2 \mathrm{H}), 7.41-7.28(\mathrm{~m}, 3 \mathrm{H}), 5.81(\mathrm{~s}, 1 \mathrm{H}), 2.46(\mathrm{~s}, 3 \mathrm{H}), 1.36(\mathrm{~s}, 12 \mathrm{H}) ;{ }^{13} \mathrm{C}\left\{{ }^{1} \mathrm{H}\right\} \mathrm{NMR}$ $\left(75 \mathrm{MHz}, \mathrm{CDCl}_{3}\right) \delta 157.9,144.0,128.3,128.0,126.0,83.1,25.0,20.2 ;{ }^{11} \mathrm{~B}$ NMR $(160$ $\left.\mathrm{MHz}, \mathrm{CDCl}_{3}\right) \delta 30.11$. This compound was known. ${ }^{9}$

\section{(E)-4,4,5,5-Tetramethyl-2-(2-(4-(trifluoromethyl)phenyl)prop-1-en-1-yl)-1,3,2-dioxaborolane (1b)}<smiles>O=[W]/C(=C\c1ccccc1)c1ccc(C(F)(F)F)cc1</smiles>

The title compound was isolated $(117 \mathrm{mg}, 0.375 \mathrm{mmol}, 75 \%)$ as a colorless oil after chromatography on silica with ethyl acetate/hexane (1:40). ${ }^{1} \mathrm{H}$ NMR $(500 \mathrm{MHz}$, $\left.\mathrm{CDCl}_{3}\right) \delta 7.57(\mathrm{~s}, 4 \mathrm{H}), 5.79(\mathrm{~s}, 1 \mathrm{H}), 2.41(\mathrm{~s}, 3 \mathrm{H}), 1.32(\mathrm{~s}, 12 \mathrm{H}) ;{ }^{13} \mathrm{C}\left\{{ }^{1} \mathrm{H}\right\} \mathrm{NMR}(126$ $\left.\mathrm{MHz}, \mathrm{CDCl}_{3}\right) \delta 156.3,147.5,130.0\left(\mathrm{q}, J_{\mathrm{C}-\mathrm{F}}=32.8 \mathrm{~Hz}\right), 126.3,125.3\left(\mathrm{q}, J_{\mathrm{C}-\mathrm{F}}=3.7\right.$ $\mathrm{Hz}), 124.8\left(\mathrm{q}, J_{\mathrm{C}-\mathrm{F}}=272 \mathrm{~Hz}\right), 83.3,25.0,20.2 ;{ }^{11} \mathrm{~B}$ NMR (160 MHz, $\left.\mathrm{CDCl}_{3}\right) \delta 29.99$. GC-MS (EI): Calcd for $\mathrm{C}_{16} \mathrm{H}_{20} \mathrm{BF}_{3} \mathrm{O}_{2}: 312.2$, found: 312.2 .

\section{(E)-4,4,5,5-Tetramethyl-2-(2-(p-tolyl)prop-1-en-1-yl)-1,3,2-dioxaborolane (1c)}

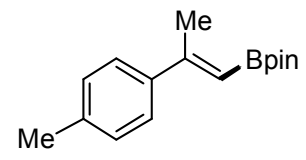

The title compound was isolated (121 $\mathrm{mg}, 0.469 \mathrm{mmol}, 94 \%)$ as a colorless oil after chromatography on silica with ethyl acetate/hexane (1:40). ${ }^{1} \mathrm{H}$ NMR $(500 \mathrm{MHz}$, 
$\left.\mathrm{CDCl}_{3}\right) \delta 7.41(\mathrm{~d}, J=8.2 \mathrm{~Hz}, 2 \mathrm{H}), 7.13(\mathrm{~d}, J=8.0 \mathrm{~Hz}, 2 \mathrm{H}), 5.74(\mathrm{~s}, 1 \mathrm{H}), 2.40(\mathrm{~d}, J=0.5 \mathrm{~Hz}, 3 \mathrm{H}), 2.34(\mathrm{~s}$, $3 \mathrm{H}), 1.31(\mathrm{~s}, 12 \mathrm{H}) ;{ }^{13} \mathrm{C}\left\{{ }^{1} \mathrm{H}\right\} \mathrm{NMR}\left(126 \mathrm{MHz}, \mathrm{CDCl}_{3}\right) \delta 157.8,141.0,137.9,129.0,125.9,83.0,25.0,21.2$, $20.2 ;{ }^{11} \mathrm{~B}$ NMR $\left(160 \mathrm{MHz}, \mathrm{CDCl}_{3}\right) \delta 30.17$. This compound was known. 9

\section{(E)-2-(2-(4-(tert-Butyl)phenyl)prop-1-en-1-yl)-4,4,5,5-tetramethyl-1,3,2-dioxaborolane (1d)}

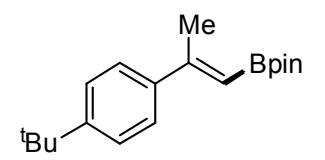

The title compound was isolated (129 $\mathrm{mg}, 0.430 \mathrm{mmol}, 86 \%)$ as a colorless oil after chromatography on silica with ethyl acetate/hexane (1:40). ${ }^{1} \mathrm{H}$ NMR $(300 \mathrm{MHz}$, $\left.\mathrm{CDCl}_{3}\right) \delta 7.48(\mathrm{~d}, J=8.4 \mathrm{~Hz}, 2 \mathrm{H}), 7.37(\mathrm{~d}, J=8.4 \mathrm{~Hz}, 2 \mathrm{H}), 5.79(\mathrm{~s}, 1 \mathrm{H}), 2.43(\mathrm{~s}$, $3 \mathrm{H}), 1.34(\mathrm{~s}+\mathrm{s}, 9 \mathrm{H}+12 \mathrm{H}) ;{ }^{13} \mathrm{C}\left\{{ }^{1} \mathrm{H}\right\} \mathrm{NMR}\left(75 \mathrm{MHz}, \mathrm{CDCl}_{3}\right) \delta 157.6,151.1,140.9$, 125.7, 125.2, 83.0, 34.6, 31.4, 25.0, 20.1; ${ }^{11} \mathrm{~B}$ NMR (160 MHz, $\left.\mathrm{CDCl}_{3}\right) \delta 30.17$. This compound was known. ${ }^{9}$

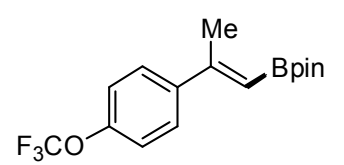

\section{(E)-4,4,5,5-Tetramethyl-2-(2-(4-(trifluoromethoxy)phenyl)prop-1-en-1-yl)- 1,3,2-dioxaborolane (1e)}

The title compound was isolated $(141 \mathrm{mg}, 0.430 \mathrm{mmol}, 86 \%)$ as a colorless oil after chromatography on silica with ethyl acetate/hexane (1:30). ${ }^{1} \mathrm{H}$ NMR (300 $\left.\mathrm{MHz}, \mathrm{CDCl}_{3}\right) \delta 7.60-7.39(\mathrm{~m}, 2 \mathrm{H}), 7.16(\mathrm{~d}, J=8.2 \mathrm{~Hz}, 2 \mathrm{H}), 5.73(\mathrm{~d}, J=0.7 \mathrm{~Hz}$, $1 \mathrm{H}), 2.39(\mathrm{~s}, 3 \mathrm{H}), 1.31(\mathrm{~s}, 12 \mathrm{H}) ;{ }^{13} \mathrm{C}\left\{{ }^{1} \mathrm{H}\right\} \mathrm{NMR}\left(75 \mathrm{MHz}, \mathrm{CDCl}_{3}\right) \delta 156.0,148.8,142.4,127.1,120.5(\mathrm{q}$, $\left.J_{\mathrm{CF}}=255 \mathrm{~Hz}\right), 120.4,83.0,25.0,20.0 ;{ }^{11} \mathrm{~B} \mathrm{NMR}\left(160 \mathrm{MHz}, \mathrm{CDCl}_{3}\right) \delta 30.00$. HRMS $\left(\mathrm{APCI}^{+}\right) \mathrm{m} / \mathrm{z}$ calcd for $\mathrm{C}_{16} \mathrm{H}_{21} \mathrm{BF}_{3} \mathrm{O}_{3}, \mathrm{M}+\mathrm{H}^{+}$329.1533, found: 329.1530

\section{(E)-4,4,5,5-Tetramethyl-2-(2-(4-(methylsulfonyl)phenyl)prop-1-en-1-yl)-1,3,2-dioxaborolane (1f)}

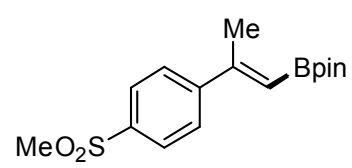

The title compound was isolated (129 $\mathrm{mg}, 0.400 \mathrm{mmol}, 80 \%)$ as a white solid after chromatography on silica with ethyl acetate/hexane (1:10). ${ }^{1} \mathrm{H}$ NMR (300 $\left.\mathrm{MHz}, \mathrm{CDCl}_{3}\right) \delta 7.87(\mathrm{~d}, J=8.4 \mathrm{~Hz}, 2 \mathrm{H}), 7.63(\mathrm{~d}, J=8.4 \mathrm{~Hz}, 2 \mathrm{H}), 5.81(\mathrm{~s}, 1 \mathrm{H})$, $3.04(\mathrm{~s}, 3 \mathrm{H}), 2.40(\mathrm{~s}, 3 \mathrm{H}), 1.31(\mathrm{~s}, 12 \mathrm{H}) ;{ }^{13} \mathrm{C}\left\{{ }^{1} \mathrm{H}\right\} \mathrm{NMR}\left(126 \mathrm{MHz}, \mathrm{CDCl}_{3}\right) \delta$ 155.6, 149.5, 139.6, 127.5, 126.8, 105.1, 83.4, 44.6, 25.0, 20.1; ${ }^{11} \mathrm{~B}$ NMR $\left(160 \mathrm{MHz}, \mathrm{CDCl}_{3}\right) \delta 30.12$. HRMS (APCI ${ }^{+}$) $\mathrm{m} / \mathrm{z}$ calcd for $\mathrm{C}_{16} \mathrm{H}_{24} \mathrm{BO}_{4} \mathrm{~S}, \mathrm{M}+\mathrm{H}^{+} 323.1486$, found: 323.1489 .

\section{(E)-2-(2-(3-Fluorophenyl)prop-1-en-1-yl)-4,4,5,5-tetramethyl-1,3,2-dioxaborolane (1g)}

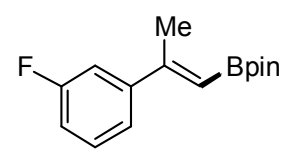

The title compound was isolated $(117 \mathrm{mg}, 0.446 \mathrm{mmol}, 89 \%)$ as a colorless oil after chromatography on silica with ethyl acetate/hexane (1:40). ${ }^{1} \mathrm{H}$ NMR $(500 \mathrm{MHz}$, $\left.\mathrm{CDCl}_{3}\right) \delta 7.33-7.25(\mathrm{~m}, 2 \mathrm{H}), 7.20(\mathrm{~m}, 1 \mathrm{H}), 6.98(\mathrm{~m}, 1 \mathrm{H}), 5.78(\mathrm{~s}, 1 \mathrm{H}), 2.40(\mathrm{~s}, 3 \mathrm{H})$, $1.33(\mathrm{~s}, 12 \mathrm{H}) ;{ }^{13} \mathrm{C}\left\{{ }^{1} \mathrm{H}\right\}$ NMR $\left(126 \mathrm{MHz}, \mathrm{CDCl}_{3}\right) \delta 163.9,162.0,156.3\left(\mathrm{~d}, J_{\mathrm{C}-\mathrm{F}}=1.9\right.$ $\mathrm{Hz}), 146.3\left(\mathrm{~d}, J_{\mathrm{C}-\mathrm{F}}=7.2 \mathrm{~Hz}\right), 129.6\left(\mathrm{~d}, J_{\mathrm{C}-\mathrm{F}}=8.3 \mathrm{~Hz}\right), 121.6\left(\mathrm{~d}, J_{\mathrm{C}-\mathrm{F}}=2.6 \mathrm{~Hz}\right), 114.7\left(\mathrm{~d}, J_{\mathrm{C}-\mathrm{F}}=21.3 \mathrm{~Hz}\right)$, $112.9\left(\mathrm{~d}, J_{\mathrm{C}-\mathrm{F}}=22.1 \mathrm{~Hz}\right), 83.2,25.0,20.1 ;{ }^{11} \mathrm{~B} \mathrm{NMR}\left(160 \mathrm{MHz}, \mathrm{CDCl}_{3}\right) \delta 29.87$. This compound was known. ${ }^{9}$

\section{(E)-2-(2-(3-Methoxyphenyl)prop-1-en-1-yl)-4,4,5,5-tetramethyl-1,3,2-dioxaborolane (1h)}

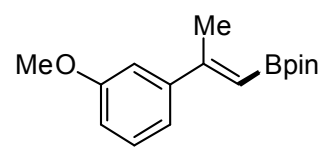

The title compound was isolated (115 $\mathrm{mg}, 0.419 \mathrm{mmol}, 84 \%)$ as a colorless oil after chromatography on silica with ethyl acetate/hexane (1:40). ${ }^{1} \mathrm{H}$ NMR (300 $\left.\mathrm{MHz}, \mathrm{CDCl}_{3}\right) \delta 7.75(\mathrm{t}, J=7.7 \mathrm{~Hz}, 1 \mathrm{H}), 7.67-7.46(\mathrm{~m}, 2 \mathrm{H}), 7.35(\mathrm{~d}, J=7.7 \mathrm{~Hz}$, $1 \mathrm{H}), 6.28(\mathrm{~s}, 1 \mathrm{H}), 4.32(\mathrm{~s}, 3 \mathrm{H}), 2.91(\mathrm{~s}, 3 \mathrm{H}), 1.83(\mathrm{~s}, 12 \mathrm{H}) ;{ }^{13} \mathrm{C}\left\{{ }^{1} \mathrm{H}\right\} \mathrm{NMR}(75 \mathrm{MHz}$, $\left.\mathrm{CDCl}_{3}\right) \delta 159.4,157.6,145.3,129.0,118.3,113.5,111.3,82.9,55.1,24.8,20.1 ;{ }^{11} \mathrm{~B}$ NMR (160 MHz, $\left.\mathrm{CDCl}_{3}\right) \delta 30.01$. HRMS (APCI $) \mathrm{m} / \mathrm{z}$ calcd for $\mathrm{C}_{16} \mathrm{H}_{24} \mathrm{BO}_{3}, \mathrm{M}+\mathrm{H}^{+} 275.1816$, found: 275.1809.

\section{(E)-2-(2-(3,5-Dimethylphenyl)prop-1-en-1-yl)-4,4,5,5-tetramethyl-1,3,2-dioxaborolane (1i)}

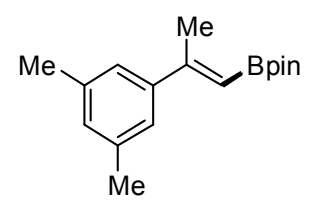

The title compound was isolated (108 $\mathrm{mg}, 0.397 \mathrm{mmol}, 79 \%)$ as a colorless oil after chromatography on silica with ethyl acetate/hexane (1:40). ${ }^{1} \mathrm{H}$ NMR (300 MHz, 
$\left.\mathrm{CDCl}_{3}\right) \delta 7.13(\mathrm{~s}, 2 \mathrm{H}), 6.94(\mathrm{~s}, 1 \mathrm{H}), 5.74(\mathrm{~s}, 1 \mathrm{H}), 2.40(\mathrm{~s}, 3 \mathrm{H}), 2.32(\mathrm{~s}, 6 \mathrm{H}), 1.32(\mathrm{~s}, 12 \mathrm{H}),{ }^{13} \mathrm{C}\left\{{ }^{1} \mathrm{H}\right\} \mathrm{NMR}$ $\left(75 \mathrm{MHz}, \mathrm{CDCl}_{3}\right) \delta 158.3,144.1,137.6,129.7,123.9,83.0,25.0,21.5,20.3 ;{ }^{11} \mathrm{~B} \mathrm{NMR}\left(160 \mathrm{MHz}, \mathrm{CDCl}_{3}\right)$ $\delta$ 30.05. HRMS (APCI $\left.{ }^{+}\right) \mathrm{m} / \mathrm{z}$ calcd for $\mathrm{C}_{17} \mathrm{H}_{26} \mathrm{BO}_{2}, \mathrm{M}+\mathrm{H}^{+}$273.2026, found: 273.2028 .

\section{(E)-2-(2-(Benzo[d][1,3] dioxol-5-yl)prop-1-en-1-yl)-4,4,5,5-tetramethyl-1,3,2-dioxaborolane (1j)}

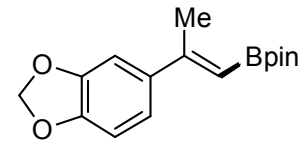

The title compound was isolated (120 $\mathrm{mg}, 0.416 \mathrm{mmol}, 83 \%)$ as a colorless oil after chromatography on silica with ethyl acetate/hexane (1:30). ${ }^{1} \mathrm{H}$ NMR $(300 \mathrm{MHz}$, $\left.\mathrm{CDCl}_{3}\right) \delta 7.01(\mathrm{~m}, 2 \mathrm{H}), 6.75(\mathrm{~d}, J=8.7 \mathrm{~Hz}, 1 \mathrm{H}), 5.95(\mathrm{~s}, 2 \mathrm{H}), 5.66(\mathrm{~s}, 1 \mathrm{H}), 2.36(\mathrm{~s}$, $3 \mathrm{H}), 1.30(\mathrm{~s}, 12 \mathrm{H}) ;{ }^{13} \mathrm{C}\left\{{ }^{1} \mathrm{H}\right\}$ NMR $\left(75 \mathrm{MHz}, \mathrm{CDCl}_{3}\right) \delta 157.2,147.8,138.3,128.9$, 119.9, 107.9, 106.5, 101.2, 83.0, 25.0, 20.3; ${ }^{11} \mathrm{~B}$ NMR (160 MHz, $\left.\mathrm{CDCl}_{3}\right) \delta 27.03 . \mathrm{HRMS}\left(\mathrm{APCI}^{+}\right) \mathrm{m} / \mathrm{z}$ calcd for $\mathrm{C}_{16} \mathrm{H}_{22} \mathrm{BO}_{4}, \mathrm{M}+\mathrm{H}^{+}$289.1609, found: 289.1611 .

\section{(E)-4,4,5,5-tetramethyl-2-(2-(4-(prop-1-en-2-yl)phenyl)prop-1-en-1-yl)-1,3,2-dioxaborolane (1k)}

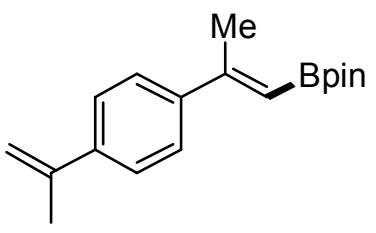

The compound was isolated $(88.1 \mathrm{mg}, 62 \%)$ as a colorless oil after chromatograph on silica with ethyl acetate/hexane (1: 20). ${ }^{1} \mathrm{H}$ NMR $(500 \mathrm{MHz}$, $\left.\mathrm{CDCl}_{3}\right) \delta 7.46(\mathrm{dd}, J=23.8,8.5 \mathrm{~Hz}, 4 \mathrm{H}), 5.80(\mathrm{~s}, 1 \mathrm{H}), 5.40(\mathrm{~s}, 1 \mathrm{H}), 5.12-5.06$ $(\mathrm{m}, 1 \mathrm{H}), 2.42(\mathrm{~d}, J=0.4 \mathrm{~Hz}, 3 \mathrm{H}), 2.16(\mathrm{~s}, 3 \mathrm{H}), 1.32(\mathrm{~s}, 12 \mathrm{H}) ;{ }^{13} \mathrm{C}\left\{{ }^{1} \mathrm{H}\right\} \mathrm{NMR}$ $\left(75 \mathrm{MHz}, \mathrm{CDCl}_{3}\right) \delta 157.3,142.9,140.8,125.8,125.4,112.6,83.1,25.0,21.8$, $20.1 ;{ }^{11} \mathrm{~B}$ NMR $\left(160 \mathrm{MHz}, \mathrm{CDCl}_{3}\right) \delta 30.10$. HRMS $\left(\mathrm{APCI}^{+}\right) \mathrm{m} / \mathrm{z}$ calcd for $\mathrm{C}_{18} \mathrm{H}_{25} \mathrm{BO}_{2}, \mathrm{M}+\mathrm{H}^{+}$284.1948, found: 284.1950.

(E)-tert-Butyldimethyl((4-(1-(4,4,5,5-tetramethyl-1,3,2-dioxaborolan-2-yl)prop-1-en-2-yl)benzyl)oxy)silane (11)<smiles>CCCOc1ccc(C(=C[Pb])c2ccccc2)cc1</smiles>

The title compound was isolated $(138 \mathrm{mg}, 0.355 \mathrm{mmol}, 71 \%)$ as a colorless oil after chromatography on silica with ethyl acetate/hexane (1:30). ${ }^{1} \mathrm{H}$ NMR (500 $\left.\mathrm{MHz}, \mathrm{CDCl}_{3}\right) \delta 7.47(\mathrm{~d}, J=8.2 \mathrm{~Hz}, 2 \mathrm{H}), 7.27(\mathrm{~d}, J=9.9 \mathrm{~Hz}, 2 \mathrm{H}), 5.75(\mathrm{~s}, 1 \mathrm{H})$, $4.73(\mathrm{~s}, 2 \mathrm{H}), 2.40(\mathrm{~s}, 3 \mathrm{H}), 1.31(\mathrm{~s}, 12 \mathrm{H}), 0.94(\mathrm{~s}, 9 \mathrm{H}), 0.10(\mathrm{~s}, 6 \mathrm{H}) ;{ }^{13} \mathrm{C}\left\{{ }^{1} \mathrm{H}\right\}$ NMR $\left(126 \mathrm{MHz}, \mathrm{CDCl}_{3}\right) \delta 157.6,142.4,141.2,125.8,125.7,82.9,64.8,26.0,24.9,20.1,18.4,-5.2 ;{ }^{11} \mathrm{~B}$ NMR $\left(160 \mathrm{MHz}, \mathrm{CDCl}_{3}\right) \delta 30.54$. HRMS $\left(\mathrm{APCI}^{+}\right) \mathrm{m} / \mathrm{z}$ calcd for $\mathrm{C}_{22} \mathrm{H}_{38} \mathrm{BO}_{3} \mathrm{Si}, \mathrm{M}+\mathrm{H}^{+} 389.2682$, found: 389.2686 .

(E)-4,4,5,5-Tetramethyl-2-(2-(4-(2-methyl-1,3-dioxolan-2-yl)phenyl)prop-1-en-1-yl)-1,3,2-dioxaborolane (1m)

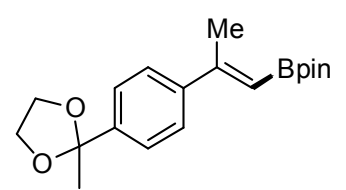

The title compound was isolated (149 $\mathrm{mg}, 0.451 \mathrm{mmol}, 90 \%)$ as a colorless oil after chromatography on silica with ethyl acetate/hexane (1:30). ${ }^{1} \mathrm{H}$ NMR (500 $\left.\mathrm{MHz}, \mathrm{CDCl}_{3}\right) \delta 7.43(\mathrm{~m}, 4 \mathrm{H}), 5.74(\mathrm{~s}, 1 \mathrm{H}), 4.00(\mathrm{t}, J=6.7 \mathrm{~Hz}, 2 \mathrm{H}), 3.75(\mathrm{t}, J=6.8$ $\mathrm{Hz}, 2 \mathrm{H}), 2.38(\mathrm{~s}, 3 \mathrm{H}), 1.63(\mathrm{~s}, 3 \mathrm{H}), 1.29(\mathrm{~s}, 12 \mathrm{H}) ;{ }^{13} \mathrm{C}\left\{{ }^{1} \mathrm{H}\right\}$ NMR $(126 \mathrm{MHz}$, $\left.\mathrm{CDCl}_{3}\right) \delta 157.4,143.4,143.0,125.7,125.2,108.8,83.0,64.5,27.5,24.9,20.1 ;{ }^{11} \mathrm{~B}$ NMR $\left(160 \mathrm{MHz}, \mathrm{CDCl}_{3}\right) \delta$ 29.91. HRMS $\left(\mathrm{APCI}^{+}\right) \mathrm{m} / \mathrm{z}$ calcd for $\mathrm{C}_{19} \mathrm{H}_{28} \mathrm{BO}_{4}, \mathrm{M}+\mathrm{H}^{+} 331.2079$, found: 331.2080 .

\section{(E)-2-(2-(3-(1,3-Dioxolan-2-yl)phenyl)prop-1-en-1-yl)-4,4,5,5-tetramethyl-1,3,2-dioxaborolane (1n)}

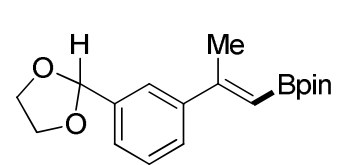

The title compound was isolated $(130 \mathrm{mg}, 0.411 \mathrm{mmol}, 82 \%)$ as a colorless oil after chromatography on silica with ethyl acetate/hexane (1:30). ${ }^{1} \mathrm{H}$ NMR (400 $\left.\mathrm{MHz}, \mathrm{CDCl}_{3}\right) \delta 7.62(\mathrm{t}, J=1.7 \mathrm{~Hz}, 1 \mathrm{H}), 7.52-7.47(\mathrm{~m}, 1 \mathrm{H}), 7.39(\mathrm{~m}, 1 \mathrm{H}), 7.33(\mathrm{t}$, $J=7.6 \mathrm{~Hz}, 1 \mathrm{H}), 5.81(\mathrm{~s}, 1 \mathrm{H}), 5.78(\mathrm{~d}, J=0.9 \mathrm{~Hz}, 1 \mathrm{H}), 4.16-3.99(\mathrm{~m}, 4 \mathrm{H}), 2.41(\mathrm{~d}$, $J=0.9 \mathrm{~Hz}, 3 \mathrm{H}), 1.31(\mathrm{~s}, 12 \mathrm{H}) ;{ }^{13} \mathrm{C}\left\{{ }^{1} \mathrm{H}\right\} \mathrm{NMR}\left(101 \mathrm{MHz}, \mathrm{CDCl}_{3}\right) \delta 157.6,144.2,138.0,128.4,126.8$, 126.2, 124.1, 103.9, 83.1, 65.4, 25.0, 20.2; ${ }^{11} \mathrm{~B}$ NMR (128 MHz, $\left.\mathrm{CDCl}_{3}\right) \delta 30.47$. GC-MS (EI): Calcd for $\mathrm{C}_{18} \mathrm{H}_{25} \mathrm{BO}_{4}$ : 316.2 , found: 316.2 . 
(E)-2,6-diisopropyl-N-(1-(4-((E)-1-(4,4,5,5-tetramethyl-1,3,2-dioxaborolan-2-yl)prop-1-en-2yl)phenyl)ethylidene)aniline (10)

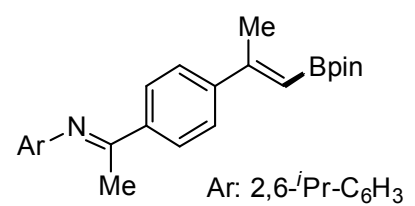

The title compound was isolated (140 $\mathrm{mg}, 0.315 \mathrm{mmol}, 63 \%)$ as a pale yellow solid after chromatography on silica with ethyl acetate/hexane $(1: 30) .{ }^{1} \mathrm{H}$ $\operatorname{NMR}\left(300 \mathrm{MHz}, \mathrm{CDCl}_{3}\right) \delta 8.02(\mathrm{~d}, J=8.1 \mathrm{~Hz}, 2 \mathrm{H}), 7.61(\mathrm{~d}, J=8.4 \mathrm{~Hz}, 2 \mathrm{H})$, $7.20-7.03(\mathrm{~m}, 3 \mathrm{H}), 5.85(\mathrm{~s}, 1 \mathrm{H}), 2.75(\mathrm{~m}, 2 \mathrm{H}), 2.45(\mathrm{~s}, 3 \mathrm{H}), 2.11(\mathrm{~s}, 3 \mathrm{H}), 1.34$ $(\mathrm{s}, 12 \mathrm{H}), 1.14(\mathrm{t}, J=6.9 \mathrm{~Hz}, 12 \mathrm{H}) ;{ }^{13} \mathrm{C}\left\{{ }^{1} \mathrm{H}\right\} \mathrm{NMR}\left(75 \mathrm{MHz}, \mathrm{CDCl}_{3}\right) \delta 164,7$, $156.8,146.0,136.3,127.1,125.9,123.5,122.9,116.6,83.0,28.2,24.8,23.2,22.9,20.0,18.1$; ${ }^{11} \mathrm{~B}$ NMR $\left(160 \mathrm{MHz}, \mathrm{CDCl}_{3}\right) \delta$ 30.61. HRMS $\left(\mathrm{APCI}^{+}\right) \mathrm{m} / \mathrm{z}$ calcd for $\mathrm{C}_{29} \mathrm{H}_{41} \mathrm{BNO}_{2}, \mathrm{M}+\mathrm{H}^{+}$446.3230, found: 446.3235 .

\section{(E)-2-(2-(Furan-2-yl)prop-1-en-1-yl)-4,4,5,5-tetramethyl-1,3,2-dioxaborolane (1p)}

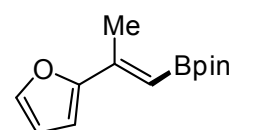

The title compound was isolated $(72.6 \mathrm{mg}, 0.310 \mathrm{mmol}, 62 \%)$ as a colorless oil after chromatography on silica with ethyl acetate/hexane (1:40). ${ }^{1} \mathrm{H}$ NMR $\left(500 \mathrm{MHz}, \mathrm{CDCl}_{3}\right)$ $\delta 7.38(\mathrm{~s}, 1 \mathrm{H}), 6.44(\mathrm{~d}, J=3.3 \mathrm{~Hz}, 1 \mathrm{H}), 6.39(\mathrm{dd}, J=3.3,1.8 \mathrm{~Hz}, 1 \mathrm{H}), 5.92(\mathrm{~s}, 1 \mathrm{H}), 2.28$ $(\mathrm{d}, J=0.7 \mathrm{~Hz}, 3 \mathrm{H}), 1.29(\mathrm{~s}, 12 \mathrm{H}) ;{ }^{13} \mathrm{C}\left\{{ }^{1} \mathrm{H}\right\} \mathrm{NMR}\left(126 \mathrm{MHz}, \mathrm{CDCl}_{3}\right) \delta 156.4,145.6,142.9,111.6,108.1$, 83.0, 25.0, 17.5; ${ }^{11} \mathrm{~B}$ NMR $\left(160 \mathrm{MHz}, \mathrm{CDCl}_{3}\right) \delta 30.22$. HRMS $\left(\mathrm{APCI}^{+}\right) \mathrm{m} / \mathrm{z}$ calcd for $\mathrm{C}_{13} \mathrm{H}_{20} \mathrm{BO}_{3}, \mathrm{M}+\mathrm{H}^{+}$ 235.1502, found: 235.1503 .

\section{(E)-4,4,5,5-Tetramethyl-2-(2-phenylbut-1-en-1-yl)-1,3,2-dioxaborolane (1q)}

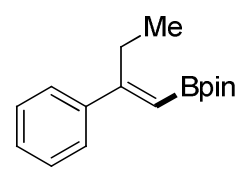

The title compound was isolated (107 $\mathrm{mg}, 0.414 \mathrm{mmol}, 83 \%)$ as a colorless oil after chromatography on silica with ethyl acetate/hexane (1:40). ${ }^{1} \mathrm{H}$ NMR $\left(300 \mathrm{MHz}, \mathrm{CDCl}_{3}\right)$ d 7.49-7.42 (m, 2H), 7.37-7.27 (m, 3H), $5.62(\mathrm{~s}, 1 \mathrm{H}), 2.91(\mathrm{~m}, 2 \mathrm{H}), 1.31(\mathrm{~s}, 12 \mathrm{H}), 1.03$ $(\mathrm{t}, J=7.5 \mathrm{~Hz}, 3 \mathrm{H}) ;{ }^{13} \mathrm{C}\left\{{ }^{1} \mathrm{H}\right\} \mathrm{NMR}\left(75 \mathrm{MHz}, \mathrm{CDCl}_{3}\right) \delta 165.0,143.2,128.3,127.8,126.5$, 83.0, 26.8, 25.0, 14.9; ${ }^{11} \mathrm{~B}$ NMR $\left(160 \mathrm{MHz}, \mathrm{CDCl}_{3}\right) \delta 29.87$. HRMS $\left(\mathrm{APCI}^{+}\right) \mathrm{m} / \mathrm{z}$ calcd for $\mathrm{C}_{16} \mathrm{H}_{24} \mathrm{BO}_{2}$, $\mathrm{M}+\mathrm{H}^{+} 259.1867$, found: 259.1859 .

\section{(E)-4,4,5,5-Tetramethyl-2-(2-phenylpent-1-en-1-yl)-1,3,2-dioxaborolane (1r)}

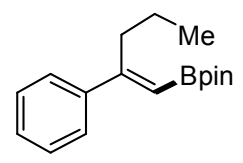

The title compound was isolated $(112 \mathrm{mg}, 0.411 \mathrm{mmol}, 82 \%)$ as a colorless oil after chromatography on silica with ethyl acetate/hexane (1:40). ${ }^{1} \mathrm{H}$ NMR $\left(500 \mathrm{MHz}, \mathrm{CDCl}_{3}\right)$ $\delta$ 7.47-7.36 (m, 2H), 7.33-7.18 (m, 3H), $5.64(\mathrm{~d}, J=1.8 \mathrm{~Hz}, 1 \mathrm{H}), 2.87(\mathrm{~m}, 2 \mathrm{H}), 1.43-$ $1.34(\mathrm{~m}, 2 \mathrm{H}), 1.29(\mathrm{~s}+\mathrm{s}, 6 \mathrm{H}+6 \mathrm{H}), 0.87(\mathrm{~m}, 3 \mathrm{H}),{ }^{13} \mathrm{C}\left\{{ }^{1} \mathrm{H}\right\} \mathrm{NMR}\left(126 \mathrm{MHz}, \mathrm{CDCl}_{3}\right) \delta$ $163.3,143.5,128.3,127.8,126.5,83.0,35.3,25.0,22.9,13.8 ;{ }^{11} \mathrm{~B}$ NMR $\left(160 \mathrm{MHz}, \mathrm{CDCl}_{3}\right) \delta 30.01$. HRMS (APCI ${ }^{+}$) m/z calcd for $\mathrm{C}_{17} \mathrm{H}_{26} \mathrm{BO}_{2}, \mathrm{M}+\mathrm{H}^{+}$273.2023, found: 273.2022.

\section{(E)-4,4,5,5-Tetramethyl-2-(2-phenyloct-1-en-1-yl)-1,3,2-dioxaborolane (1s)}

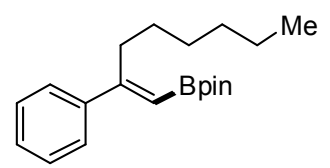

The title compound was isolated $(102 \mathrm{mg}, 0.325 \mathrm{mmol}, 65 \%)$ as a colorless oil after chromatography on silica with ethyl acetate/hexane (1:40). ${ }^{1} \mathrm{H}$ NMR (300 $\left.\mathrm{MHz}, \mathrm{CDCl}_{3}\right) \delta 7.35(\mathrm{dd}, J=7.4,1.3 \mathrm{~Hz}, 2 \mathrm{H}), 7.28-7.11(\mathrm{~m}, 3 \mathrm{H}), 5.54(\mathrm{~s}, 1 \mathrm{H})$, $2.83(\mathrm{~m}, 2 \mathrm{H}), 1.34-1.14(\mathrm{~m}, 20 \mathrm{H}), 0.78(\mathrm{t}, J=6.6 \mathrm{~Hz}, 3 \mathrm{H}) ;{ }^{13} \mathrm{C}\left\{{ }^{1} \mathrm{H}\right\}$ NMR $(75$ $\left.\mathrm{MHz}, \mathrm{CDCl}_{3}\right) \delta 163.7,143.6,128.3,127.8,126.5,83.0,33.5,31.7,29.9,29.2,25.0,22.7,14.2 ;{ }^{11} \mathrm{~B}$ NMR $\left(160 \mathrm{MHz}, \mathrm{CDCl}_{3}\right) \delta 30.03$. This compound was known. ${ }^{10}$

\section{(E)-4,4,5,5-Tetramethyl-2-(4-methyl-2-phenylpent-1-en-1-yl)-1,3,2-dioxaborolane (1t)}

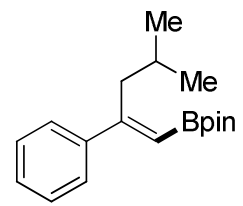

The title compound was isolated (126 $\mathrm{mg}, 0.446 \mathrm{mmol}, 88 \%)$ as a colorless oil after chromatography on silica with ethyl acetate/hexane (1:40). ${ }^{1} \mathrm{H}$ NMR $\left(300 \mathrm{MHz}, \mathrm{CDCl}_{3}\right)$ $\delta$ 7.47-7.35 (m, 2H), 7.34-7.21 (m, 3H), $5.65(\mathrm{~s}, 1 \mathrm{H}), 2.82(\mathrm{~d}, J=7.2 \mathrm{~Hz}, 2 \mathrm{H}), 1.59$ $1.47(\mathrm{~m}, 1 \mathrm{H}), 1.31(\mathrm{~s}, 12 \mathrm{H}), 0.85(\mathrm{~d}, J=6.6 \mathrm{~Hz}, 6 \mathrm{H}),{ }^{13} \mathrm{C}\left\{{ }^{1} \mathrm{H}\right\}$ NMR $\left(126 \mathrm{MHz}, \mathrm{CDCl}_{3}\right)$ $\delta 162.7,143.9,128.3,127.8,126.6,105.1,83.0,42.1,27.7,25.0,22.5 ;{ }^{11} \mathrm{~B}$ NMR $(160$ 
$\left.\mathrm{MHz}, \mathrm{CDCl}_{3}\right) \delta 30.20$. This compound was known. ${ }^{10}$

\section{(E)-4,4,5,5-Tetramethyl-2-(4-methyl-2,4-diphenylpent-1-en-1-yl)-1,3,2-dioxaborolane (1u)}

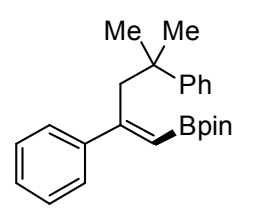

The title compound was isolated $(152 \mathrm{mg}, 0.420 \mathrm{mmol}, 84 \%)$ as a colorless oil after chromatography on silica with ethyl acetate/hexane (1:40). ${ }^{1} \mathrm{H}$ NMR $\left(500 \mathrm{MHz}, \mathrm{CDCl}_{3}\right)$

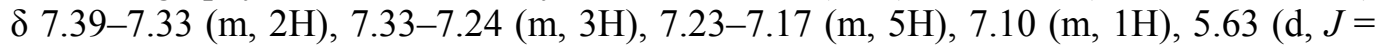
$1.9 \mathrm{~Hz}, 1 \mathrm{H}), 3.29(\mathrm{~d}, J=1.8 \mathrm{~Hz}, 2 \mathrm{H}), 1.34(\mathrm{~s}+\mathrm{s}, 6 \mathrm{H}+6 \mathrm{H}), 1.20(\mathrm{~s}+\mathrm{s}, 3 \mathrm{H}+3 \mathrm{H})$;

${ }^{13} \mathrm{C}\left\{{ }^{1} \mathrm{H}\right\}$ NMR $\left(126 \mathrm{MHz}, \mathrm{CDCl}_{3}\right) \delta 162.0,150.6,145.9,127.9,127.8,127.3,126.7$, 126.0, 125.4, 83.1, 46.8, 38.8, 28.5, 25.1; ${ }^{11} \mathrm{~B}$ NMR (160 MHz, $\left.\mathrm{CDCl}_{3}\right) \delta 29.77$. HRMS $\left(\mathrm{APCI}^{+}\right) \mathrm{m} / \mathrm{z}$ calcd for $\mathrm{C}_{24} \mathrm{H}_{32} \mathrm{BO}_{2}, \mathrm{M}+\mathrm{H}^{+}$363.2494, found: 363.2491 .

\section{2-(2,2-Diphenylvinyl)-4,4,5,5-tetramethyl-1,3,2-dioxaborolane (1v)}

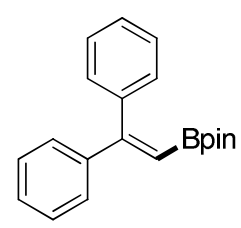

The title compound was isolated $(113 \mathrm{mg}, 0.369 \mathrm{mmol}, 74 \%)$ as a white solid after chromatography on silica with ethyl acetate/hexane (1:30). ${ }^{1} \mathrm{H}$ NMR $\left(300 \mathrm{MHz}, \mathrm{CDCl}_{3}\right)$ $\delta$ 7.38-7.20 (m, 11H), $6.02(\mathrm{~s}, 1 \mathrm{H}), 1.17(\mathrm{~s}, 12 \mathrm{H}) ;{ }^{13} \mathrm{C}\left\{{ }^{1} \mathrm{H}\right\} \mathrm{NMR}\left(75 \mathrm{MHz}, \mathrm{CDCl}_{3}\right) \delta$ $159.7,143.0,141.8,132.3,130.0,129.8,128.2,127.9,127.5,127.5,83.1,24.6 ;{ }^{11} \mathrm{~B}$ NMR $\left(160 \mathrm{MHz}, \mathrm{CDCl}_{3}\right) \delta 30.21$. This compound was known. ${ }^{11}$

\section{2-(2-(4-Methoxyphenyl)-2-phenylvinyl)-4,4,5,5-tetramethyl-1,3,2-dioxaborolane (1w)}

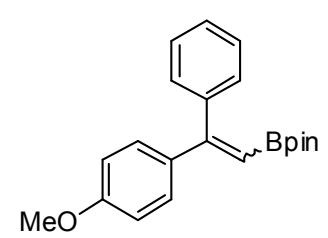

The title compound was isolated (121 $\mathrm{mg}, 0.360 \mathrm{mmol}, 72 \%)$ as a white solid after chromatography on silica with ethyl acetate/hexane (1:30). ${ }^{1} \mathrm{H}$ NMR $(500 \mathrm{MHz}$, $\left.\mathrm{CDCl}_{3}\right) \delta 7.32-7.23(\mathrm{~m}, 6 \mathrm{H}), 7.21-7.18(\mathrm{~m}, 1 \mathrm{H}), 6.85(\mathrm{dd}, J=6.7,4.9 \mathrm{~Hz}, 1.31 \mathrm{H})$, 6.82-6.78 (m, 0.71H), $5.91(\mathrm{~s}, 0.32 \mathrm{H}), 5.88(\mathrm{~s}, 0.63 \mathrm{H}), 3.84(\mathrm{~s}, 2 \mathrm{H}), 3.80(\mathrm{~s}, 1 \mathrm{H})$, $1.19(\mathrm{~s}, 8 \mathrm{H}), 1.14(\mathrm{~s}, 4 \mathrm{H}) ;{ }^{13} \mathrm{C}\left\{{ }^{1} \mathrm{H}\right\} \mathrm{NMR}\left(126 \mathrm{MHz}, \mathrm{CDCl}_{3}\right) \delta$ 159.7, 159.4, 143.7, $142.1,136.4,134.4,131.2,129.9,129.3,128.2,128.0,127.9,127.5,127.5,113.3$, $112.9,83.1,83.0,55.3,55.3,24.7,24.6 ;{ }^{11} \mathrm{~B}$ NMR $\left(160 \mathrm{MHz}, \mathrm{CDCl}_{3}\right) \delta 30.31$. This compound was known. ${ }^{10}$

\section{(E)-4,4,5,5-Tetramethyl-2-styryl-1,3,2-dioxaborolane (2a)}

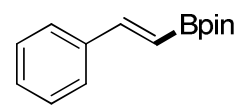

The title compound was isolated (104 $\mathrm{mg}, 0.452 \mathrm{mmol}, 90 \%$ ) as a colorless oil after chromatography on silica with ethyl acetate/hexane (1:40). ${ }^{1} \mathrm{H}$ NMR $\left(300 \mathrm{MHz}, \mathrm{CDCl}_{3}\right)$ $\delta 7.49(\mathrm{~m}, 2 \mathrm{H}), 7.45-7.26(\mathrm{~m}, 4 \mathrm{H}), 6.18(\mathrm{~d}, J=18.4 \mathrm{~Hz}, 1 \mathrm{H}), 1.32(\mathrm{~s}, 12 \mathrm{H}) ;{ }^{13} \mathrm{C}\left\{{ }^{1} \mathrm{H}\right\}$ $\operatorname{NMR}\left(75 \mathrm{MHz}, \mathrm{CDCl}_{3}\right) \delta 149.7,137.7,129.0,128.7,127.2,83.5,24.9 ;{ }^{11} \mathrm{~B} \mathrm{NMR}\left(160 \mathrm{MHz}, \mathrm{CDCl}_{3}\right) \delta$ 30.12. This compound was known. ${ }^{12}$

\section{(E)-4,4,5,5-Tetramethyl-2-(4-methylstyryl)-1,3,2-dioxaborolane (2b)}

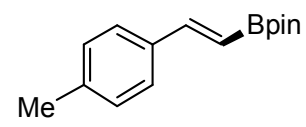

The title compound was isolated $(107 \mathrm{mg}, 0.438 \mathrm{mmol}, 88 \%)$ as a colorless oil after chromatography on silica with ethyl acetate/hexane $(1: 40) .{ }^{1} \mathrm{H}$ NMR $(300 \mathrm{MHz}$, $\left.\mathrm{CDCl}_{3}\right) \delta 7.38(\mathrm{~m}, 3 \mathrm{H}), 7.14(\mathrm{~d}, J=8.0 \mathrm{~Hz}, 2 \mathrm{H}), 6.11(\mathrm{~d}, J=18.5 \mathrm{~Hz}, 1 \mathrm{H}), 2.35(\mathrm{~s}$,

$3 \mathrm{H}), 1.31(\mathrm{~s}, 12 \mathrm{H}) ;{ }^{13} \mathrm{C}\left\{{ }^{1} \mathrm{H}\right\} \mathrm{NMR}\left(126 \mathrm{MHz}, \mathrm{CDCl}_{3}\right) \delta 149.6,139.1,134.9,129.4,127.2,83.4,24.9,21.5$; ${ }^{11} \mathrm{~B}$ NMR $\left(160 \mathrm{MHz}, \mathrm{CDCl}_{3}\right) \delta 30.36$. This compound was known. ${ }^{12}$

\section{(E)-2-(4-(tert-Butyl)styryl)-4,4,5,5-tetramethyl-1,3,2-dioxaborolane (2c)}

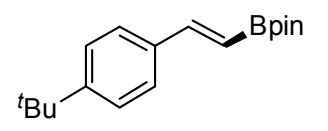

The title compound was isolated $(115 \mathrm{mg}, 0.402 \mathrm{mmol}, 80 \%)$ as a colorless oil after chromatograph on silica with ethyl acetate/hexane (1:40). ${ }^{1} \mathrm{H}$ NMR $(500 \mathrm{MHz}$, $\left.\mathrm{CDCl}_{3}\right) \delta 7.45-7.33(\mathrm{~m}, 5 \mathrm{H}), 6.12(\mathrm{~d}, J=18.4 \mathrm{~Hz}, 1 \mathrm{H}), 1.31(\mathrm{~s}+\mathrm{s}, 9 \mathrm{H}+12 \mathrm{H})$; ${ }^{13} \mathrm{C}\left\{{ }^{1} \mathrm{H}\right\}$ NMR $\left(75 \mathrm{MHz}, \mathrm{CDCl}_{3}\right) \delta 152.2,149.5,134.9,127.0,125.6,83.4,34.8,31.4,24.9 ;{ }^{11} \mathrm{~B}$ NMR $\left(160 \mathrm{MHz}, \mathrm{CDCl}_{3}\right) \delta 30.25$. HRMS $\left(\mathrm{APCI}^{+}\right) \mathrm{m} / \mathrm{z}$ calcd for $\mathrm{C}_{18} \mathrm{H}_{28} \mathrm{BO}_{2}, \mathrm{M}+\mathrm{H}^{+}$287.2180, found: 287.2185. This compound was known. ${ }^{13}$ 


\section{(E)-2-(4-Fluorostyryl)-4,4,5,5-tetramethyl-1,3,2-dioxaborolane (2d)}

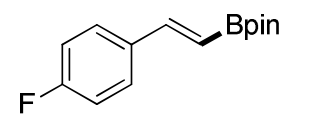

The title compound was isolated $(99.2 \mathrm{mg}, 0.399 \mathrm{mmol}, 80 \%)$ as a colorless oil after chromatography on silica with ethyl acetate/hexane (1:40). ${ }^{1} \mathrm{H}$ NMR $(300 \mathrm{MHz}$, $\left.\mathrm{CDCl}_{3}\right) \delta 7.51-7.40(\mathrm{~m}, 2 \mathrm{H}), 7.35(\mathrm{~d}, J=18.4 \mathrm{~Hz}, 1 \mathrm{H}), 7.01(\mathrm{~m}, 2 \mathrm{H}), 6.07(\mathrm{~d}, J=$ $18.4 \mathrm{~Hz}, 1 \mathrm{H}), 1.31(\mathrm{~s}, 12 \mathrm{H}) ;{ }^{13} \mathrm{C}\left\{{ }^{1} \mathrm{H}\right\} \mathrm{NMR}\left(75 \mathrm{MHz}, \mathrm{CDCl}_{3}\right) \delta 163.3\left(\mathrm{~d}, J_{\mathrm{C}-\mathrm{F}}=247 \mathrm{~Hz}\right), 148.3,133.9$, $128.8\left(\mathrm{~d}, J_{\mathrm{C}-\mathrm{F}}=8.2 \mathrm{~Hz}\right), 115.6\left(\mathrm{~d}, J_{\mathrm{C}-\mathrm{F}}=21.6 \mathrm{~Hz}\right), 83.5,24.9 ;{ }^{11} \mathrm{~B} \mathrm{NMR}\left(160 \mathrm{MHz}, \mathrm{CDCl}_{3}\right) \delta 30.31$. This compound was known. ${ }^{13}$

\section{(E)-2-(4-Methoxystyryl)-4,4,5,5-tetramethyl-1,3,2-dioxaborolane (2e)}

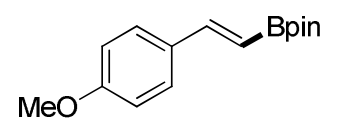

The title compound was isolated (119 $\mathrm{mg}, 0.457 \mathrm{mmol}, 91 \%)$ as a colorless oil after chromatography on silica with ethyl acetate/hexane (1:40). ${ }^{1} \mathrm{H}$ NMR (300 $\left.\mathrm{MHz}, \mathrm{CDCl}_{3}\right) \delta 7.39(\mathrm{~m}, 3 \mathrm{H}), 6.86(\mathrm{~d}, J=8.8 \mathrm{~Hz}, 2 \mathrm{H}), 6.01(\mathrm{~d}, J=18.4 \mathrm{~Hz}, 1 \mathrm{H})$, $3.81(\mathrm{~s}, 3 \mathrm{H}), 1.31(\mathrm{~s}, 12 \mathrm{H}) ;{ }^{13} \mathrm{C}\left\{{ }^{1} \mathrm{H}\right\}$ NMR $\left(75 \mathrm{MHz}, \mathrm{CDCl}_{3}\right) \delta 160.4,149.2,130.6,128.6,114.1,83.3$, $55.4,24.9 ;{ }^{11} \mathrm{~B}$ NMR $\left(160 \mathrm{MHz}, \mathrm{CDCl}_{3}\right) \delta 30.26$. This compound was known. ${ }^{13}$

\section{(E)-N,N-Dimethyl-4-(2-(4,4,5,5-tetramethyl-1,3,2-dioxaborolan-2-yl)vinyl)aniline (2f)}

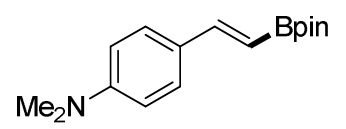

The title compound was isolated $(107 \mathrm{mg}, 0.392 \mathrm{mmol}, 78 \%)$ as a colorless oil after chromatography on silica with ethyl acetate/hexane (1:40). ${ }^{1} \mathrm{H}$ NMR (300 $\left.\mathrm{MHz}, \mathrm{CDCl}_{3}\right) \delta 7.43-7.35(\mathrm{~m}, 2 \mathrm{H}), 7.28(\mathrm{~d}, J=12.9 \mathrm{~Hz}, 1 \mathrm{H}), 6.69(\mathrm{~d}, J=8.1 \mathrm{~Hz}$, $2 \mathrm{H}), 5.93(\mathrm{~d}, J=18.4 \mathrm{~Hz}, 1 \mathrm{H}), 2.98(\mathrm{~s}, 6 \mathrm{H}), 1.30(\mathrm{~d}, J=0.9 \mathrm{~Hz}, 12 \mathrm{H}) ;{ }^{13} \mathrm{C}\left\{{ }^{1} \mathrm{H}\right\} \mathrm{NMR}\left(75 \mathrm{MHz}, \mathrm{CDCl}_{3}\right) \delta$ $149.9,128.5,112.3,83.2,40.6,24.9 ;{ }^{11} \mathrm{~B} \mathrm{NMR}\left(160 \mathrm{MHz}, \mathrm{CDCl}_{3}\right) \delta 30.39$. This compound was known. ${ }^{13}$

\section{(E)-2-(2-([1,1'-Biphenyl]-4-yl)vinyl)-4,4,5,5-tetramethyl-1,3,2-dioxaborolane (2g)}

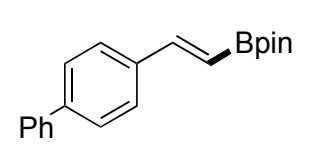

The title compound was isolated $(115 \mathrm{mg}, 0.376 \mathrm{mmol}, 75 \%)$ as a colorless oil after chromatography on silica with ethyl acetate/hexane (1:30). ${ }^{1} \mathrm{H}$ NMR $(500 \mathrm{MHz}$, $\left.\mathrm{CDCl}_{3}\right) \delta 7.66-7.54(\mathrm{~m}, 6 \mathrm{H}), 7.45(\mathrm{~m}, 3 \mathrm{H}), 7.35(\mathrm{t}, J=7.3 \mathrm{~Hz}, 1 \mathrm{H}), 6.23(\mathrm{~d}, J=18.4$ $\mathrm{Hz}, 1 \mathrm{H}), 1.34(\mathrm{~s}, 12 \mathrm{H}) ;{ }^{13} \mathrm{C}\left\{{ }^{1} \mathrm{H}\right\} \mathrm{NMR}\left(126 \mathrm{MHz}, \mathrm{CDCl}_{3}\right) \delta 149.1,141.7,140.7$, 136.6, 128.9, 127.6, 127.6, 127.4, 127.1, 83.5, 24.9; ${ }^{11} \mathrm{~B}$ NMR (160 MHz, $\left.\mathrm{CDCl}_{3}\right) \delta 30.24$. This compound was known. ${ }^{14}$

(E)-4,4,5,5-Tetramethyl-2-(4-(4,4,5,5-tetramethyl-1,3,2-dioxaborolan-2-yl)styryl)-1,3,2dioxaborolane (2h)

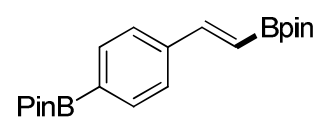

The title compound was isolated (132 $\mathrm{mg}, 0.370 \mathrm{mmol}, 74 \%)$ as a colorless oil after chromatography on silica with ethyl acetate/hexane (1:30). ${ }^{1} \mathrm{H}$ NMR $(500 \mathrm{MHz}$, $\left.\mathrm{CDCl}_{3}\right) \delta 7.77(\mathrm{~d}, J=7.8 \mathrm{~Hz}, 2 \mathrm{H}), 7.48(\mathrm{~d}, J=7.9 \mathrm{~Hz}, 2 \mathrm{H}), 7.40(\mathrm{~d}, J=18.4 \mathrm{~Hz}$, $1 \mathrm{H}), 6.23(\mathrm{~d}, J=18.4 \mathrm{~Hz}, 1 \mathrm{H}), 1.32(\mathrm{~s}+\mathrm{s}, 12 \mathrm{H}+12 \mathrm{H}) ;{ }^{13} \mathrm{C}\left\{{ }^{1} \mathrm{H}\right\} \mathrm{NMR}\left(126 \mathrm{MHz}, \mathrm{CDCl}_{3}\right) \delta 149.5,140.2$, 135.1, 134.9, 126.4, 105.1, 83.9, 83.5, 25.0, 24.9; ${ }^{11} \mathrm{~B}$ NMR (160 MHz, $\left.\mathrm{CDCl}_{3}\right) \delta$ 30.54. GC-MS (EI): Calcd for $\mathrm{C}_{20} \mathrm{H}_{30} \mathrm{~B}_{2} \mathrm{O}_{4}: 356.2$, found: 356.2 .

\section{(E)-2-(2-Fluorostyryl)-4,4,5,5-tetramethyl-1,3,2-dioxaborolane (2i)}

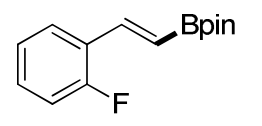

The title compound was isolated (113 $\mathrm{mg}, 0.455 \mathrm{mmol}, 91 \%)$ as a colorless oil after chromatography on silica with ethyl acetate/hexane (1:40). ${ }^{1} \mathrm{H}$ NMR (300 MHz, $\left.\mathrm{CDCl}_{3}\right)$ $\delta 7.49(\mathrm{~m}, 2 \mathrm{H}), 7.38-7.28(\mathrm{~m}, 3 \mathrm{H}), 6.18(\mathrm{~d}, J=18.4 \mathrm{~Hz}, 1 \mathrm{H}), 1.32(\mathrm{~s}, 12 \mathrm{H}) . ;{ }^{13} \mathrm{C}\left\{{ }^{1} \mathrm{H}\right\}$ ${ }^{1} \mathrm{NMR}\left(75 \mathrm{MHz}, \mathrm{CDCl}_{3}\right) \delta 149.6,137.6,129.0,128.7,128.2\left(\mathrm{~d}, J_{\mathrm{C}-\mathrm{F}}=13.2 \mathrm{~Hz}\right), 127.18,83.46,24.93 ;{ }^{11} \mathrm{~B}$ NMR $\left(160 \mathrm{MHz}, \mathrm{CDCl}_{3}\right) \delta 30.19$. This compound was known. ${ }^{15}$

\section{(E)-2-(2,4-Dimethylstyryl)-4,4,5,5-tetramethyl-1,3,2-dioxaborolane (2j)}

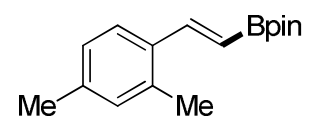

The title compound was isolated $(106 \mathrm{mg}, 0.411 \mathrm{mmol}, 82 \%)$ as a colorless oil after chromatography on silica with ethyl acetate/hexane (1:40). ${ }^{1} \mathrm{H}$ NMR (500 MHz, 
$\left.\mathrm{CDCl}_{3}\right) \delta 7.63(\mathrm{~d}, J=18.3 \mathrm{~Hz}, 1 \mathrm{H}), 7.47(\mathrm{~d}, J=7.9 \mathrm{~Hz}, 1 \mathrm{H}), 7.02-6.94(\mathrm{~m}, 2 \mathrm{H}), 6.05(\mathrm{~d}, J=18.3 \mathrm{~Hz}$, $1 \mathrm{H}), 2.40(\mathrm{~s}, 3 \mathrm{H}), 2.31(\mathrm{~s}, 3 \mathrm{H}), 1.32(\mathrm{~s}, 12 \mathrm{H}) ;{ }^{13} \mathrm{C}\left\{{ }^{1} \mathrm{H}\right\}$ NMR $\left(126 \mathrm{MHz}, \mathrm{CDCl}_{3}\right) \delta 147.2,138.7,136.3$, 134.0, 131.3, 127.0, 125.9, 83.3, 24.9, 21.3, 19.8; ${ }^{11} \mathrm{~B}$ NMR $\left(160 \mathrm{MHz}, \mathrm{CDCl}_{3}\right) \delta 30.23$. This compound was known. ${ }^{13}$

\section{(E)-2-(3-Chlorostyryl)-4,4,5,5-tetramethyl-1,3,2-dioxaborolane (2k)}

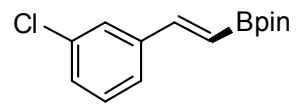

This reaction was conducted with $10 \mathrm{~mol} \%$ of $\left(\mathrm{PMe}_{3}\right)_{4} \mathrm{Fe}$. The title compound was isolated $(67.5 \mathrm{mg}, 0.254 \mathrm{mmol}, 51 \%)$ as a colorless oil after chromatography on silica with ethyl acetate/hexane (1:40). ${ }^{1} \mathrm{H}$ NMR $\left(400 \mathrm{MHz}, \mathrm{CDCl}_{3}\right) \delta 7.38(\mathrm{~d}, J=$ $1.3 \mathrm{~Hz}, 1 \mathrm{H}), 7.27(\mathrm{~m}, 1 \mathrm{H}), 7.23-7.20(\mathrm{~m}, 1 \mathrm{H}), 7.18(\mathrm{~m}, 2 \mathrm{H}), 6.09(\mathrm{~d}, J=18.4 \mathrm{~Hz}, 1 \mathrm{H}), 1.23(\mathrm{~s}, 12 \mathrm{H})$; ${ }^{13} \mathrm{C}\left\{{ }^{1} \mathrm{H}\right\} \mathrm{NMR}\left(126 \mathrm{MHz}, \mathrm{CDCl}_{3}\right) \delta 148.0,139.5,134.7,129.9,128.9,127.1,125.3,83.7,24.9 ;{ }^{11} \mathrm{~B}$ NMR $\left(160 \mathrm{MHz} \mathrm{CDCl}_{3}\right) \delta 30.19$. This compound was known. ${ }^{16}$

\section{(E)-diphenyl(4-(2-(4,4,5,5-tetramethyl-1,3,2-dioxaborolan-2-yl)vinyl)phenyl)phosphine (2l)}

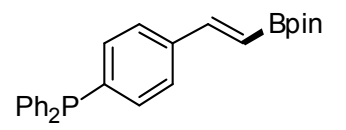

The compound was isolated $(124.3 \mathrm{mg}, 60 \%)$ as a white solid after chromatograph on silica with ethyl acetate/hexane (1: 40). ${ }^{1} \mathrm{H}$ NMR $\left(300 \mathrm{MHz}, \mathrm{CDCl}_{3}\right) \delta 7.44(\mathrm{~m}$, $3 \mathrm{H}), 7.37-7.29(\mathrm{~m}, 12 \mathrm{H}), 6.20(\mathrm{~d}, J=18.4 \mathrm{~Hz}, 1 \mathrm{H}), 1.32(\mathrm{~s}, 12 \mathrm{H}) ;{ }^{13} \mathrm{C}\left\{{ }^{1} \mathrm{H}\right\} \mathrm{NMR}$ $\left(75 \mathrm{MHz}, \mathrm{CDCl}_{3}\right) \delta 148.8,138.2,138.1,137.7,136.9,136.7,133.9,133.7,133.6,133.4,132.1,132.0$, $128.8,128.5,128.4,128.3,127.0,126.9,83.3,24.7 ;{ }^{11} \mathrm{~B}$ NMR $\left(160 \mathrm{MHz}, \mathrm{CDCl}_{3}\right) \delta 30.28 . \mathrm{HRMS}\left(\mathrm{APCI}^{+}\right)$ $\mathrm{m} / \mathrm{z}$ calcd for $\mathrm{C}_{26} \mathrm{H}_{28} \mathrm{BO}_{2} \mathrm{P}, \mathrm{M}+\mathrm{H}^{+}$414.1920, found: 414.1921 .

\section{(E)-Trimethoxy(4-(2-(4,4,5,5-tetramethyl-1,3,2-dioxaborolan-2-yl)vinyl)phenyl)silane (2m)}

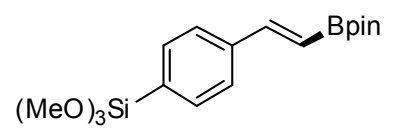

The title compound was isolated (142 $\mathrm{mg}, 0.405 \mathrm{mmol}, 81 \%)$ as a colorless oil after chromatography on silica with ethyl acetate/hexane (1:40). ${ }^{1} \mathrm{H}$ NMR (300 $\left.\mathrm{MHz}, \mathrm{CDCl}_{3}\right) \delta 7.62(\mathrm{~d}, J=7.9 \mathrm{~Hz}, 2 \mathrm{H}), 7.52(\mathrm{~m}, 2 \mathrm{H}), 7.39(\mathrm{~d}, J=18.5 \mathrm{~Hz}$, $1 \mathrm{H}), 6.22(\mathrm{~d}, J=18.4 \mathrm{~Hz}, 1 \mathrm{H}), 3.62(\mathrm{~s}, 9 \mathrm{H}), 1.31(\mathrm{~s}, 12 \mathrm{H}) ;{ }^{13} \mathrm{C}\left\{{ }^{1} \mathrm{H}\right\}$ NMR $\left(75 \mathrm{MHz}, \mathrm{CDCl}_{3}\right) \delta 149.3$, 139.6, 135.2, 130.3, 126.7, 83.6, 51.0, 24.9; ${ }^{11} \mathrm{~B} \mathrm{NMR}$ (160 MHz, $\left.\mathrm{CDCl}_{3}\right) \delta 30.34$. GC-MS (EI): Calcd for $\mathrm{C}_{17} \mathrm{H}_{27} \mathrm{BO}_{5} \mathrm{Si}: 350.2$, found: 350.2 .

\section{(E)-4,4,5,5-Tetramethyl-2-(4-(2-methyl-1,3-dioxolan-2-yl)styryl)-1,3,2-dioxaborolane (2n)}

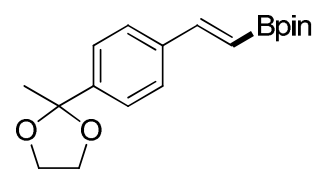

The title compound was isolated (146 $\mathrm{mg}, 0.461 \mathrm{mmol}, 92 \%)$ as a colorless oil after chromatography on silica with ethyl acetate/hexane (1:30). ${ }^{1} \mathrm{H}$ NMR $(500 \mathrm{MHz}$, $\left.\mathrm{CDCl}_{3}\right) \delta 7.52-7.42(\mathrm{~m}, 4 \mathrm{H}), 7.39(\mathrm{~d}, J=18.4 \mathrm{~Hz}, 1 \mathrm{H}), 6.16(\mathrm{~d}, J=18.4 \mathrm{~Hz}, 1 \mathrm{H})$, $4.03(\mathrm{~m}, 2 \mathrm{H}), 3.77(\mathrm{~m}, 2 \mathrm{H}), 1.65(\mathrm{~s}, 3 \mathrm{H}), 1.31(\mathrm{~s}, 12 \mathrm{H}) ;{ }^{13} \mathrm{C}\left\{{ }^{1} \mathrm{H}\right\} \mathrm{NMR}(126 \mathrm{MHz}$, $\left.\mathrm{CDCl}_{3}\right) \delta 149.2,144.1,137.3,127.1,125.7,108.9,83.5,64.6,27.6,25.0 ;{ }^{11} \mathrm{~B}$ NMR $\left(160 \mathrm{MHz}, \mathrm{CDCl}_{3}\right) \delta$ 30.28. HRMS ( $\left.\mathrm{APCI}^{+}\right) \mathrm{m} / \mathrm{z}$ calcd for $\mathrm{C}_{18} \mathrm{H}_{26} \mathrm{BO}_{4}, \mathrm{M}+\mathrm{H}^{+} 317.1922$, found: 317.1923 .

\section{Dehydrogenative Borylation on 10.0 mmol Scale}

In an $\mathrm{N}_{2}$-filled dry box, norbornene (1.88 g, $\left.20.0 \mathrm{mmol}\right)$, HBPin $(1.28 \mathrm{~g}, 10.0 \mathrm{mmol})$, and $\alpha$ methylstyrene $(1.18 \mathrm{~g}, 12 \mathrm{mmol})$ were added to a $100 \mathrm{~mL}$ Schlenk tube containing a magnetic stirring bar. $\mathrm{Fe}\left(\mathrm{PMe}_{3}\right)_{4}(108 \mathrm{mg}, 0.300 \mathrm{mmol})$ in hexane $(20 \mathrm{~mL})$ was added to the mixture. The Schlenk tube was sealed and removed from the dry box. After $18 \mathrm{~h}$ at $50{ }^{\circ} \mathrm{C}$, the reaction mixture was cooled to room temperature and quenched by exposing the solution to air. The resulting solution was concentrated in vacuum and the residue was purified by column chromatography on silica gel to give the product 1a as colorless oil (2.20 g, $9.01 \mathrm{mmol}, 90 \%)$.

\section{One-pot Procedure for the Halogenation of Vinylarenes}

In an $\mathrm{N}_{2}$-filled dry box, norbornene (94.2 $\left.\mathrm{mg}, 1.00 \mathrm{mmol}\right)$, HBPin $(64.0 \mathrm{mg}, 0.500 \mathrm{mmol})$, and $\alpha$ methylstyrene $(70.9 \mathrm{mg}, 0.600 \mathrm{mmol})$ were added to a $20 \mathrm{~mL}$ Schlenk tube containing a magnetic stirring 
bar. $\mathrm{Fe}\left(\mathrm{PMe}_{3}\right)_{4}(9.0 \mathrm{mg}, 25.0 \mu \mathrm{mol})$ in hexane $(1 \mathrm{~mL})$ was added to the mixture. The tube was sealed and removed from the dry box. After $18 \mathrm{~h}$ at $50{ }^{\circ} \mathrm{C}$, the volatile materials were removed under reduced pressure. To the resulting residue were added THF $(0.5 \mathrm{~mL}), \mathrm{H}_{2} \mathrm{O}(0.5 \mathrm{~mL})$ and $\mathrm{CuCl}_{2}$ or $\mathrm{CuBr}_{2}(1 \mathrm{mmol}$, 2 equiv). After $8 \mathrm{~h}$ at $70^{\circ} \mathrm{C}$, the resulting mixture was extracted with EtOAc $(10 \mathrm{~mL})$ and the organic layer was dried over $\mathrm{MgSO}_{4}$. After filtration and evaporation of all volatiles, the residue was purified by column chromatography.

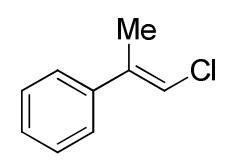

The title compound was isolated $(42.7 \mathrm{mg}, 0.279 \mathrm{mmol}, 56 \%)$ as a colorless oil after chromatography on silica with ethyl acetate/hexane (1:30). ${ }^{1} \mathrm{H} \mathrm{NMR}\left(500 \mathrm{MHz}, \mathrm{CDCl}_{3}\right) \delta$ $7.34(\mathrm{~m}, 4 \mathrm{H}), 7.31-7.27(\mathrm{~m}, 1 \mathrm{H}), 6.32(\mathrm{~d}, J=1.2 \mathrm{~Hz}, 1 \mathrm{H}), 2.22-2.17(\mathrm{~m}, 3 \mathrm{H}) ;{ }^{13} \mathrm{C}\left\{{ }^{1} \mathrm{H}\right\}$ NMR $\left(126 \mathrm{MHz}, \mathrm{CDCl}_{3}\right) \delta 140.5,138.7,128.7,127.9,126.1,115.9,17.0$.<smiles>CC(=CBr)c1ccccc1</smiles>

The title compound was isolated $(78.8 \mathrm{mg}, 0.399 \mathrm{mmol}, 80 \%)$ as a colorless oil after chromatography on silica with ethyl acetate/hexane (1:30). ${ }^{1} \mathrm{H}$ NMR $\left(300 \mathrm{MHz}, \mathrm{CDCl}_{3}\right) \delta$ $7.35(\mathrm{~m}, 5 \mathrm{H}), 6.47(\mathrm{~m}, 1 \mathrm{H}), 2.25(\mathrm{~m}, 3 \mathrm{H}) ;{ }^{13} \mathrm{C}\left\{{ }^{1} \mathrm{H}\right\} \mathrm{NMR}\left(75 \mathrm{MHz}, \mathrm{CDCl}_{3}\right) \delta$ 141.6, 141.1, 128.7, 128.0, 126.1, 105.5, 19.8.

\section{One-pot Procedure for the Iodination of Vinylarene}<smiles>CC(=CI)c1ccccc1</smiles>

In an $\mathrm{N}_{2}$-filled dry box, norbornene (94.2 mg, $\left.1.00 \mathrm{mmol}\right)$, HBPin (64.0 mg, 0.500 $\mathrm{mmol}$ ), and $\alpha$-methylstyrene $(70.9 \mathrm{mg}, 0.600 \mathrm{mmol}$ ) were added to a $20 \mathrm{~mL}$ Schlenk tube containing a magnetic stirring bar. $\mathrm{Fe}\left(\mathrm{PMe}_{3}\right)_{4}(9.0 \mathrm{mg}, 25.0 \mu \mathrm{mol})$ in hexane $(1$ $\mathrm{mL}$ ) was added to the mixture. The tube was sealed and removed from the dry box. After $18 \mathrm{~h}$ at $50{ }^{\circ} \mathrm{C}$, the volatile materials were removed under reduced pressure. To the resulting residue were added THF $(1 \mathrm{~mL})$, and a solution of $\mathrm{NaOH}(0.5 \mathrm{~mL}, 1.5 \mathrm{mmol}, 3 \mathrm{M}$ in water). The resultant mixture was stirred for $10 \mathrm{~min}$ at $23^{\circ} \mathrm{C}$, followed by dropwise addition of a solution of $\mathrm{I}_{2}$ $(250 \mathrm{mg}, 1.00 \mathrm{mmol})$ in THF $(5 \mathrm{~mL})$. After $1 \mathrm{~h}$ at $23^{\circ} \mathrm{C}$, the reaction mixture was quenched with aqueous $\mathrm{Na}_{2} \mathrm{~S}_{2} \mathrm{O}_{3}$ and extracted with ether. The combined organic fraction was washed successively with saturated $\mathrm{NaHCO}_{3}$ and brine, dried over $\mathrm{Na}_{2} \mathrm{SO}_{4}$, filtered, and concentrated. The residue was purified by column chromatography with ethyl acetate/hexane (1:30) to give the title compound as a colorless oil (95.2 $\mathrm{mg}$, $0.390 \mathrm{mmol}, 78 \%)$. ${ }^{1} \mathrm{H}$ NMR $\left(300 \mathrm{MHz}, \mathrm{CDCl}_{3}\right) \delta 7.30-7.09(\mathrm{~m}, 5 \mathrm{H}), 6.43(\mathrm{~d}, J=0.9 \mathrm{~Hz}, 1 \mathrm{H}), 2.19(\mathrm{~d}, J$ $=0.9 \mathrm{~Hz}, 3 \mathrm{H}) ;{ }^{13} \mathrm{C}\left\{{ }^{1} \mathrm{H}\right\} \mathrm{NMR}\left(75 \mathrm{MHz}, \mathrm{CDCl}_{3}\right) \delta 147.3,141.6,128.6,127.9,126.1,79.3,24.5$.

\section{One-pot Procedure for the Synthesis of Allylic Boronate Ester}

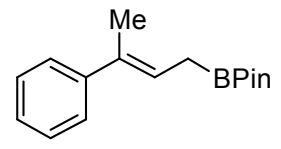

In an $\mathrm{N}_{2}$-filled dry box, norbornene $(94.2 \mathrm{mg}, 1.00 \mathrm{mmol})$, HBPin $(64.0 \mathrm{mg}, 0.500$ mmol), and $\alpha$-methylstyrene (70.9 $\mathrm{mg}, 0.600 \mathrm{mmol}$ ) were added to a $20 \mathrm{~mL}$ Schlenk tube containing a magnetic stirring bar. $\mathrm{Fe}\left(\mathrm{PMe}_{3}\right)_{4}(9.0 \mathrm{mg}, 25.0 \mu \mathrm{mol})$ in hexane (1 $\mathrm{mL}$ ) was added to the mixture. The tube was sealed and removed from the dry box. After $18 \mathrm{~h}$ at $50{ }^{\circ} \mathrm{C}$, the volatile materials were removed under reduced pressure. To the resulting residue were added THF $(4 \mathrm{~mL})$, and bromochloromethane $(67 \mu \mathrm{L}, 1.00 \mathrm{mmol})$ under nitrogen. The resulting clear, colorless solution was cooled to $-78{ }^{\circ} \mathrm{C}$ and treated dropwise with $n$-butyllithium $(2.5 \mathrm{M}$ in hexane, $0.26 \mathrm{~mL}, 0.65 \mathrm{mmol}$ ). After stirring at $-78{ }^{\circ} \mathrm{C}$ for 20 minutes, the cooling bath was removed and the reaction mixture was allowed to slowly warm to room temperature and stirred for additional 3 hours. The resulting mixture was cooled to $0{ }^{\circ} \mathrm{C}$ and water $(2 \mathrm{~mL})$ was slowly added. The mixture was diluted with ethyl acetate $(10 \mathrm{~mL})$ and water $(5 \mathrm{~mL})$. The aqueous layer was extracted with ethyl acetate $(3 \mathrm{x} 5 \mathrm{~mL})$ and the combined organic layers were dried over sodium sulfate, filtered, and concentrated under reduced pressure. The crude material was purified by flash column chromatography with ethyl acetate/hexane (1:30) to give the title compound as a colorless oil $(85.2 \mathrm{mg}, 0.329 \mathrm{mmol}, 66 \%)$. ${ }^{1} \mathrm{H} \mathrm{NMR}(300 \mathrm{MHz}$, $\left.\mathrm{CDCl}_{3}\right) \delta 7.40(\mathrm{~m}, 2 \mathrm{H}), 7.32-7.26(\mathrm{~m}, 2 \mathrm{H}), 7.24-7.16(\mathrm{~m}, 1 \mathrm{H}), 5.94(\mathrm{~m}, 1 \mathrm{H}), 2.04(\mathrm{~s}, 3 \mathrm{H}), 1.85(\mathrm{~d}, J=7.9$ $\mathrm{Hz}, 2 \mathrm{H}), 1.26(\mathrm{~s}, 12 \mathrm{H}) ;{ }^{13} \mathrm{C}\left\{{ }^{1} \mathrm{H}\right\} \mathrm{NMR}\left(75 \mathrm{MHz}, \mathrm{CDCl}_{3}\right) \delta 144.2,134.7,128.2,126.3,125.7,123.2,83.4$, 24.9, 15.8; ${ }^{11} \mathrm{~B}$ NMR $\left(160 \mathrm{MHz}, \mathrm{CDCl}_{3}\right) \delta 33.05$. 
Wang, Wu, and GE, Supporting Information

\section{One-pot Procedure for the Synthesis of $\alpha$-Aryl Aldehydes}

In an $\mathrm{N}_{2}$-filled dry box, norbornene $(94.2 \mathrm{mg}, 1.00 \mathrm{mmol})$, HBPin $(64.0 \mathrm{mg}, 0.500 \mathrm{mmol})$, and $\alpha$ methylstyrene $(70.9 \mathrm{mg}, 0.600 \mathrm{mmol})$ were added to a $20 \mathrm{~mL}$ Schlenk tube containing a magnetic stirring bar. $\mathrm{Fe}\left(\mathrm{PMe}_{3}\right)_{4}(9.0 \mathrm{mg}, 25.0 \mu \mathrm{mol})$ in hexane $(1 \mathrm{~mL})$ was added to the mixture. The tube was sealed and removed from the dry box. After $18 \mathrm{~h}$ at $50{ }^{\circ} \mathrm{C}$, the volatile materials were removed under reduced pressure. To the resulting residue were added sodium peroxoborate tetrahydrate $(92.3 \mathrm{mg}, 0.6 \mathrm{mmol})$, THF $(2 \mathrm{~mL})$, and $\mathrm{H}_{2} \mathrm{O}(2 \mathrm{~mL})$. The solution was cooled to $0{ }^{\circ} \mathrm{C}$ and treated dropwise with $\mathrm{NaOH}(0.33$ $\mathrm{mL}, 1.0 \mathrm{mmol}, 3 \mathrm{M}$ in water) in 10 minutes. After stirring for $1 \mathrm{~h}$ at $0{ }^{\circ} \mathrm{C}, \mathrm{H}_{2} \mathrm{O}(5 \mathrm{~mL})$ was poured into the reaction mixture, and the mixture was extracted with $\mathrm{Et}_{2} \mathrm{O}(5 \mathrm{~mL} \times 3)$. The combined organic layers were washed with brine $(5 \mathrm{~mL})$, dried over $\mathrm{Na}_{2} \mathrm{SO}_{4}$, and filtered. The filtrate was concentrated under reduced pressure. The residue was purified by flash column chromatography.

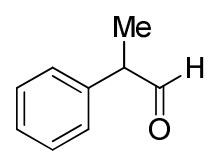

The compound was isolated $(48.3 \mathrm{mg}, 0.360 \mathrm{mmol}, 72 \%)$ as a colorless oil after chromatography on silica with ethyl acetate/hexane (1:30). ${ }^{1} \mathrm{H}$ NMR $\left(500 \mathrm{MHz}, \mathrm{CDCl}_{3}\right) \delta$ $9.69(\mathrm{~d}, J=1.4 \mathrm{~Hz}, 1 \mathrm{H}), 7.42-7.35(\mathrm{~m}, 2 \mathrm{H}), 7.33-7.28(\mathrm{~m}, 1 \mathrm{H}), 7.22(\mathrm{~m}, 2 \mathrm{H}), 3.64(\mathrm{~m}$, $1 \mathrm{H}), 1.45(\mathrm{~m}, 3 \mathrm{H}) ;{ }^{13} \mathrm{C}\left\{{ }^{1} \mathrm{H}\right\} \mathrm{NMR}\left(126 \mathrm{MHz}, \mathrm{CDCl}_{3}\right) \delta 201.1,137.7,129.1,128.3,127.5$,

53.0, 14.6.

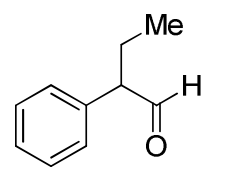

The compound was isolated $(51.9 \mathrm{mg}, 0.350 \mathrm{mmol}, 70 \%)$ as a colorless oil after chromatography on silica with ethyl acetate/hexane (1:30). ${ }^{1} \mathrm{H}$ NMR $\left(500 \mathrm{MHz}, \mathrm{CDCl}_{3}\right) \delta$ $9.68(\mathrm{~d}, J=2.1 \mathrm{~Hz}, 1 \mathrm{H}), 7.38(\mathrm{dd}, J=10.3,4.6 \mathrm{~Hz}, 2 \mathrm{H}), 7.32-7.29(\mathrm{~m}, 1 \mathrm{H}), 7.22-7.15(\mathrm{~m}$, $2 \mathrm{H}), 3.46-3.35(\mathrm{~m}, 1 \mathrm{H}), 2.18-2.04(\mathrm{~m}, 1 \mathrm{H}), 1.86-1.69(\mathrm{~m}, 1 \mathrm{H}), 0.91(\mathrm{~m}, 3 \mathrm{H}) ;{ }^{13} \mathrm{C}\left\{{ }^{1} \mathrm{H}\right\}$ NMR $\left(126 \mathrm{MHz}, \mathrm{CDCl}_{3}\right) \delta 201.0,136.3,129.0,128.8,127.5,60.9,22.9,11.7$.

\section{One-pot Procedure for the Synthesis of Stilbenes}

In an $\mathrm{N}_{2}$-filled dry box, norbornene $(94.2 \mathrm{mg}, 1.00 \mathrm{mmol})$, HBPin $(64.0 \mathrm{mg}, 0.500 \mathrm{mmol})$, and $\alpha$ methylstyrene $(70.9 \mathrm{mg}, 0.600 \mathrm{mmol})$ were added to a $20 \mathrm{~mL}$ Schlenk tube containing a magnetic stirring bar. $\mathrm{Fe}\left(\mathrm{PMe}_{3}\right)_{4}(9.0 \mathrm{mg}, 25.0 \mu \mathrm{mol})$ in hexane $(1 \mathrm{~mL})$ was added to the mixture. The tube was sealed and removed from the dry box. After $18 \mathrm{~h}$ at $50{ }^{\circ} \mathrm{C}$, the volatile materials were removed under reduced pressure. To the resulting residue were added $\mathrm{Pd}(\mathrm{OAc})_{2}(4.5 \mathrm{mg}, 20.0 \mu \mathrm{mol}), \mathrm{P}(t-\mathrm{Bu})_{3} \cdot \mathrm{HBF}_{4}(11.6 \mathrm{mg}$, $40.0 \mu \mathrm{mol}), \mathrm{K}_{2} \mathrm{CO}_{3}(166 \mathrm{mg}, 1.20 \mathrm{mmol})$, bromobenzene $(0.400 \mathrm{mmol}), 1,4$-dioxane $(2.5 \mathrm{~mL})$ and water $(0.5 \mathrm{~mL})$. The vial was sealed and heated at $100{ }^{\circ} \mathrm{C}$ for $5 \mathrm{~h}$. The reaction mixture was cooled to room temperature and extracted with $\mathrm{Et}_{2} \mathrm{O}(10 \mathrm{~mL} \times 3)$. The combined organic layers were washed with brine $(10 \mathrm{~mL})$, dried over $\mathrm{Na}_{2} \mathrm{SO}_{4}$, and filtered. The filtrate was concentrated under reduced pressure. The residue was purified by flash column chromatography.

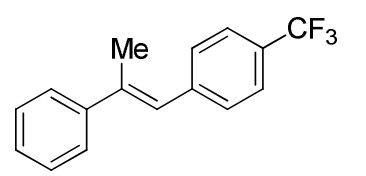

The compound was isolated $(83.9 \mathrm{mg}, 0.320 \mathrm{mmol}, 80 \%)$ as a white solid after chromatography on silica with ethyl acetate/hexane (1: 20). ${ }^{1} \mathrm{H}$ NMR $(500 \mathrm{MHz}$, $\left.\mathrm{CDCl}_{3}\right) \delta 7.64(\mathrm{~d}, J=8.1 \mathrm{~Hz}, 2 \mathrm{H}), 7.54(\mathrm{~d}, J=7.5 \mathrm{~Hz}, 2 \mathrm{H}), 7.47(\mathrm{~d}, J=8.0 \mathrm{~Hz}$, 2H), $7.40(\mathrm{t}, J=7.6 \mathrm{~Hz}, 2 \mathrm{H}), 7.33(\mathrm{t}, J=7.3 \mathrm{~Hz}, 1 \mathrm{H}), 6.85(\mathrm{~s}, 1 \mathrm{H}), 2.29(\mathrm{~s}, 3 \mathrm{H})$;

${ }^{13} \mathrm{C}\left\{{ }^{1} \mathrm{H}\right\} \operatorname{NMR}\left(126 \mathrm{MHz}, \mathrm{CDCl}_{3}\right) \delta 143.5,142.1,139.8,129.5,128.6,127.8,126.5,126.2,125.2\left(J_{\mathrm{C}-\mathrm{F}}=\right.$ $3.8 \mathrm{~Hz}), 123.4,17.7$.

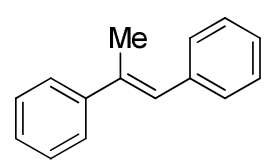

The compound was isolated $(55.2 \mathrm{mg}, 0.284 \mathrm{mmol}, 84 \%)$ as a white solid after chromatography on silica with ethyl acetate/hexane (1: 20). ${ }^{1} \mathrm{H}$ NMR (300 MHz, $\left.\mathrm{CDCl}_{3}\right) \delta 7.62-7.47(\mathrm{~m}, 2 \mathrm{H}), 7.44-7.22(\mathrm{~m}, 8 \mathrm{H}), 6.87(\mathrm{~d}, J=1.1 \mathrm{~Hz}, 1 \mathrm{H}), 2.31(\mathrm{~d}, J=$ $1.2 \mathrm{~Hz}, 3 \mathrm{H}) ;{ }^{13} \mathrm{C}\left\{{ }^{1} \mathrm{H}\right\}$ NMR $\left(75 \mathrm{MHz}, \mathrm{CDCl}_{3}\right) \delta 144.1,138.5,137.6,129.3,128.5$, $128.3,127.8,127.3,126.6,126.1,17.6$. 


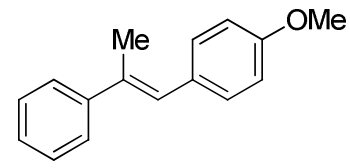

The compound was isolated ( $86.1 \mathrm{mg}, 0.384 \mathrm{mmol}, 96 \%)$ as a white solid after chromatography on silica with ethyl acetate/hexane (1:20). ${ }^{1} \mathrm{H}$ NMR $(300 \mathrm{MHz}$, $\left.\mathrm{CDCl}_{3}\right) \delta 7.63-7.51(\mathrm{~m}, 2 \mathrm{H}), 7.47-7.29(\mathrm{~m}, 5 \mathrm{H}), 7.03-6.92(\mathrm{~m}, 2 \mathrm{H}), 6.86(\mathrm{~s}, 1 \mathrm{H})$, $3.88(\mathrm{~s}, 3 \mathrm{H}), 2.35(\mathrm{~d}, J=1.2 \mathrm{~Hz}, 3 \mathrm{H}) ;{ }^{13} \mathrm{C}\left\{{ }^{1} \mathrm{H}\right\} \mathrm{NMR}\left(75 \mathrm{MHz}, \mathrm{CDCl}_{3}\right) \delta 158.3$,

144.3, 136.0, 131.1, 130.5, 128.4, 127.4, 127.1, 126.0, 113.7, 55.3, 17.6.

\section{Study of the reaction of $\left(\mathrm{PMe}_{3}\right)_{4} \mathrm{Fe}$ with $\mathrm{HBpin}$}

$$
\left(\mathrm{PMe}_{3}\right)_{4} \mathrm{Fe}+\mathrm{HBpin} \stackrel{\text { THF, r.t. }}{\longrightarrow}\left[\left(\mathrm{PMe}_{3}\right)_{4} \mathrm{Fe}(\mathrm{H})(\mathrm{Bpin})\right]
$$

HBpin $(29.3 \mu \mathrm{L}, 0.2 \mathrm{mmol})$ was slowed added to the solution of $\left(\mathrm{PMe}_{3}\right)_{4} \mathrm{Fe}(71.2 \mathrm{mg}, 0.2 \mathrm{mmol})$ in THF $(0.4 \mathrm{~mL})$, and stirred for $2 \mathrm{~h}$. Attempts to isolate the complex $\left(\mathrm{PMe}_{3}\right)_{4} \mathrm{Fe}(\mathrm{H})(\mathrm{Bpin})$ resulted in the formation of ill-defined species. However, HR-MS (ESI) analysis shown cationic fragments of $\left[\left(\mathrm{PMe}_{3}\right)_{4} \mathrm{Fe}(\mathrm{H})\right]^{+}$and $\left[\left(\mathrm{PMe}_{3}\right)_{4} \mathrm{Fe}(\mathrm{Bpin})\right]^{+}$.

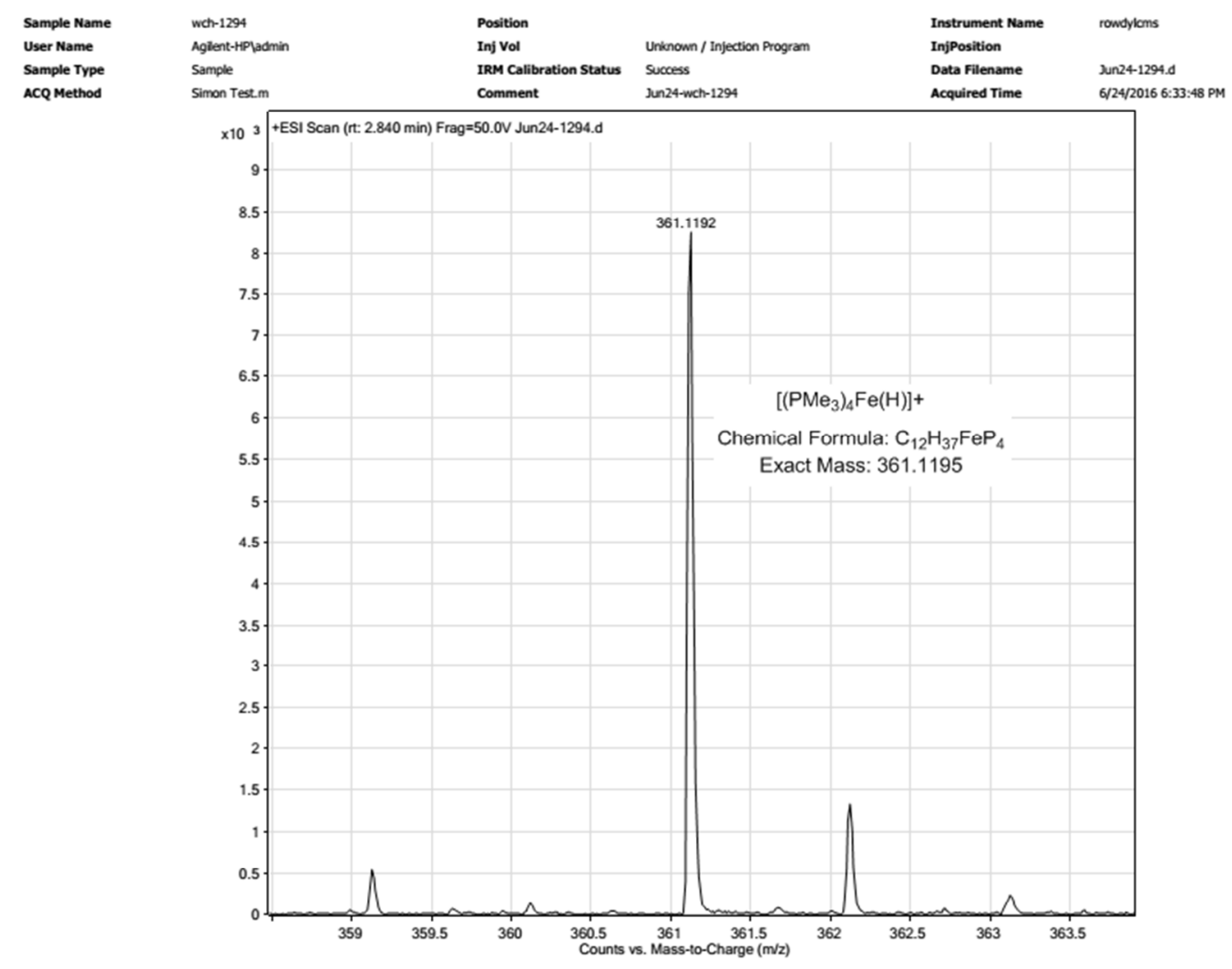




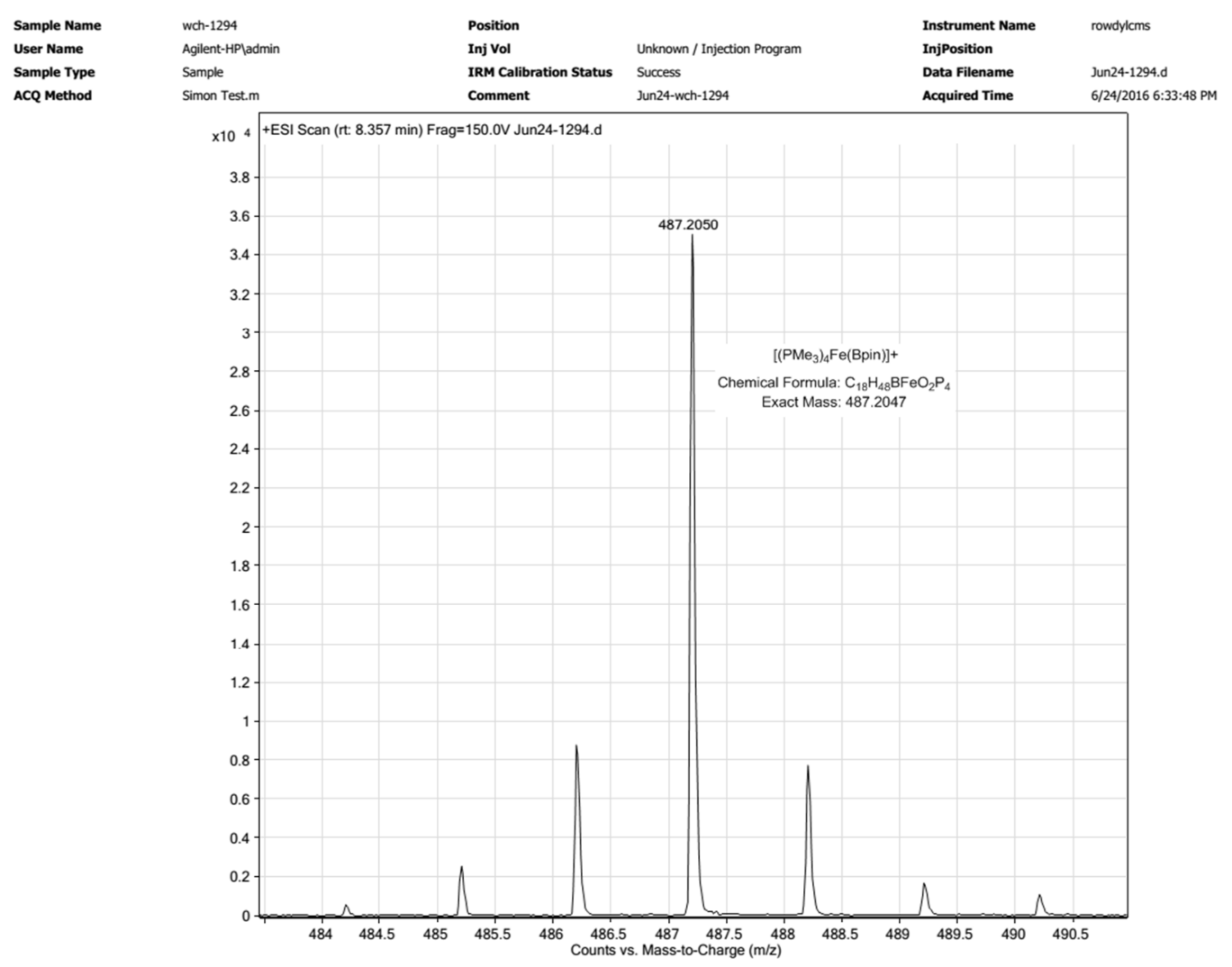

\section{Procedure for the Deuterium-labeling Study}

DBpin was prepared according to a previously reported literature ${ }^{17}$ using hexane instead of THF as solvent, and get a hexane solution $(1.0 \mathrm{M}) .7-d 2$ and $E-7-d 1$ were prepared according to a previously reported procedure ${ }^{18}$ using 4-tert-butylphenylacetylene and $\beta$-D-4-tert-butylphenylacetylene as substrates respectively. E-2,4-diphenyl-4-methyl-1-[1D]-pentene was prepared as the following procedures.

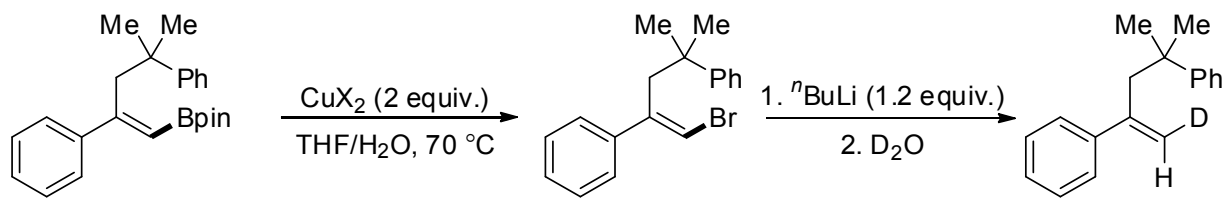

$\mathrm{CuBr}_{2}(2.23 \mathrm{~g}, 10.0 \mathrm{~mol})$ was added to a solution of $\mathbf{1 t}(1.81 \mathrm{~g}, 5.00 \mathrm{mmol})$ in THF $(5 \mathrm{~mL})$ and water $(5$ $\mathrm{mL})$. After stirring at $70{ }^{\circ} \mathrm{C}$ for $18 \mathrm{~h}$, the resulting mixture was extracted with EtOAc $(20.0 \mathrm{~mL})$ and the organic layer was dried over $\mathrm{MgSO}_{4}$. After filtration and evaporation of volatiles, the residue was purified by column chromatography to give the brominated product in $48 \%$ yield, characterized by GC-MS. The product was directly used for the next step.

To a $20 \mathrm{~mL}$ Schelenk tube equipped with magnetic stir bar under nitrogen was added brominated product (631 mg, $2.00 \mathrm{mmol}$ ), and THF $(8 \mathrm{~mL})$. The solution was cooled to $-78{ }^{\circ} \mathrm{C}$ and treated dropwise with ${ }^{n} \mathrm{BuLi}(2.5 \mathrm{M}$ in hexane, $0.96 \mathrm{~mL}, 2.4 \mathrm{mmol})$. After stirring at $-78{ }^{\circ} \mathrm{C}$ for 10 minutes, $\mathrm{D}_{2} \mathrm{O}(0.4 \mathrm{~mL}, 20$ mmol) was slowly added to the reaction mixture. The mixture was stirred for 10 minutes at room temperature and then diluted with $\mathrm{Et}_{2} \mathrm{O}(10 \mathrm{~mL})$ and additional water $(5 \mathrm{~mL})$ and the layers separated. The aqueous layer was extracted with $\mathrm{Et}_{2} \mathrm{O}(10 \mathrm{~mL})$ and the combined organic layers were dried over sodium sulfate, filtered, and concentrated under reduced pressure. The crude material was purified by 
column chromatography to give 2,4-diphenyl-4-methyl-1-[1D]-pentene as a colorless oil (399 $\mathrm{mg}, 84 \%$ yield, 79\% deuteration).

General procedure for deuterium labelling experiments: In an $\mathrm{N}_{2}$-filled dry box, norbornene (37.7 mg, 0.4 mmol), H(D)BPin $(29.3 \mu \mathrm{L}, 0.2 \mathrm{mmol})$, and vinylarene $(0.24 \mathrm{mmol})$ were added to a $4-\mathrm{mL}$ screw-capped vial containing a magnetic stirring bar. $\mathrm{Fe}\left(\mathrm{PMe}_{3}\right)_{4}$ in hexane $(0.4 \mathrm{~mL})$ was added to the mixture. The vial was sealed with a cap containing a PTFE septum and removed from the dry box. The reaction mixture was stirred at $50^{\circ} \mathrm{C}$ for 6 or $18 \mathrm{~h}$ and then cooled to room temperature. The mixture was concentrated in vacuum and the residue was purified by column chromatography on silica gel to give the product.

\section{Detection of norbornane with GC-MS}

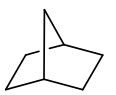

Chemical Formula: $\mathrm{C}_{7} \mathrm{H}_{12}$

Exact Mass: 96.1

For the reaction of $\alpha$-methylstyrene with HBpin:

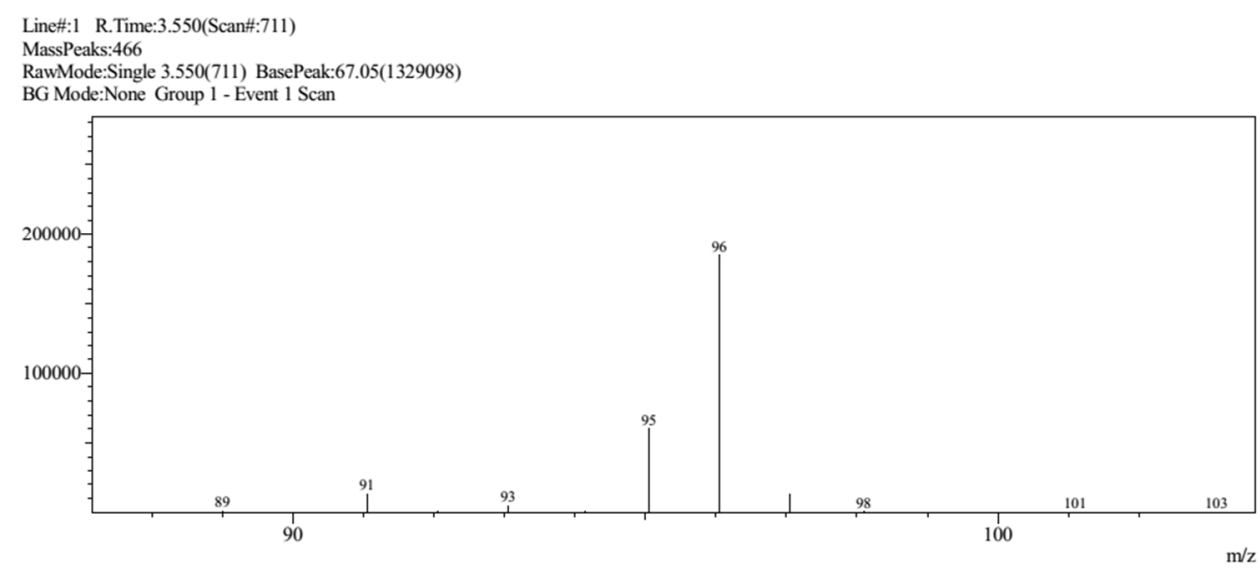

For the reaction of $\alpha$-methylstyrene with DBpin:

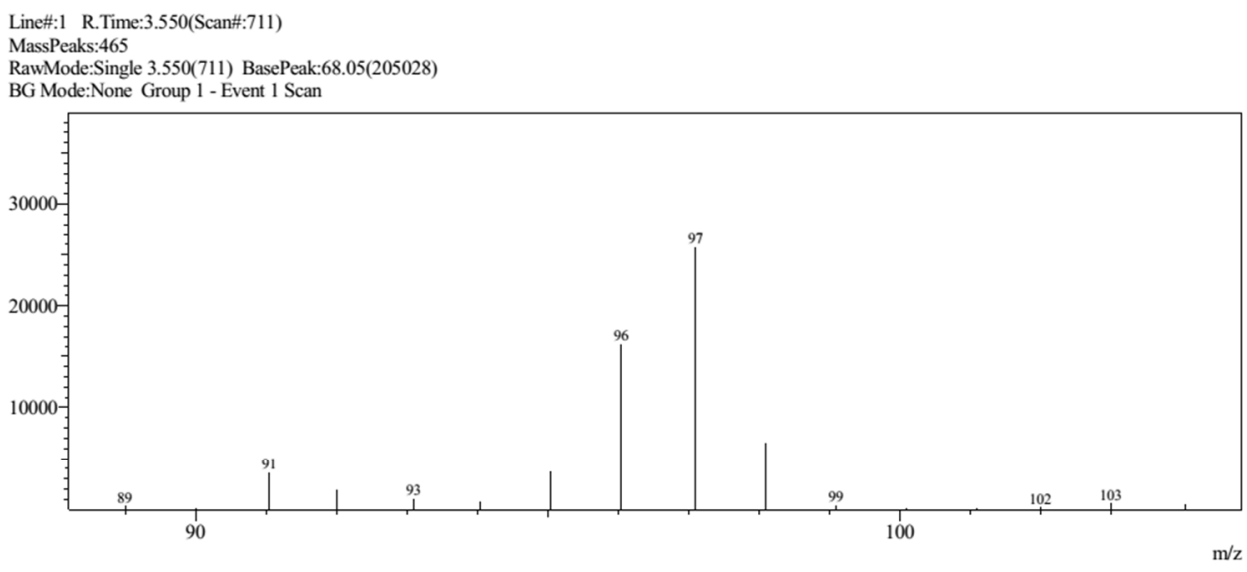

For the reaction of 7-d2 with HBpin: 


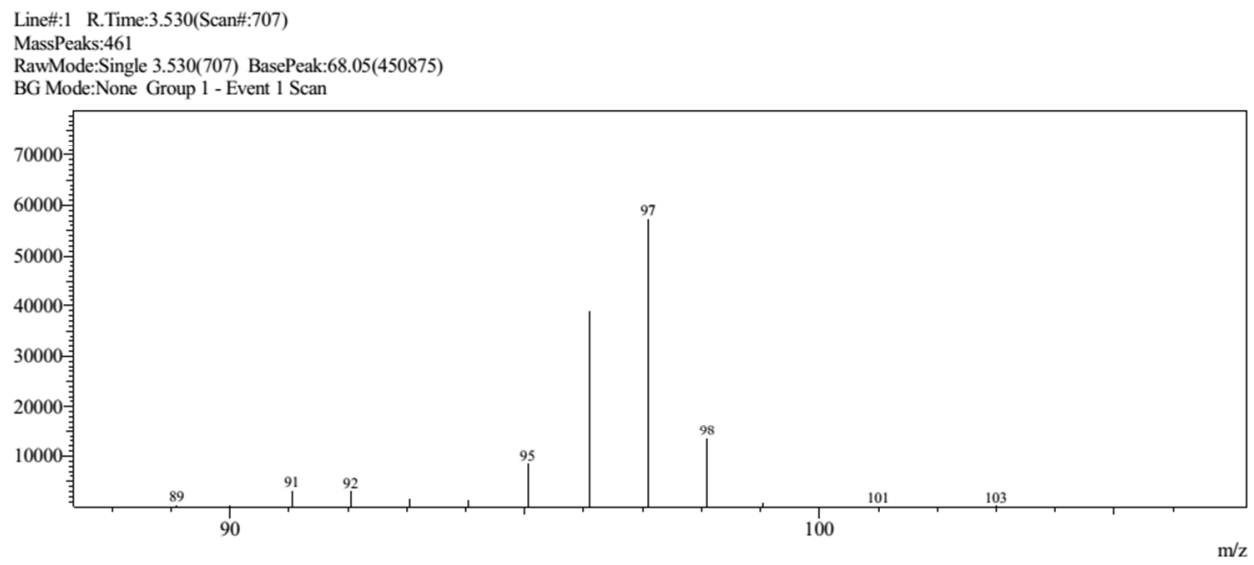

\section{Isomerization Experiment of $\boldsymbol{Z}$-1a under the Standard Reaction Conditions}

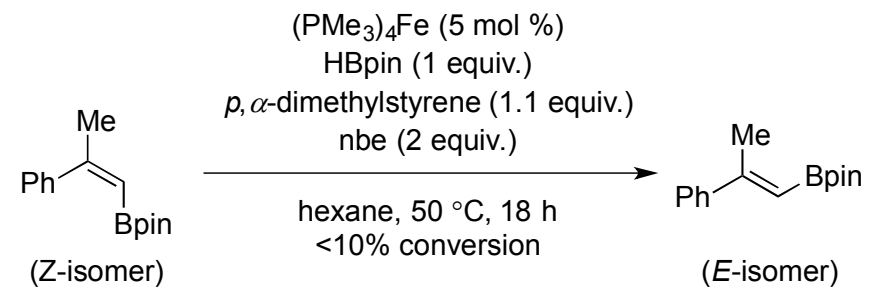

$Z$-1a was prepared according to a previously reported literature. ${ }^{19}$

In an $\mathrm{N}_{2}$-filled dry box, norbornene (37.7 mg, $0.4 \mathrm{mmol}$ ), HBPin (29.3 $\left.\mu \mathrm{L}, 0.2 \mathrm{mmol}\right), p, \alpha$ dimethylstyrene $(29.1 \mathrm{mg}, 0,22 \mathrm{mmol})$, and $\boldsymbol{Z}$-1a $(48.8 \mathrm{mg}, 0,2 \mathrm{mmol})$ were added to a 4 -mL screwcapped vial containing a magnetic stirring bar. $\mathrm{Fe}\left(\mathrm{PMe}_{3}\right)_{4}(3.6 \mathrm{mg}, 0.01 \mathrm{~mol})$ in hexane $(0.4 \mathrm{~mL})$ was added to the mixture. The vial was sealed with a cap containing a PTFE septum and removed from the dry box. The reaction mixture was stirred at $50{ }^{\circ} \mathrm{C}$ for $18 \mathrm{~h}$ and the conversion was determined by GC.

\section{Competition Experiment between Vinylarene and $\beta, \beta$-2D-Vinylarene}

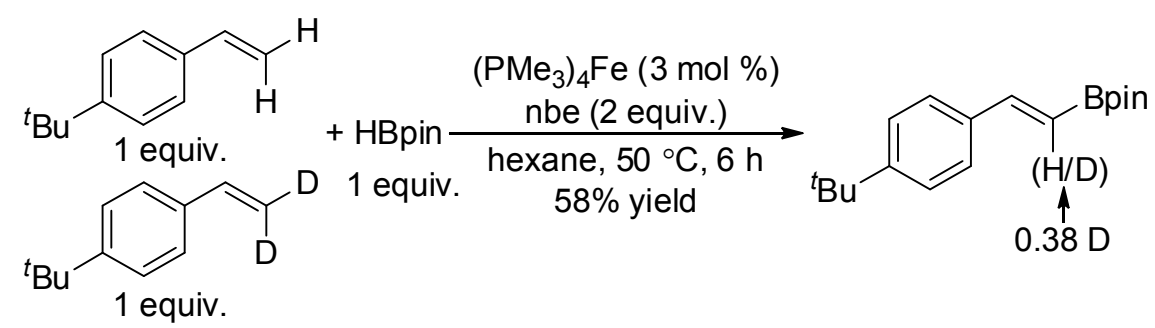

In an $\mathrm{N}_{2}$-filled dry box, norbornene (37.7 mg, $\left.0.4 \mathrm{mmol}\right)$, HBPin $(29.3 \mu \mathrm{L}, 0.2 \mathrm{mmol})$, 4-tert-butylstyrene (32.1 $\mathrm{mg}, 0,2 \mathrm{mmol}$ ), and $\beta, \beta$-2D-4-tert-butylstyrene $(32.5 \mathrm{mg}, 0,2 \mathrm{mmol})$ were added to a 4-mL screwcapped vial containing a magnetic stirring bar. $\mathrm{Fe}\left(\mathrm{PMe}_{3}\right)_{4}(2.2 \mathrm{mg}, 0.006 \mathrm{~mol})$ in hexane $(0.4 \mathrm{~mL})$ was added to the mixture. The vial was sealed with a cap containing a PTFE septum and removed from the dry box. The reaction mixture was stirred at $50{ }^{\circ} \mathrm{C}$ for $6 \mathrm{~h}$ and then cooled to room temperature. The mixture was concentrated in vacuum and the residue was purified by column chromatography on silica gel to give the product. The result indicated that the ratio of the products derived from 4-tert-butylstyrene and $\beta, \beta$ 2D-4-tert-butylstyrene was approximately 1.6:1 (The deviation from 1.6 could be possible due to the H/D exchange through the reversible olefin insertion into the $\mathrm{Fe}-\mathrm{H} / \mathrm{D}$ bond). 
Discussion on dehydrogenative borylation of aliphatic alkenes: The dehydrogenative borylation of aliphatic alkenes did not occur under the conditions described in Table 2. However, when conducted under modified reaction conditions with additional 4,4'-di-tert-butyl-2,2'-bipyridyl ligand, the reaction of 1-octene and HBpin afforded the hydroboration product and the dehydrogenative borylation product in $49 \%$ and $27 \%$ GC yield, respectively.

\section{References}

(1) Balaraman, E.; Gnanaprakasam, B.; Shimon, L. J.; Milstein, W. D. J. Am. Chem. Soc. 2010, 132, 16756-16758.

(2) Zhang, L.; Peng, D.; Leng, X.; Huang, Z. Angew. Chem. Int. Ed. 2013, 52, 3676-3680.

(3) Girolami, G. S.; Wilkinson, G. J. Chem. Soc. Dalton Trans. 1985, 1339-1348.

(4) Karsch, H. H. Chem. Ber. 1977, 110, 2222-2235.

(5) Smith, C. R.; Zhang, A.; Mans, D. J.; RajanBabu, T. V. Org. Synth. 2008, 85, 248.

(6) Yilmaz, M. Tetrahedron 2011, 67, 8255-8263.

(7) Bhattacharya, P.; Krause, J. A.; Guan, H. Organometallics 2011, 30, 4720-4729.

(8) (a) Karsch, H. H.; Klein, H.-F.; Schmidbaur, H. Angew. Chem.; Int. Ed. 1974, 14, 637-638. (b) Karsch, H. H.; Klein, H.-F.; Schmidbaur, H. Chem. Ber. 1977, 110, 2200-2212.

(9) Alfaro, R.; Parra, A.; Aleman, J.; Ruano, J. L. G.; Tortosa, M. J. Am. Chem. Soc. 2012, 134, 1516515168.

(10) Wang, C.; Xu, Z.; Tobrman, T.; Negishi, E. Adv. Synth. Catal. 2010, 352, 627-631.

(11) Coapes, R. B.; Souza, F. E. S.; Thomas, R. L.; Halla, J. J.; Marder, Todd B. Chem. Commun. 2003, 614-615.

(12) Murata, M.; Watanabe, S.; Masuda, Y. Tetrahedron. Letters. 1999, 40, 2585-2588.

(13) Reid, W. B.; Spillane, J. J.; Krause, S. B.; Watson, D. A. J. Am. Chem. Soc. 2016, 138, 5539-5542.

(14) Ho, H. E.; Asao, N.; Yamamoto, Y.; Jin, T. Org. Lett. 2014, 16, 4670-4673.

(15) Yang, Z.; Zhong, M.; Ma, X.; Nijesh, K.; De, S.; Parameswaran, P.; Roesky, H. W. J. Am. Chem.

Soc. 2016, 138, 2548-2551.

(16) Zhao, Y.-W.; Feng, Q.; Song, Q.-L. Chinese. Chemical. Letters. 2016, 27, 571-574.

(17) Labre, F.; Gimbert, Y.; Bannwarth, P.; Olivero, S.; Duñach, E.; Chavant, P. Y. Org. Lett. 2014, 16, 2366-2369.

(18) Lipshutz, B. H.; Butler, T.; Lower, A. J. Am. Chem. Soc. 2006, 128, 15396-15398.

(19) Hu, T.-J.; Zhang, G.; Chen, Y.-H.; Feng, C.-G.; Lin, G.-Q. J. Am. Chem. Soc. 2016, 138, 2897-2900. 


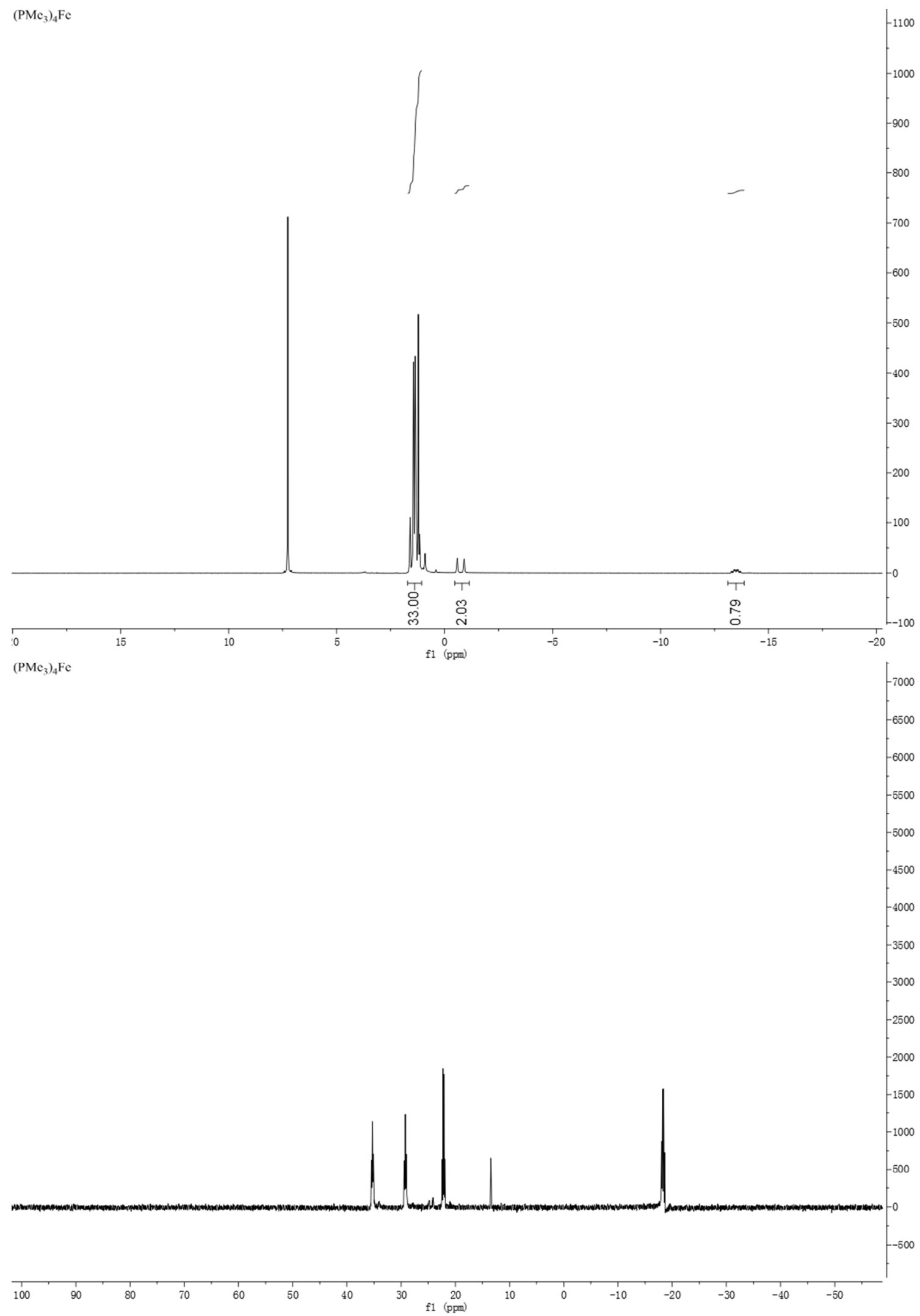


Wang, Wu, and GE, Supporting Information

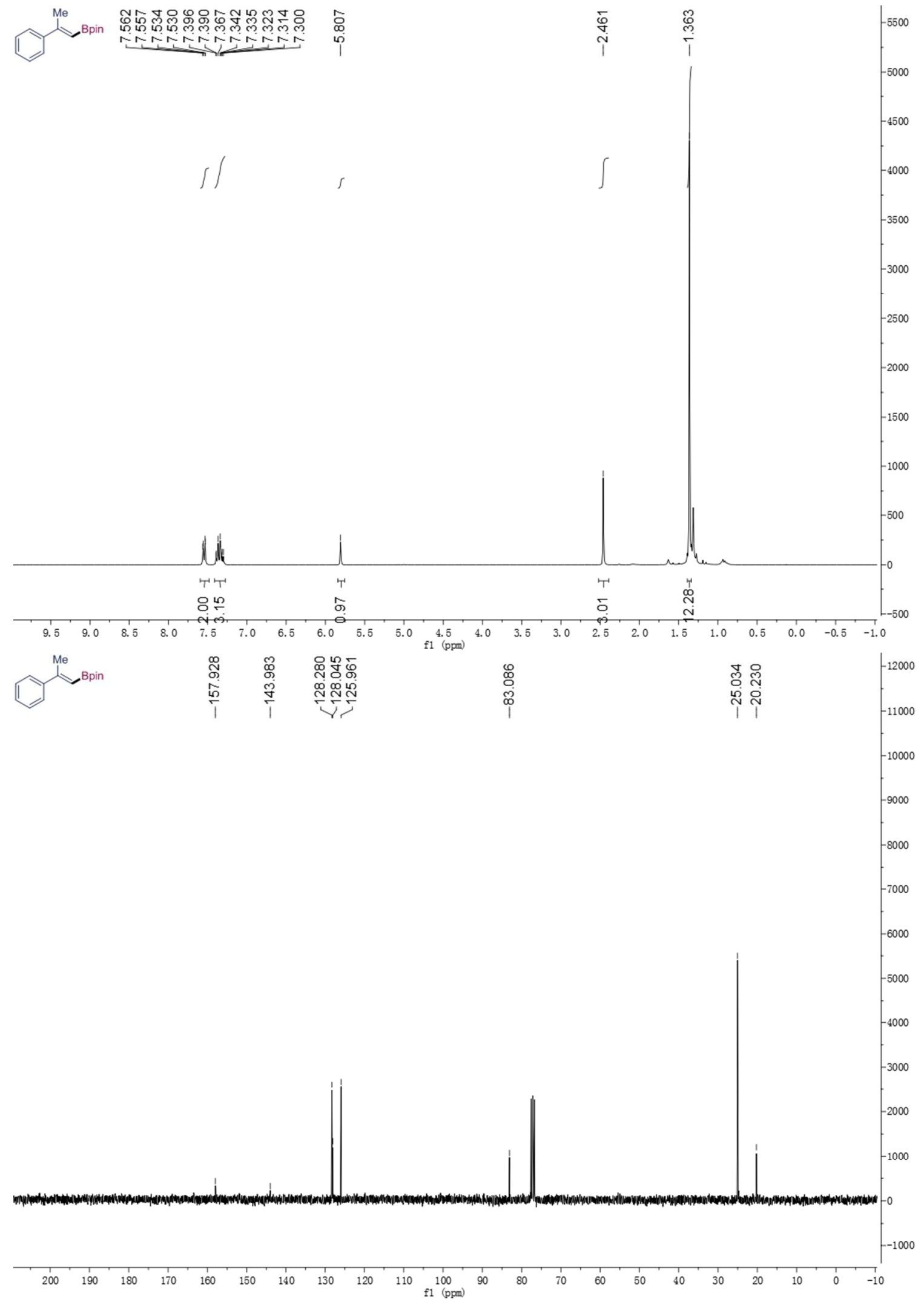


Wang, Wu, and GE, Supporting Information

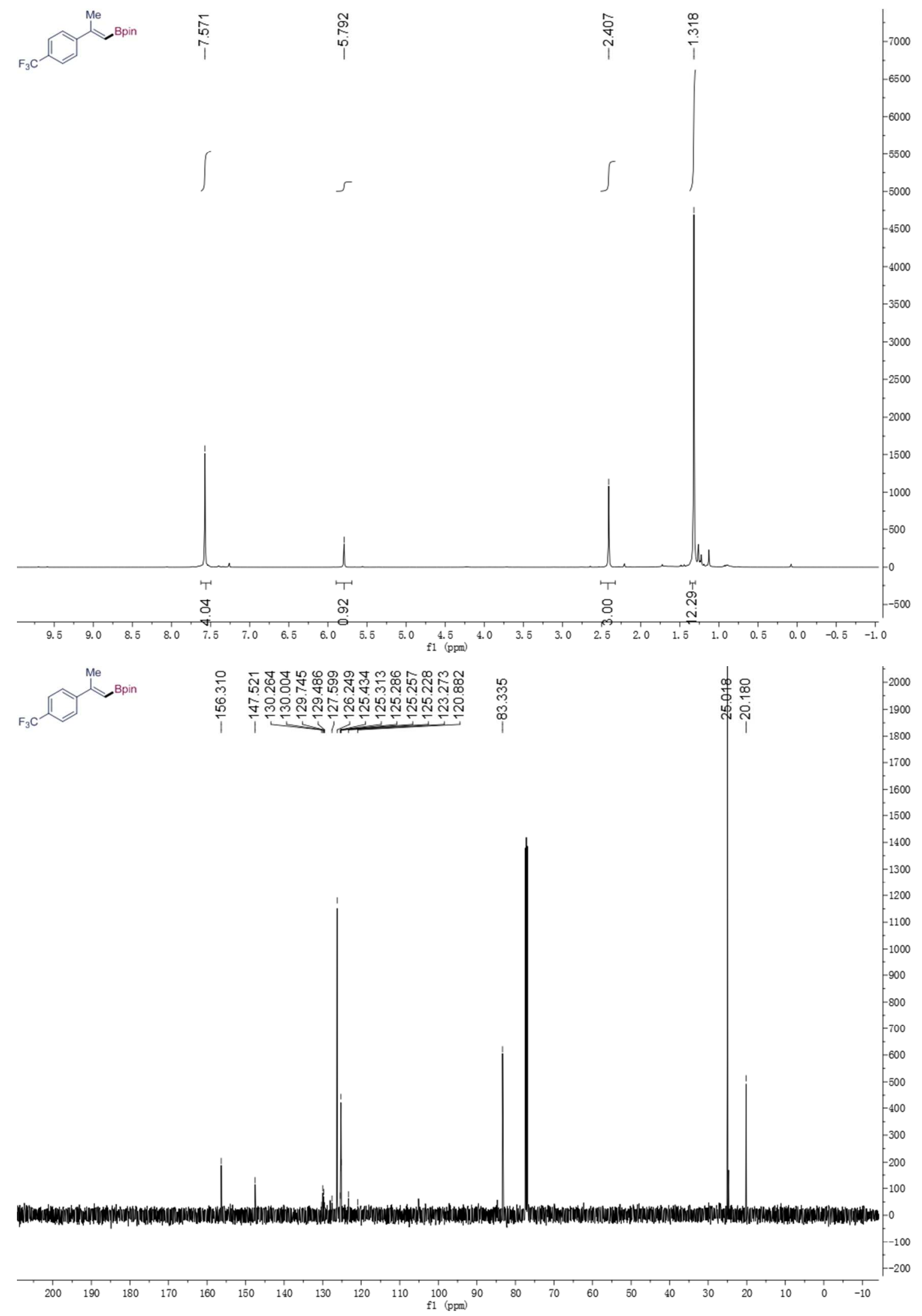


Wang, Wu, and GE, Supporting Information

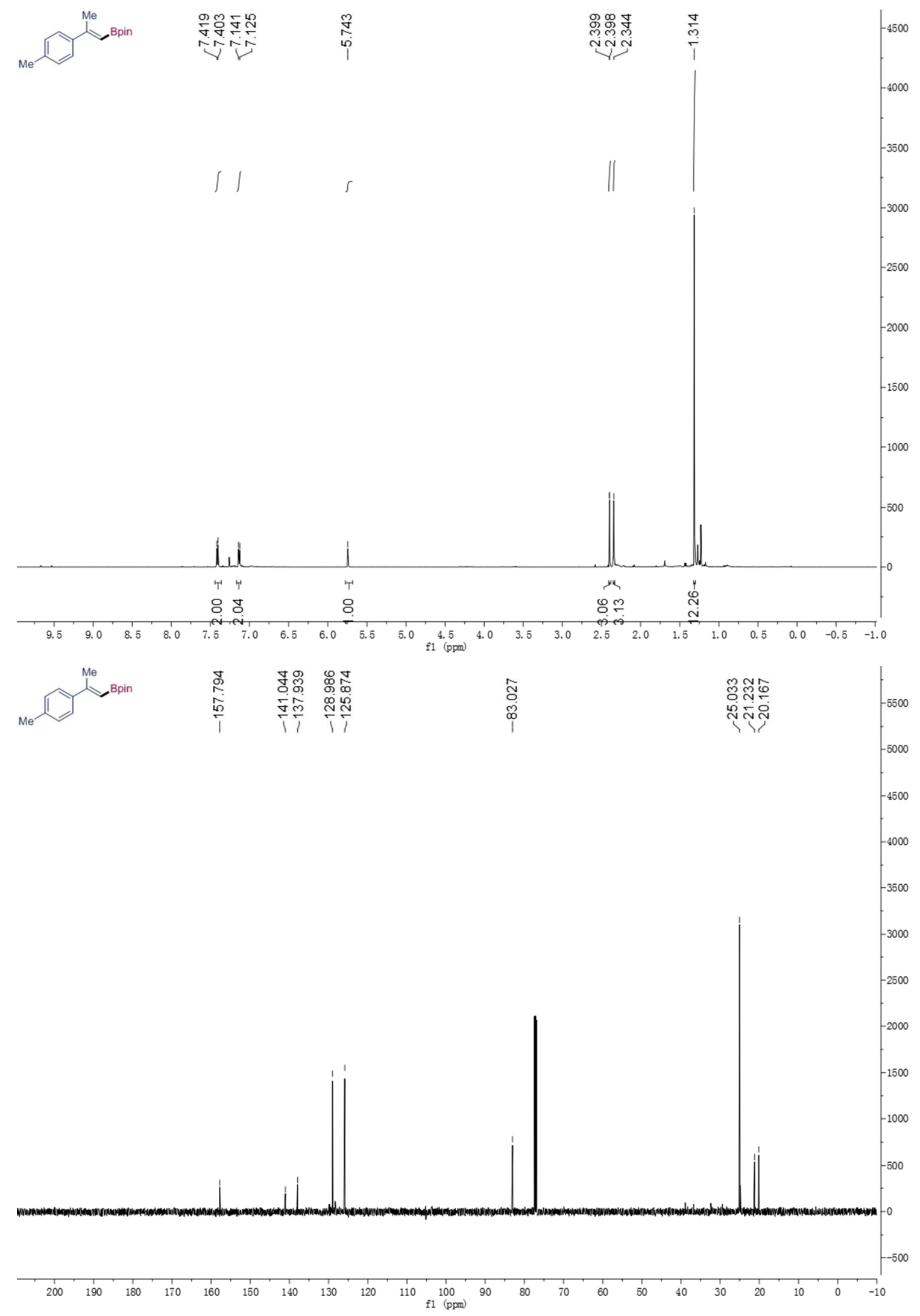


Wang, Wu, and GE, Supporting Information

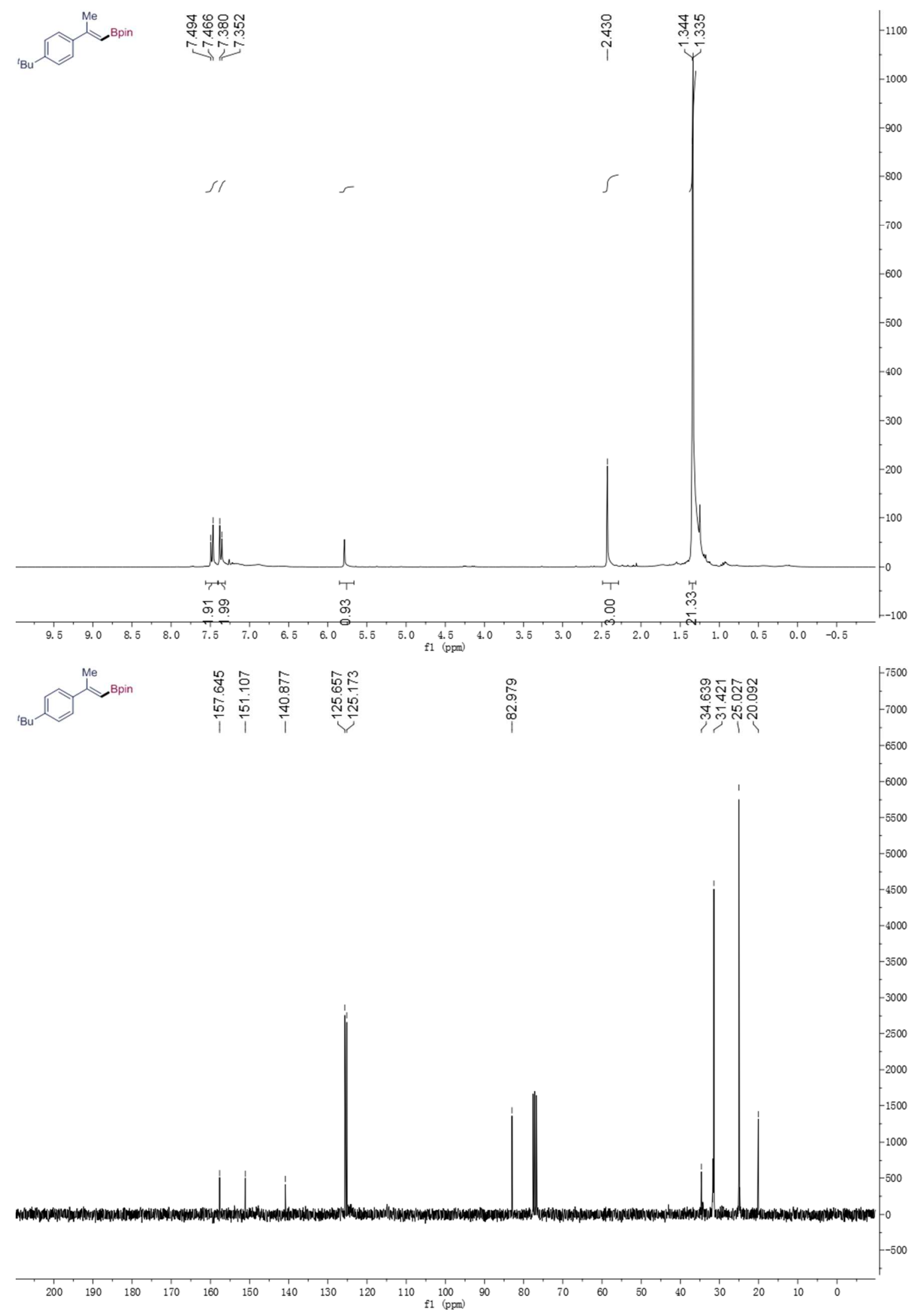


Wang, Wu, and GE, Supporting Information
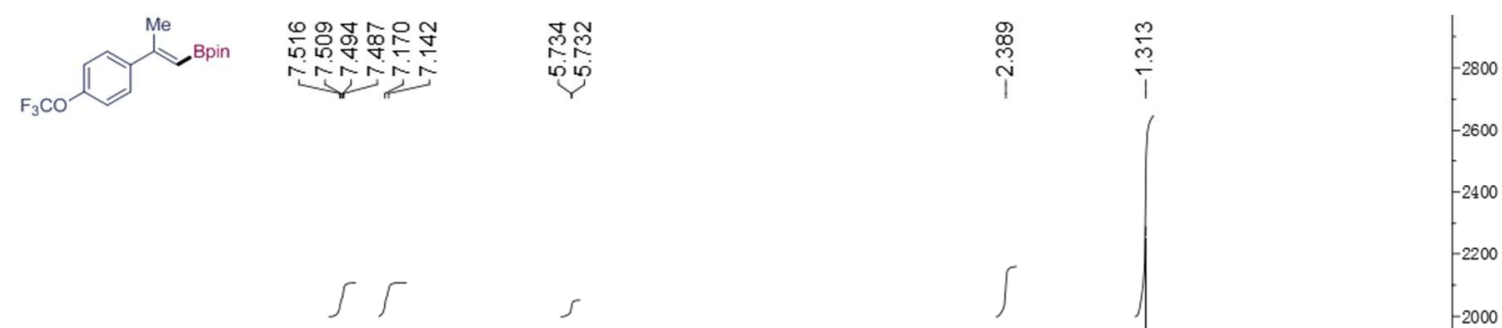

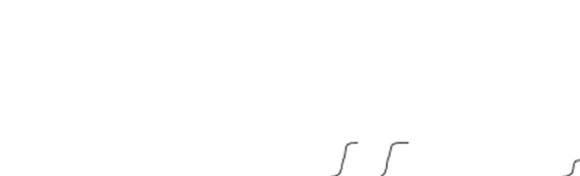

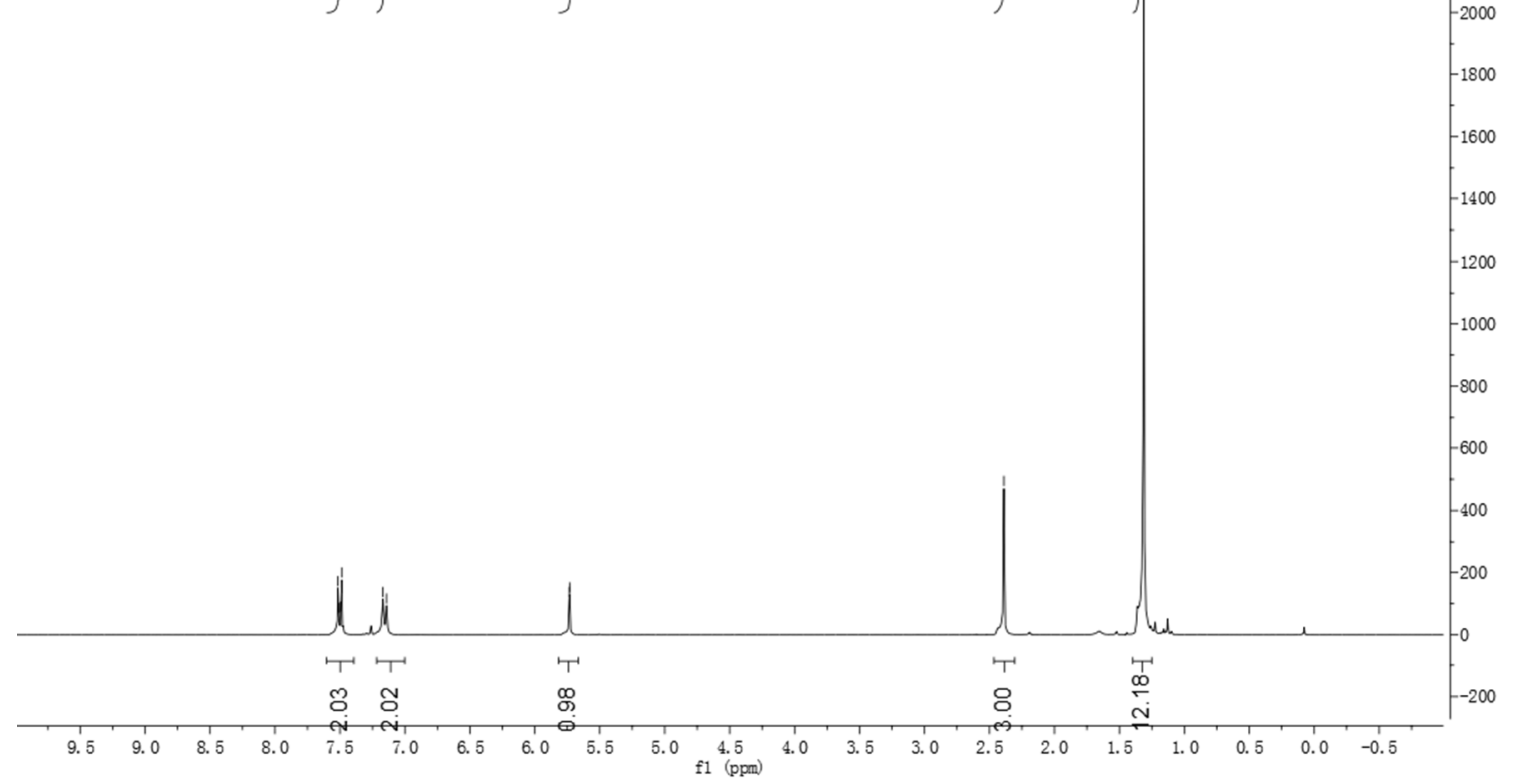

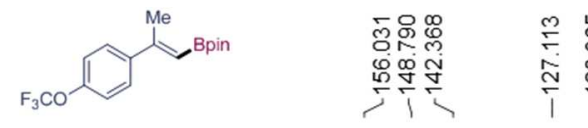

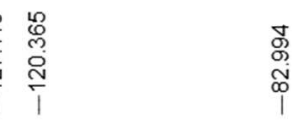

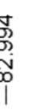

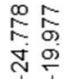

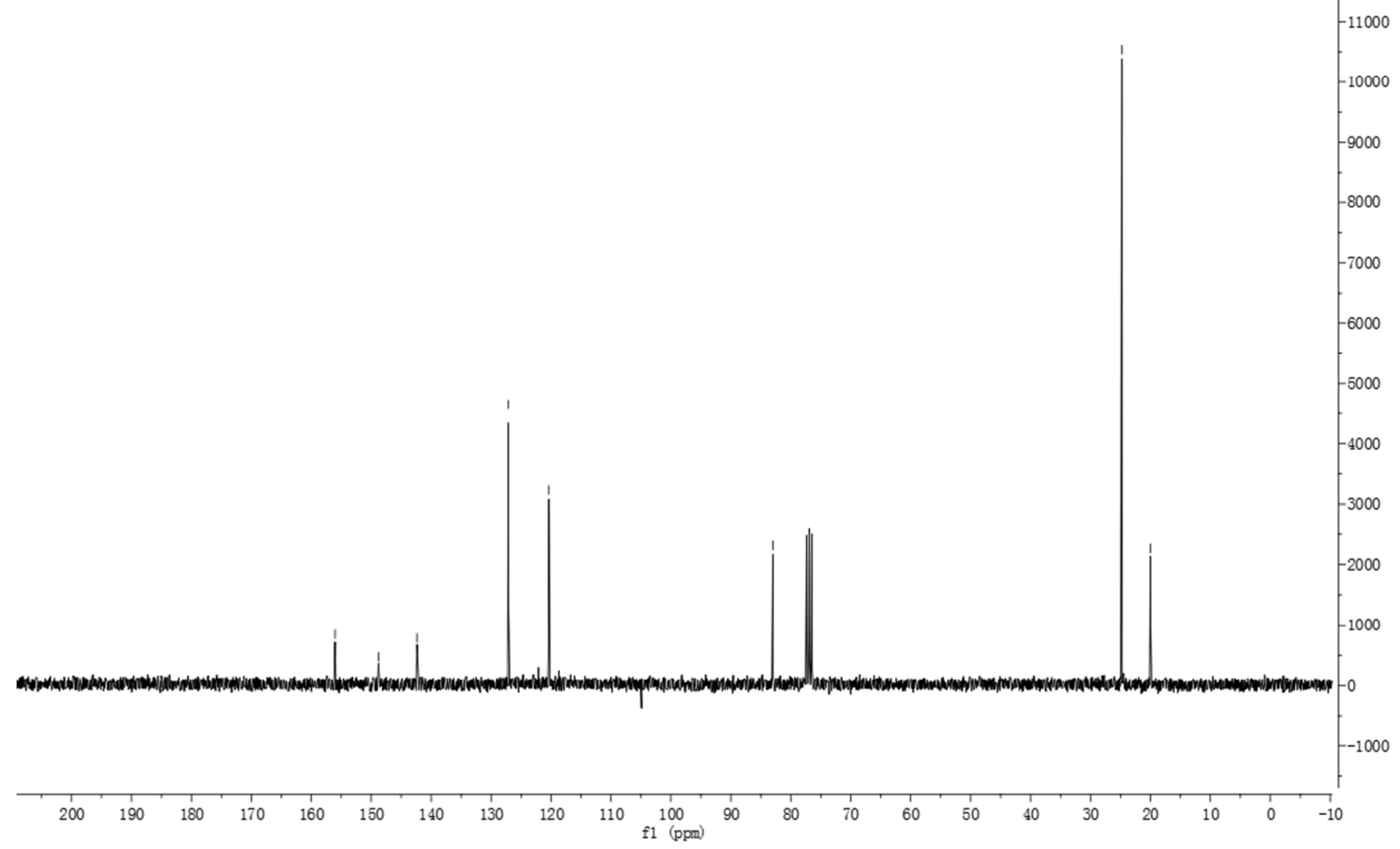


Wang, Wu, and GE, Supporting Information

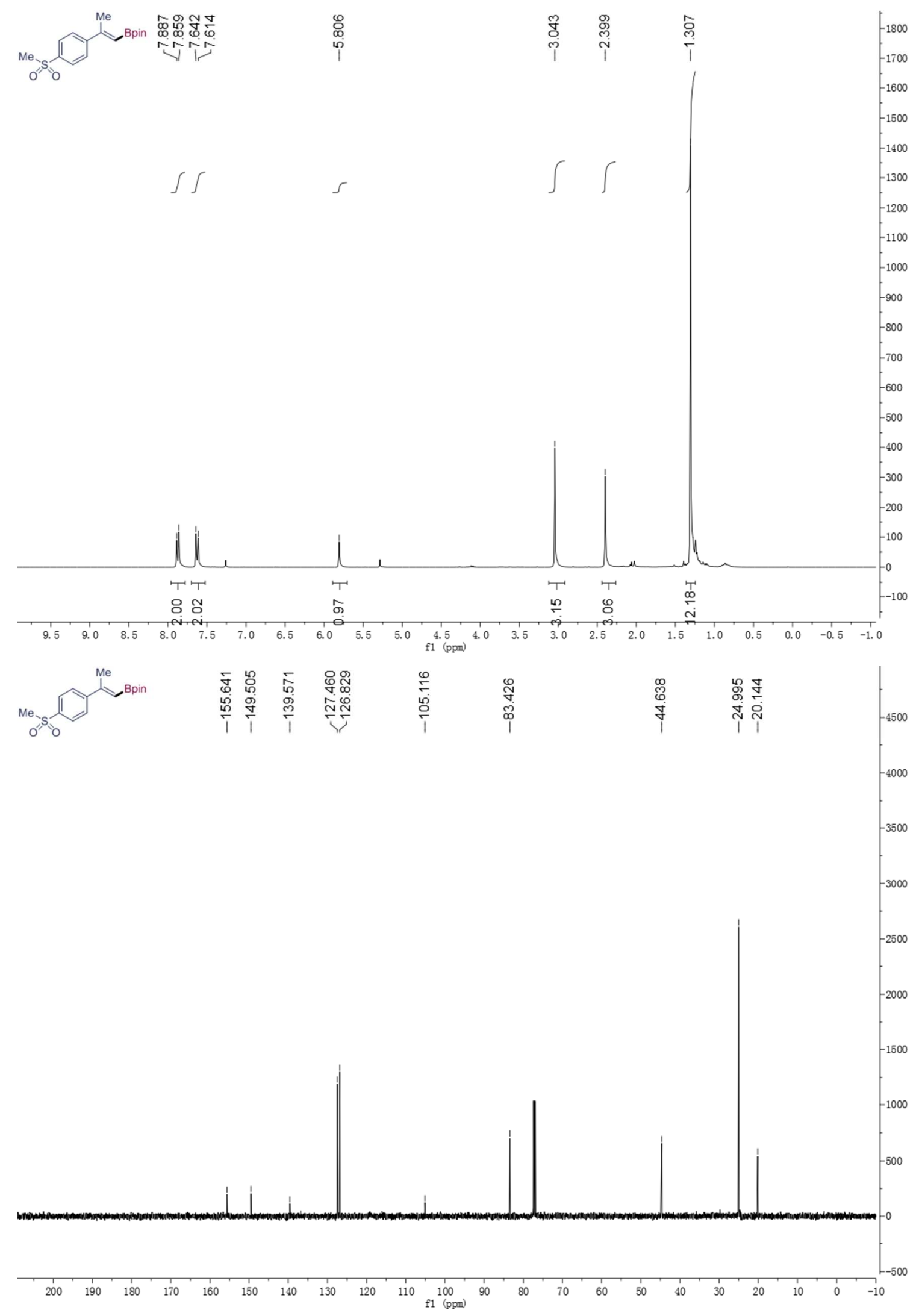




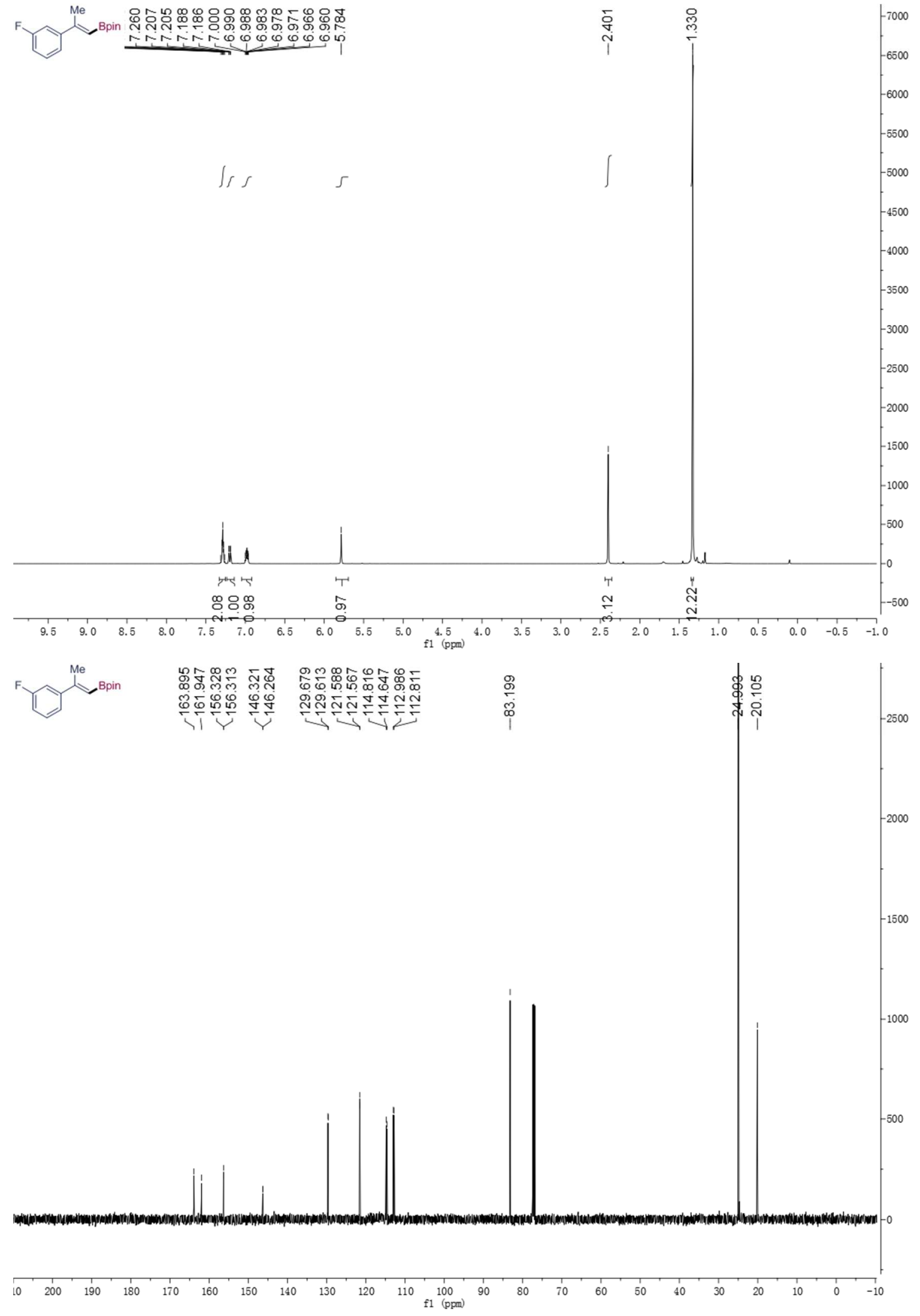




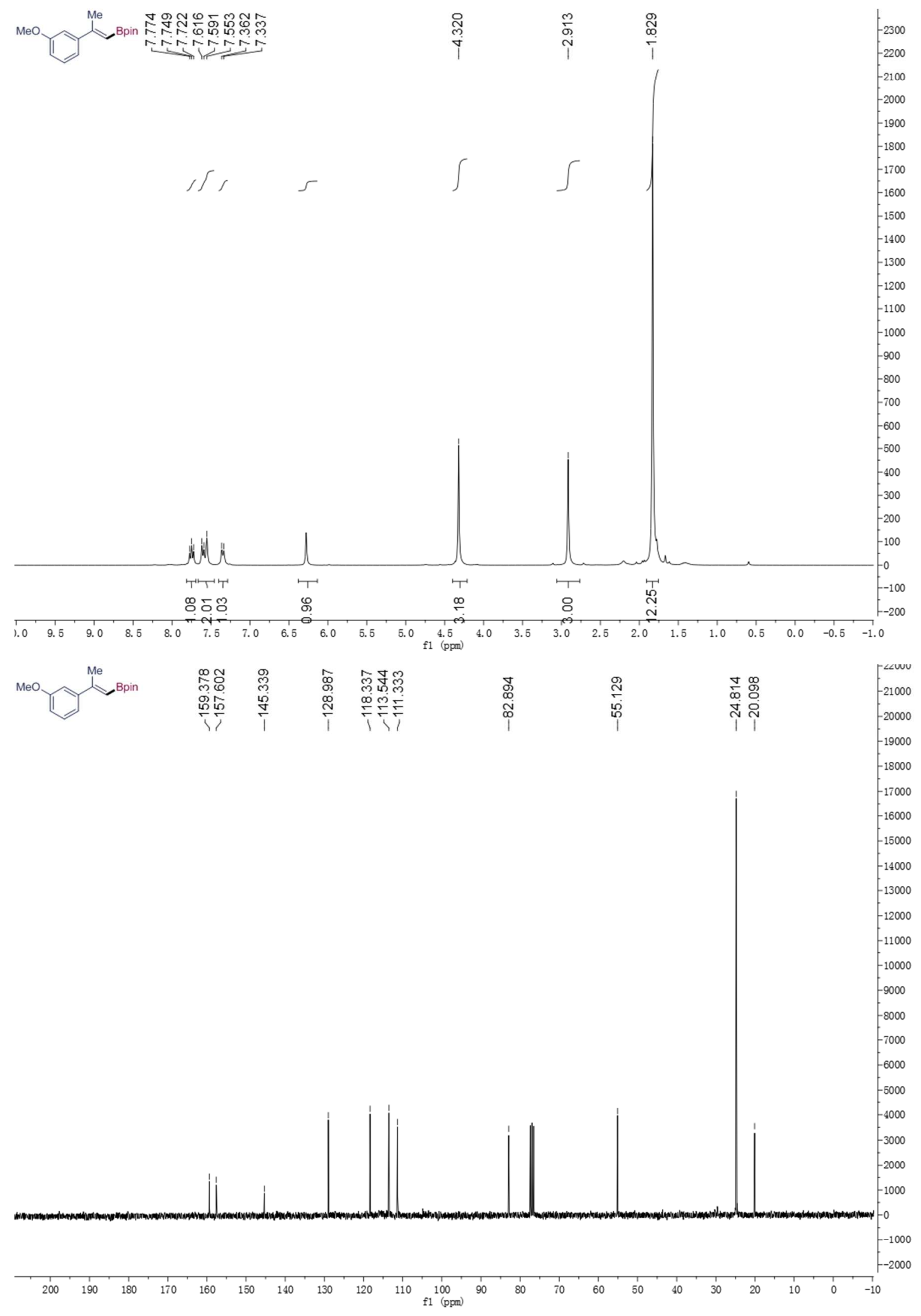


Wang, Wu, and GE, Supporting Information

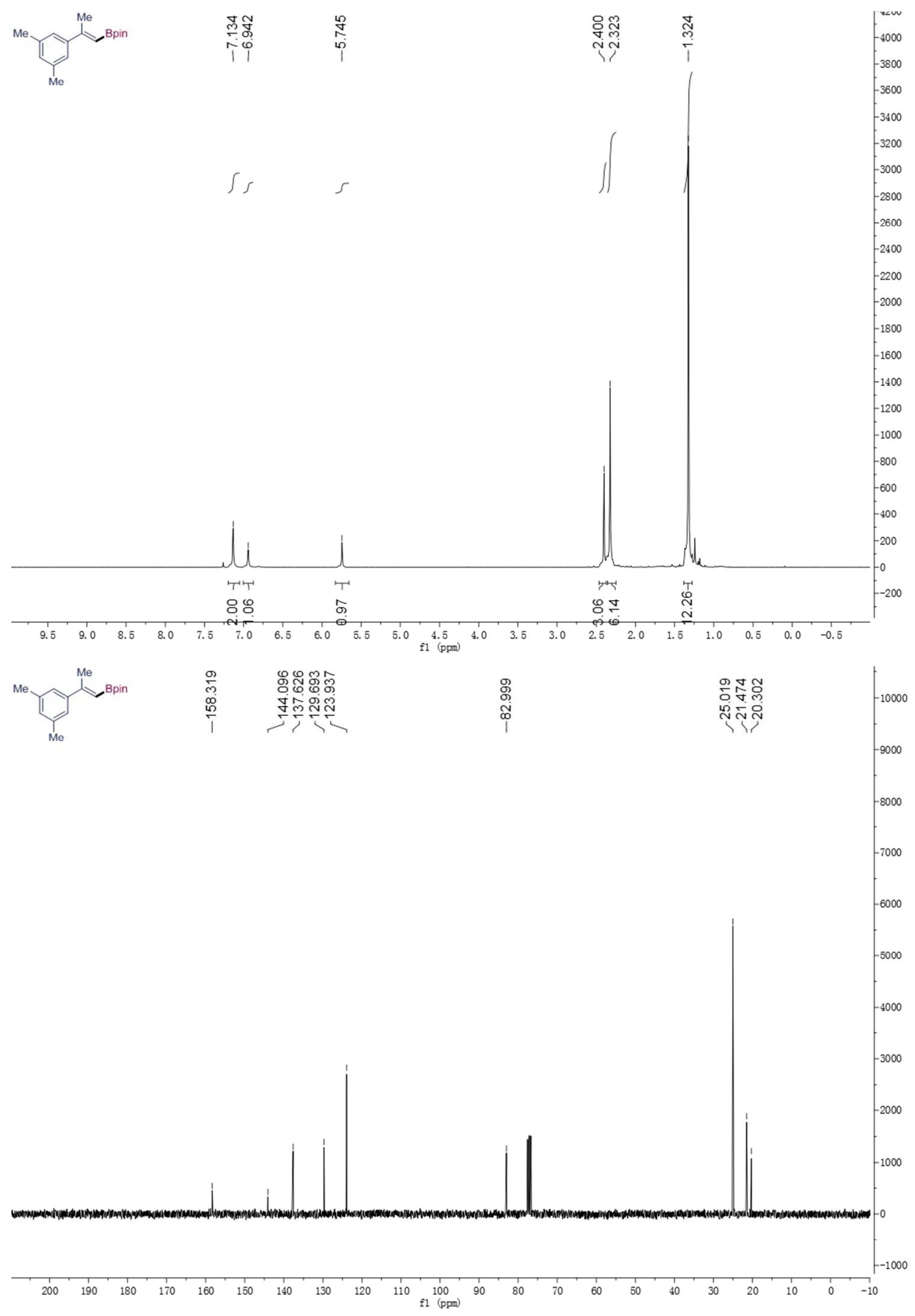




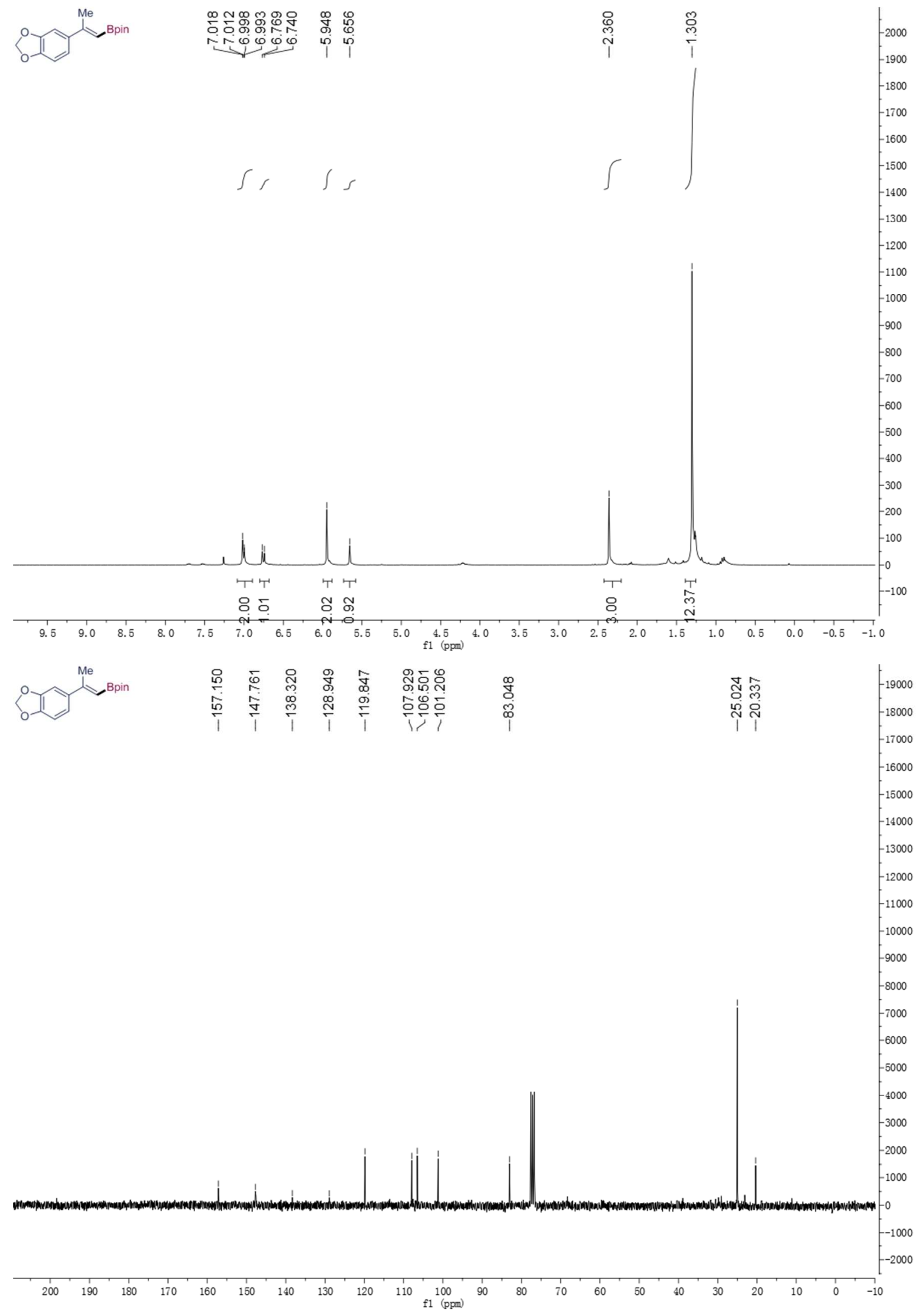


Wang, Wu, and GE, Supporting Information

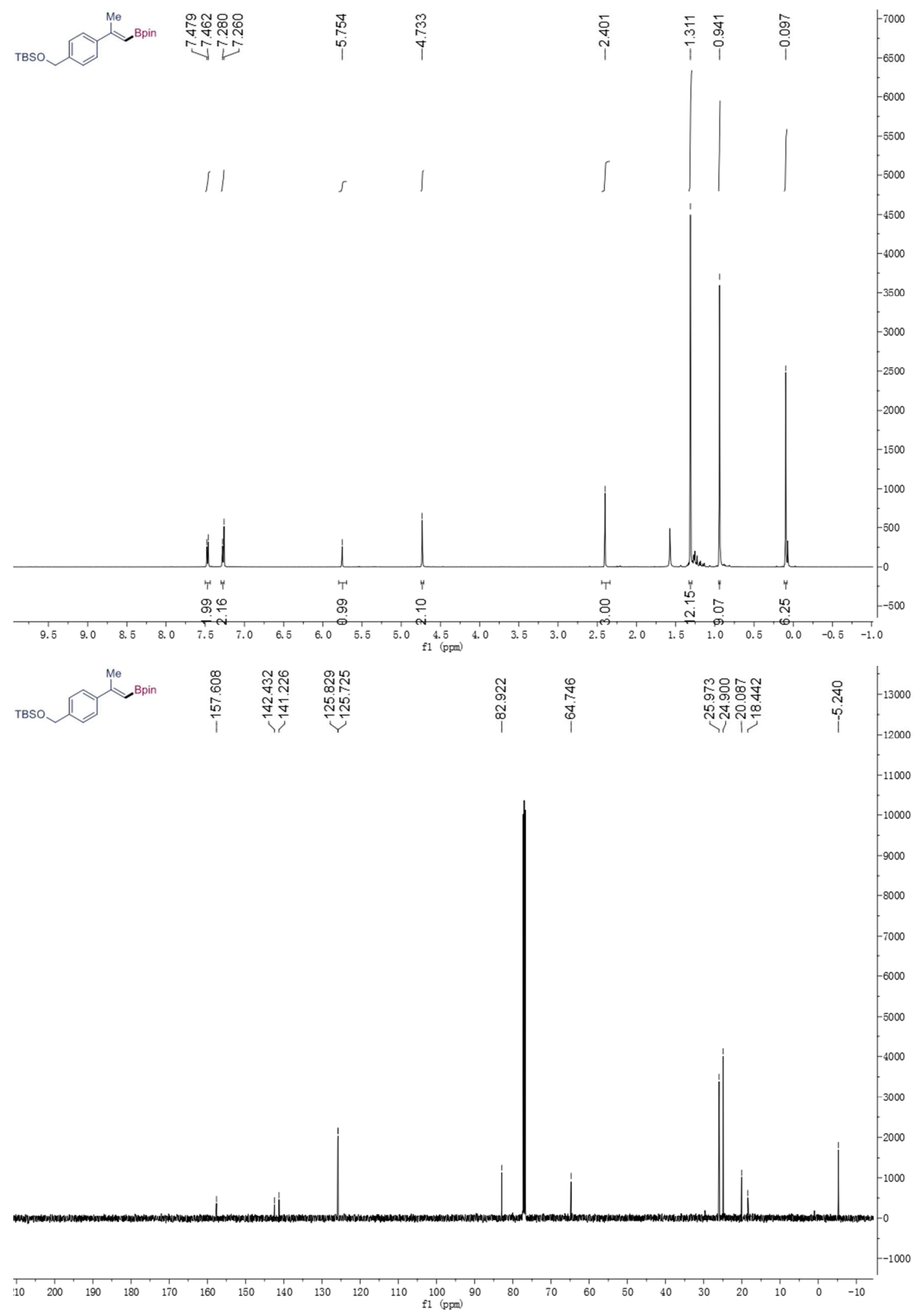


Wang, Wu, and GE, Supporting Information

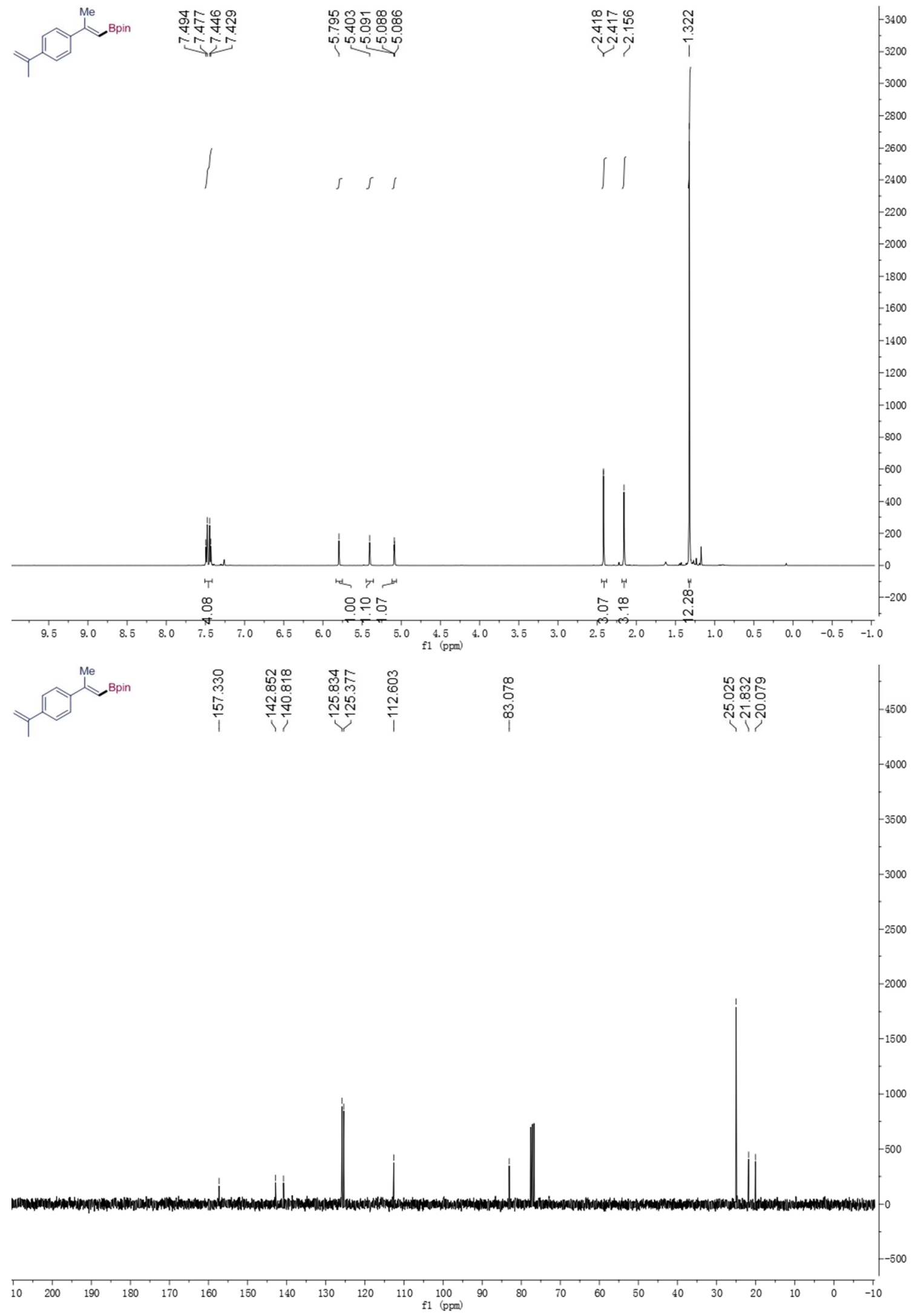




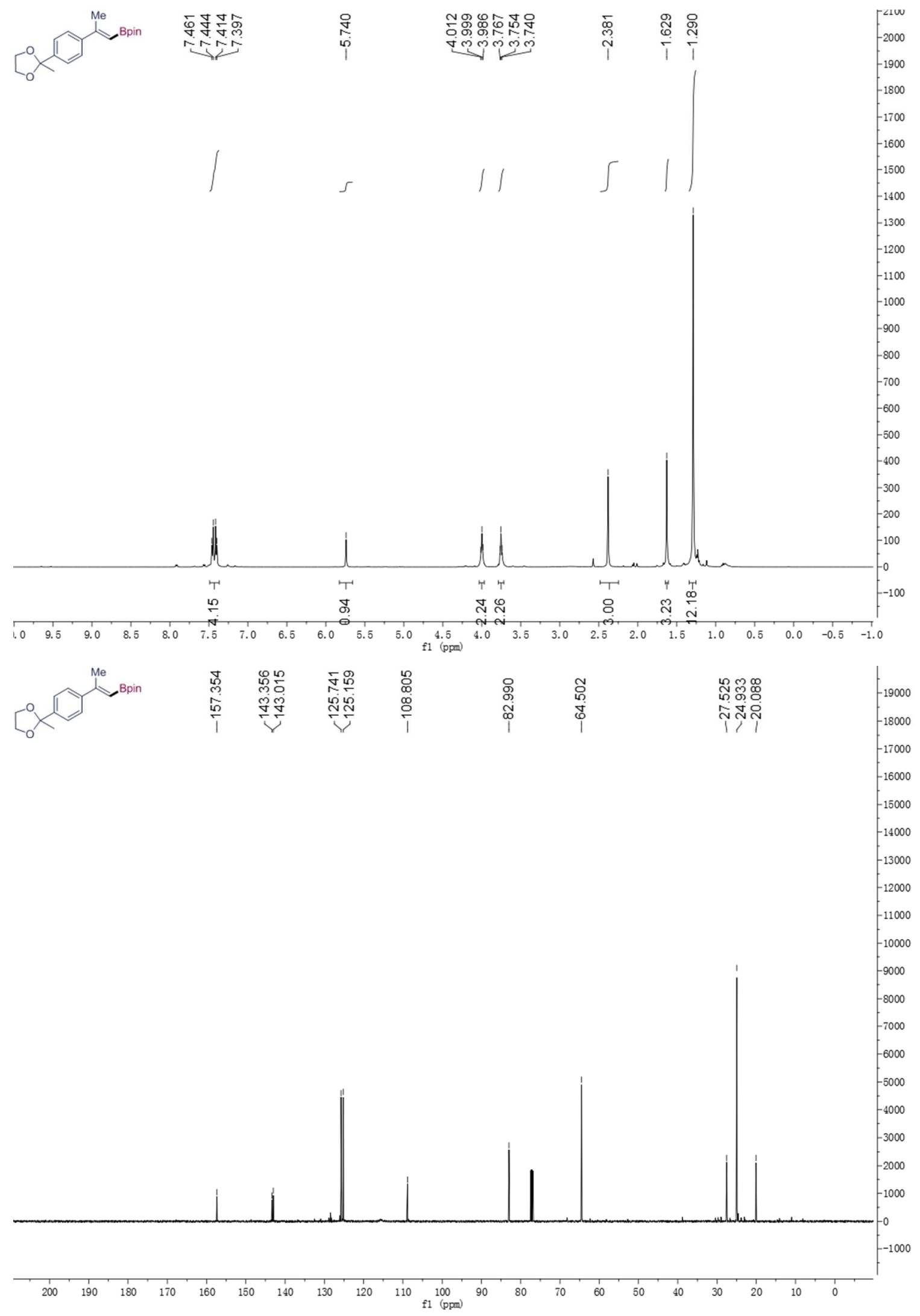




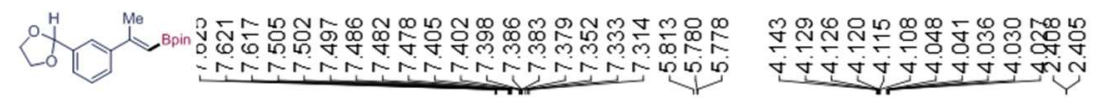

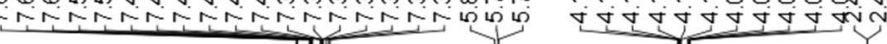

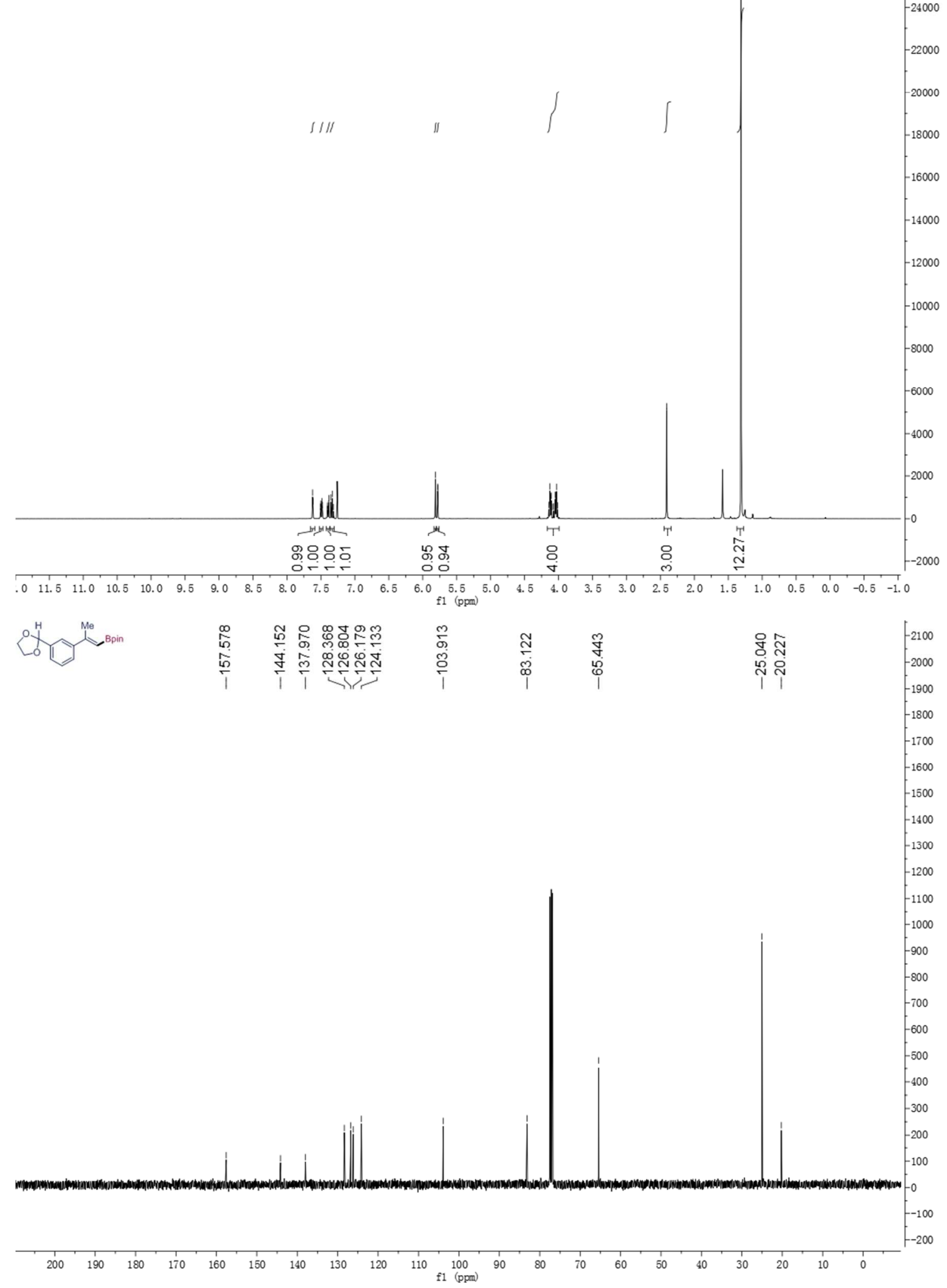




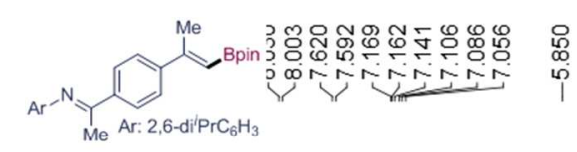

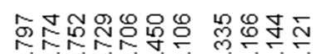

Ninnininis
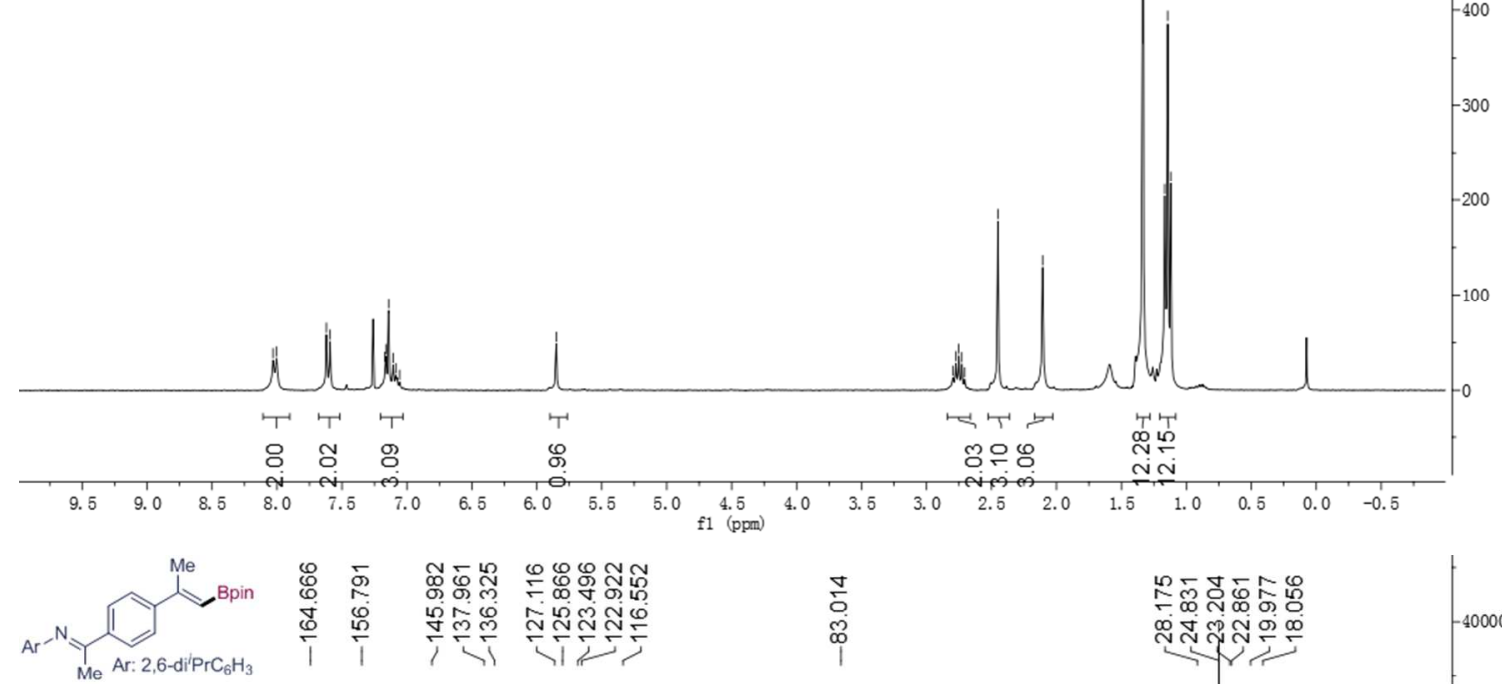

$\square$
$\vdots$

1

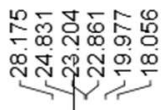

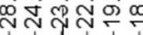

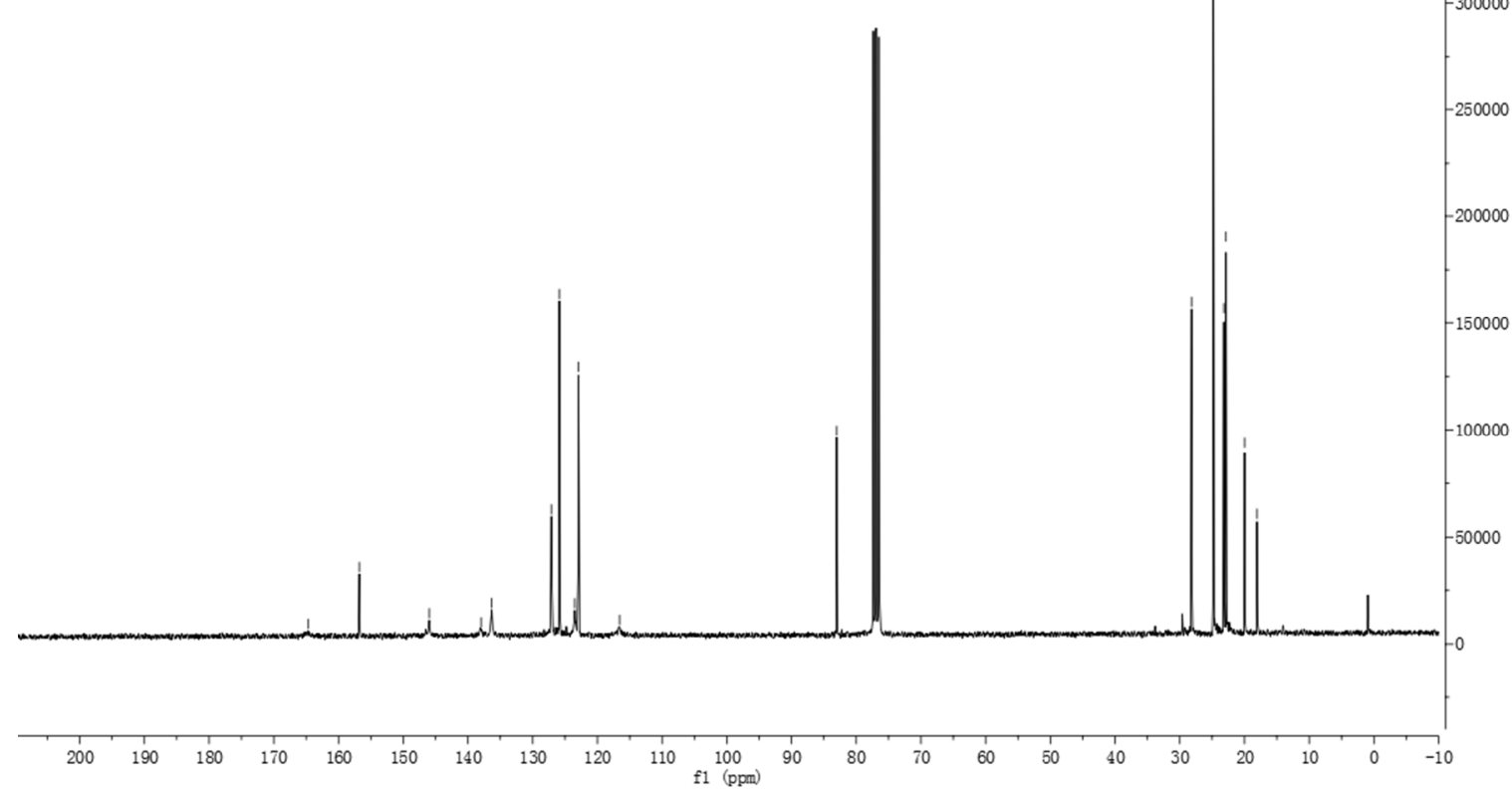


Wang, Wu, and GE, Supporting Information

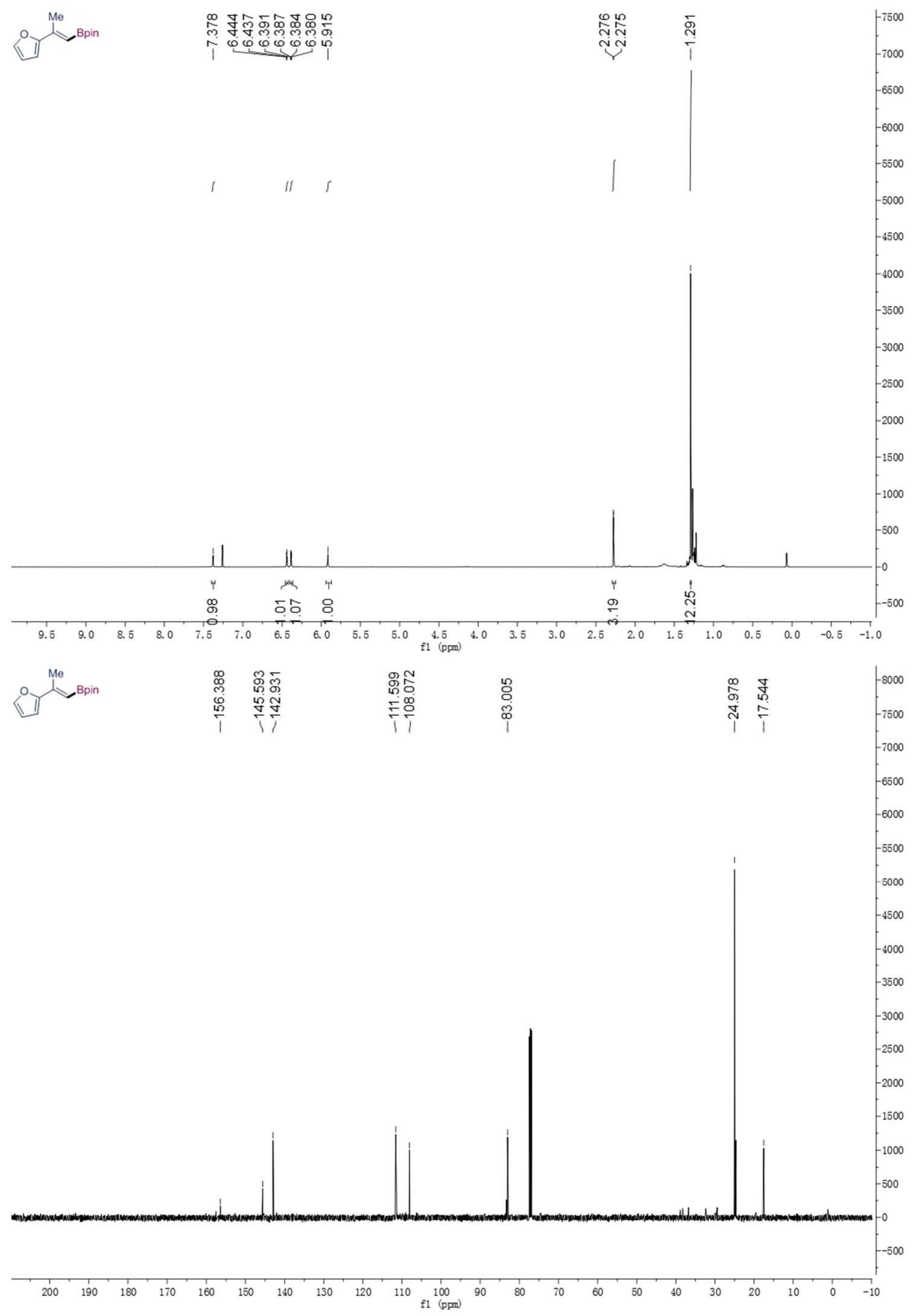




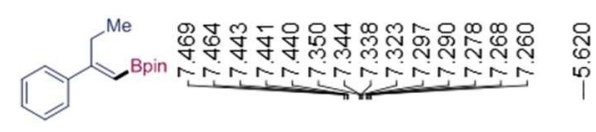

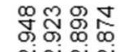

نูin

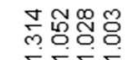

mº

5000

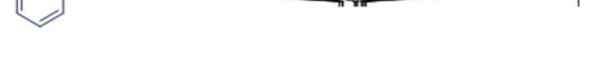

(1)

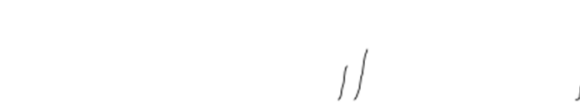

s

/

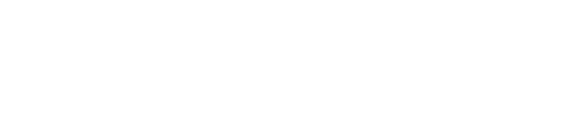

(1)

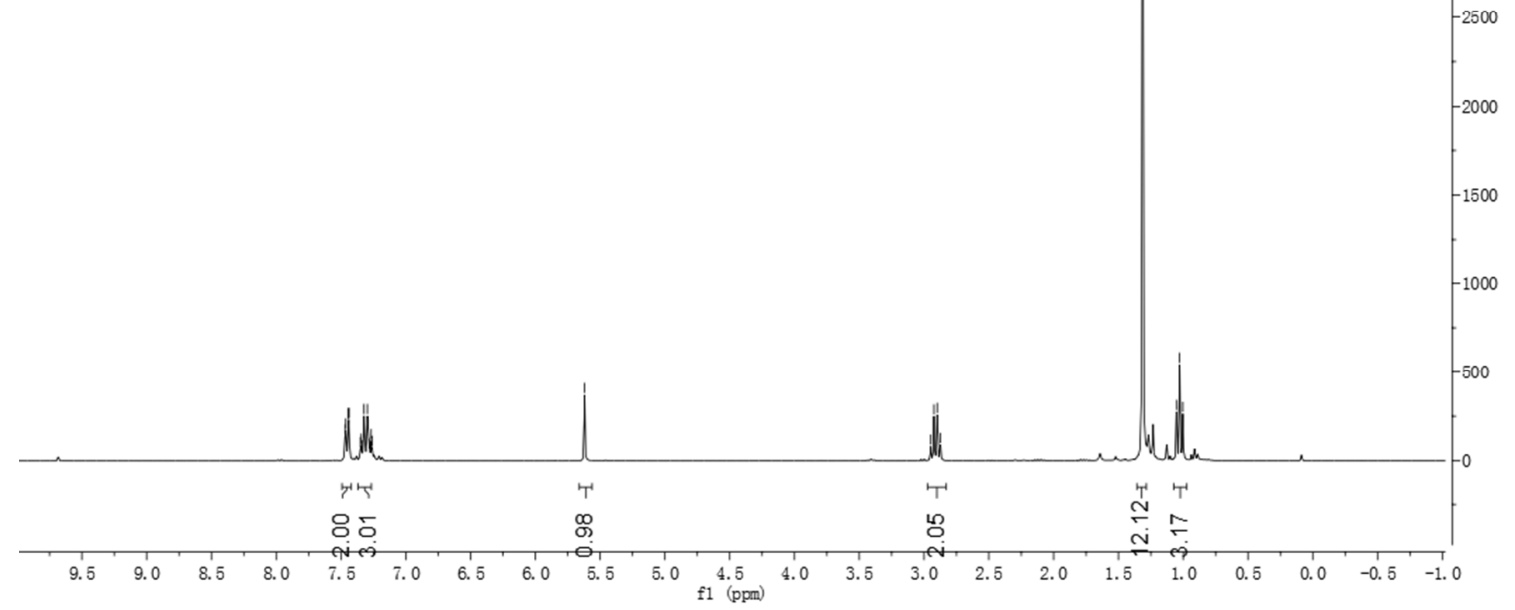

$\infty$
$\infty$
$\sim$
$\infty$
1

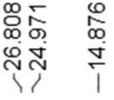

$-10000$
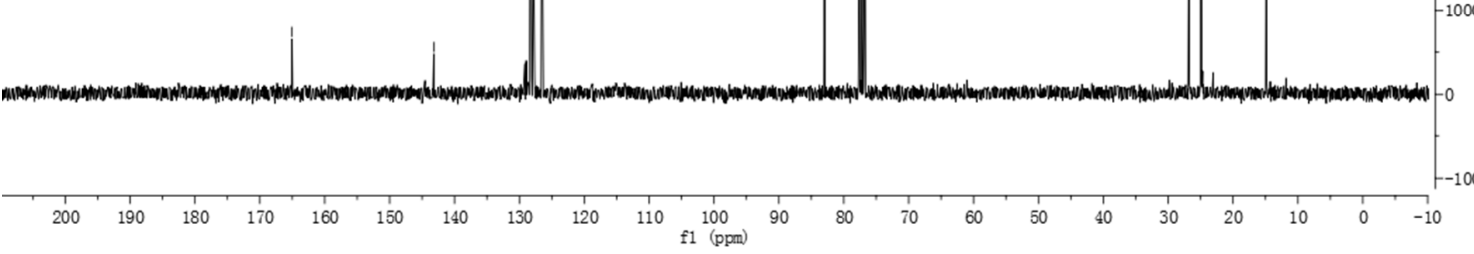


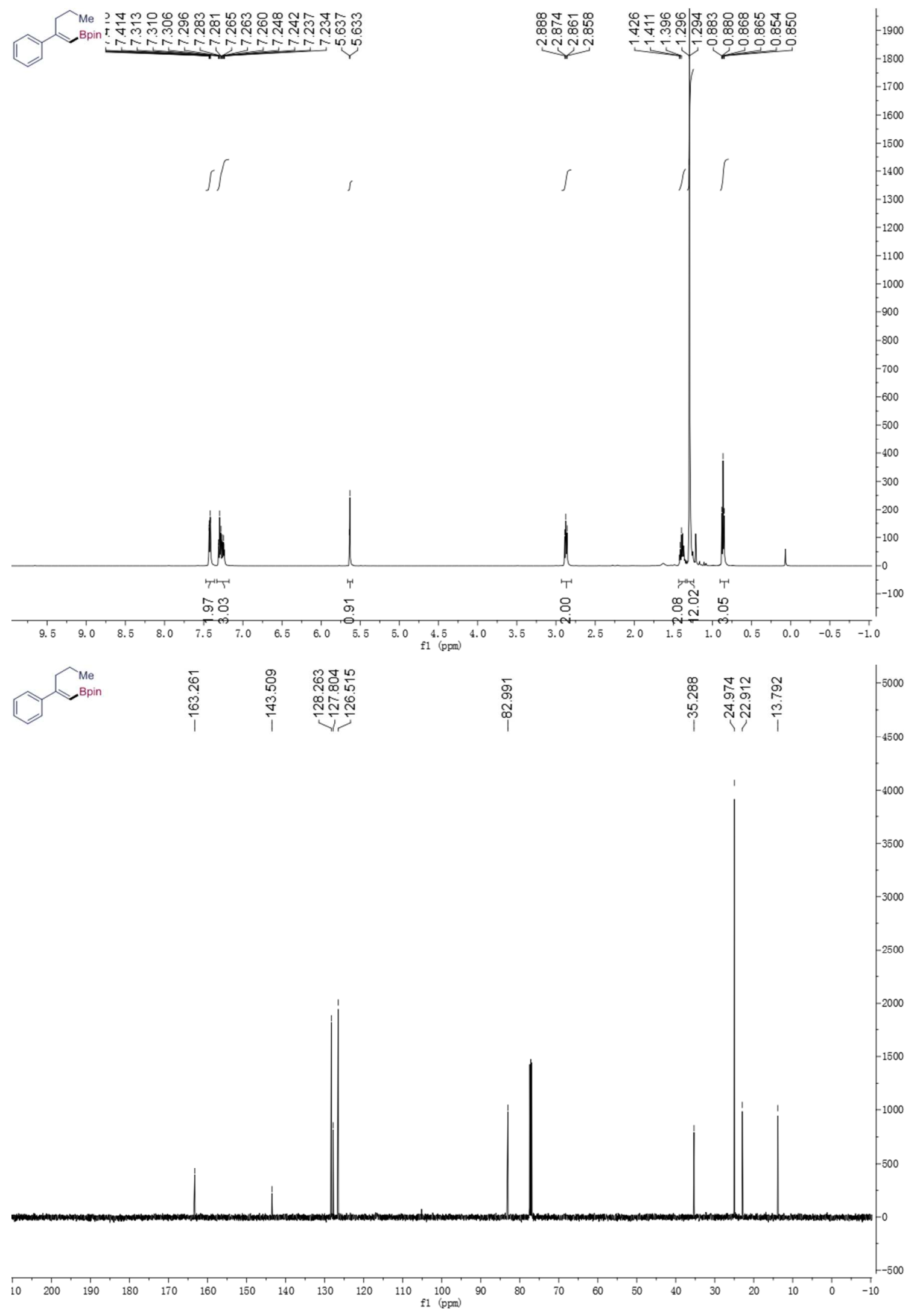




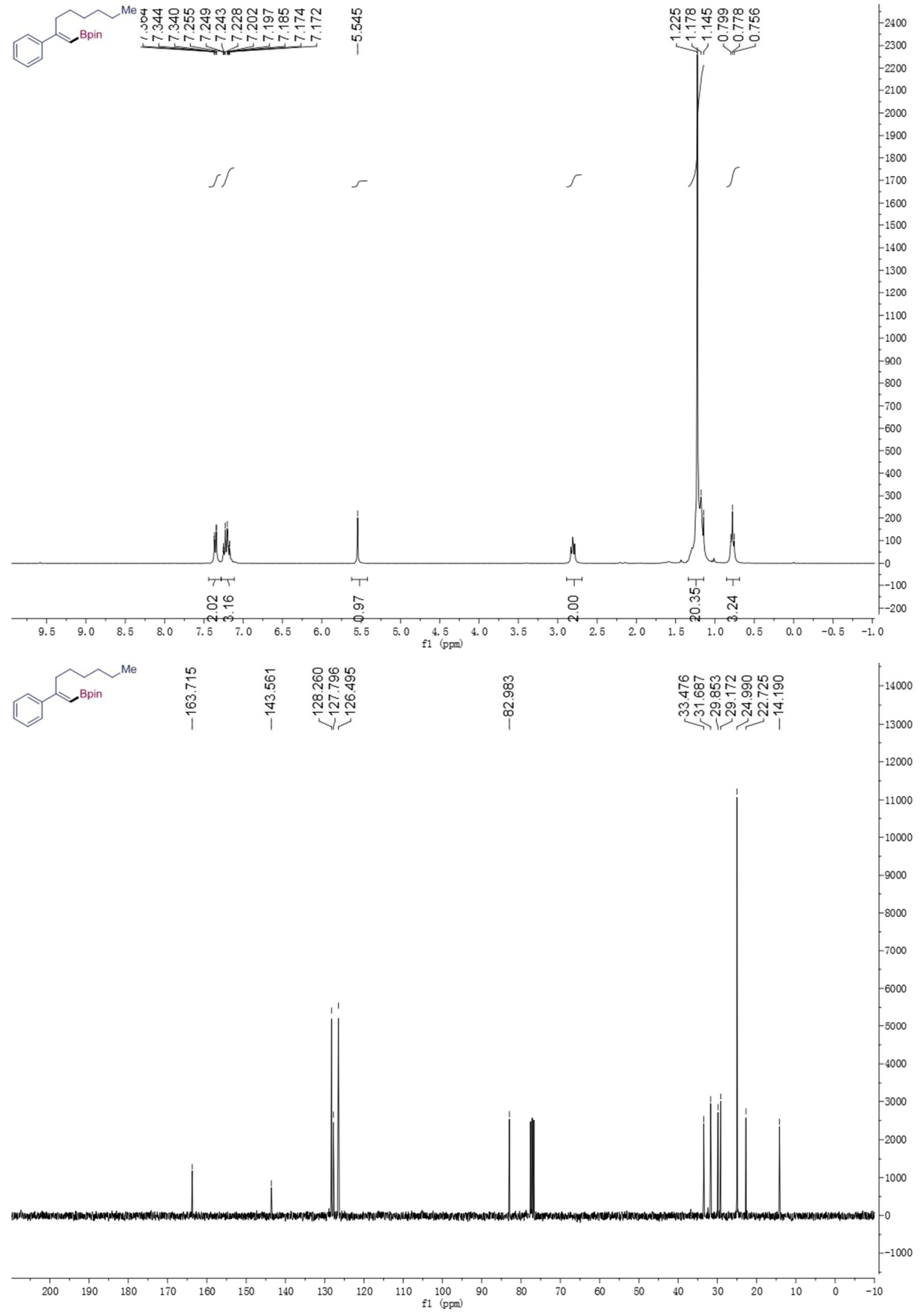


Wang, Wu, and GE, Supporting Information
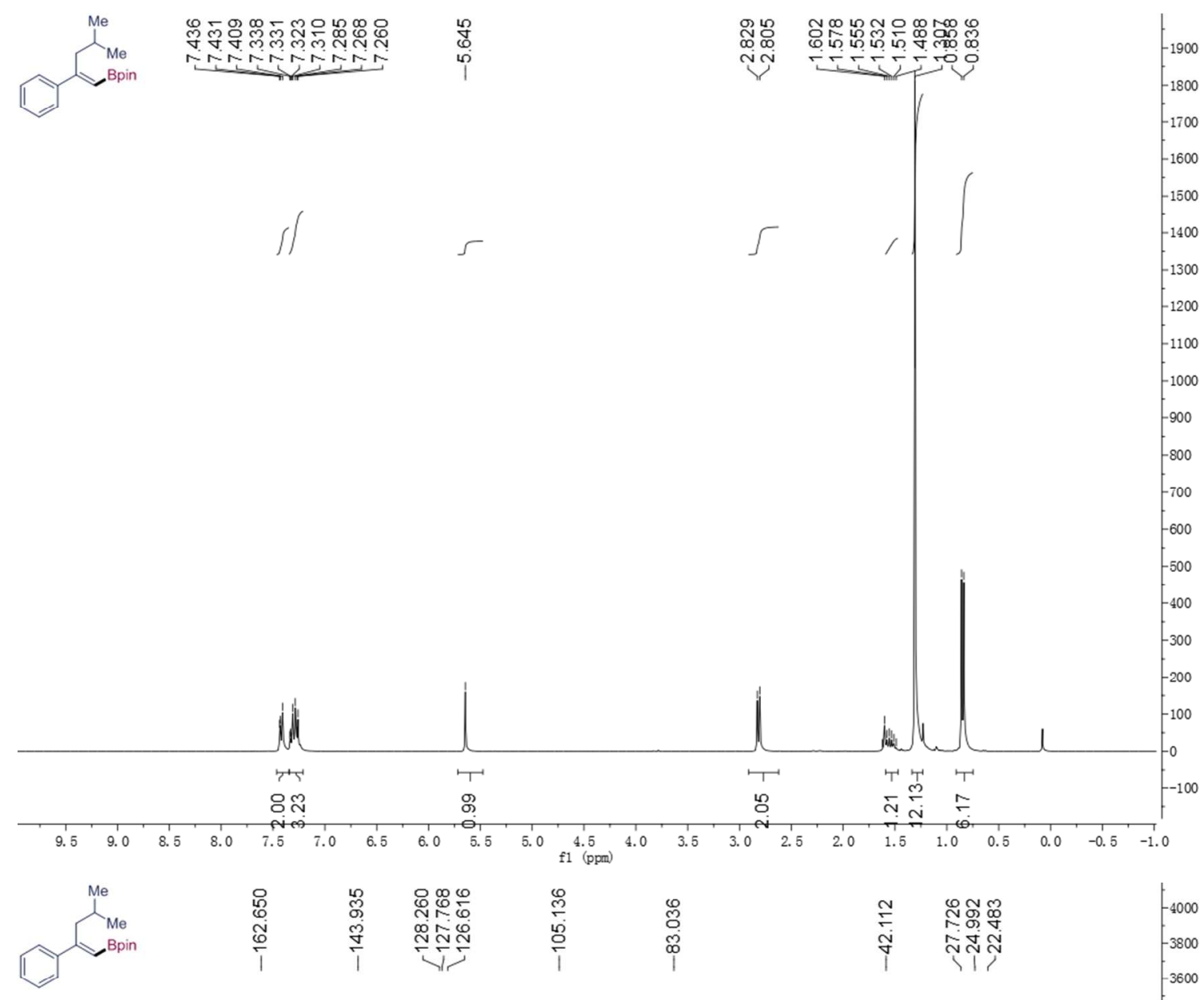

4000
3800
3600

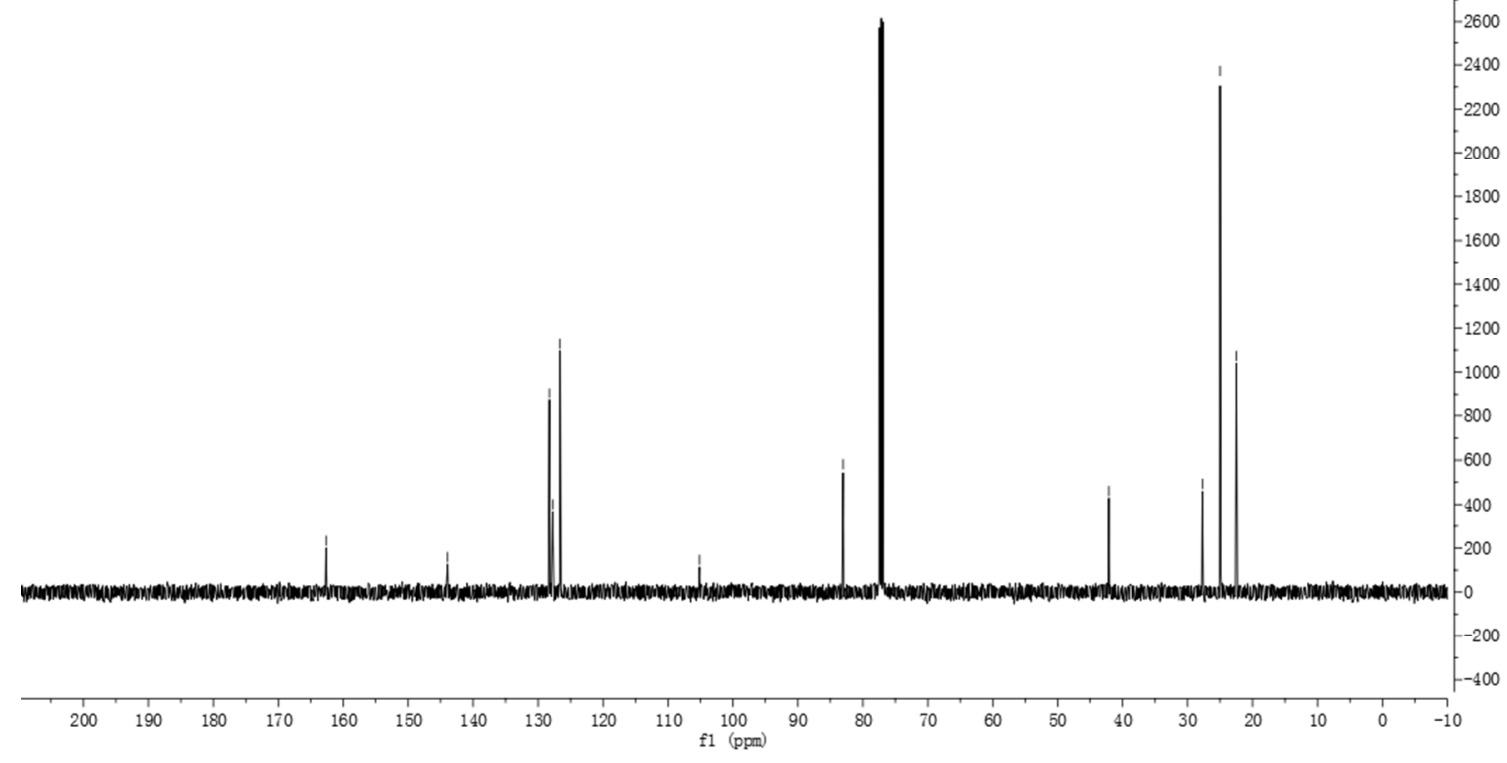




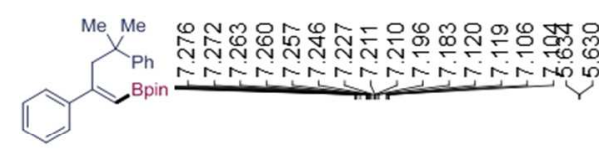

अ.
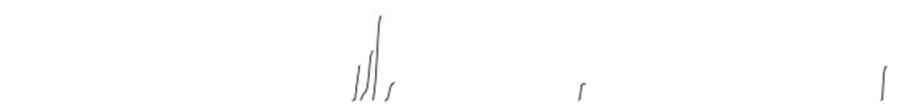

Nh

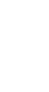

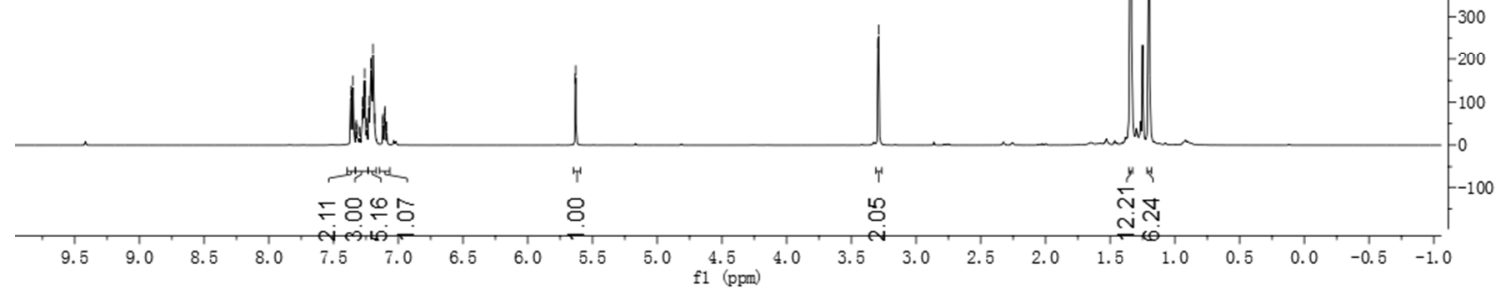

11)

$\underset{\substack{0 \\ \infty \\ \infty}}{\infty}$
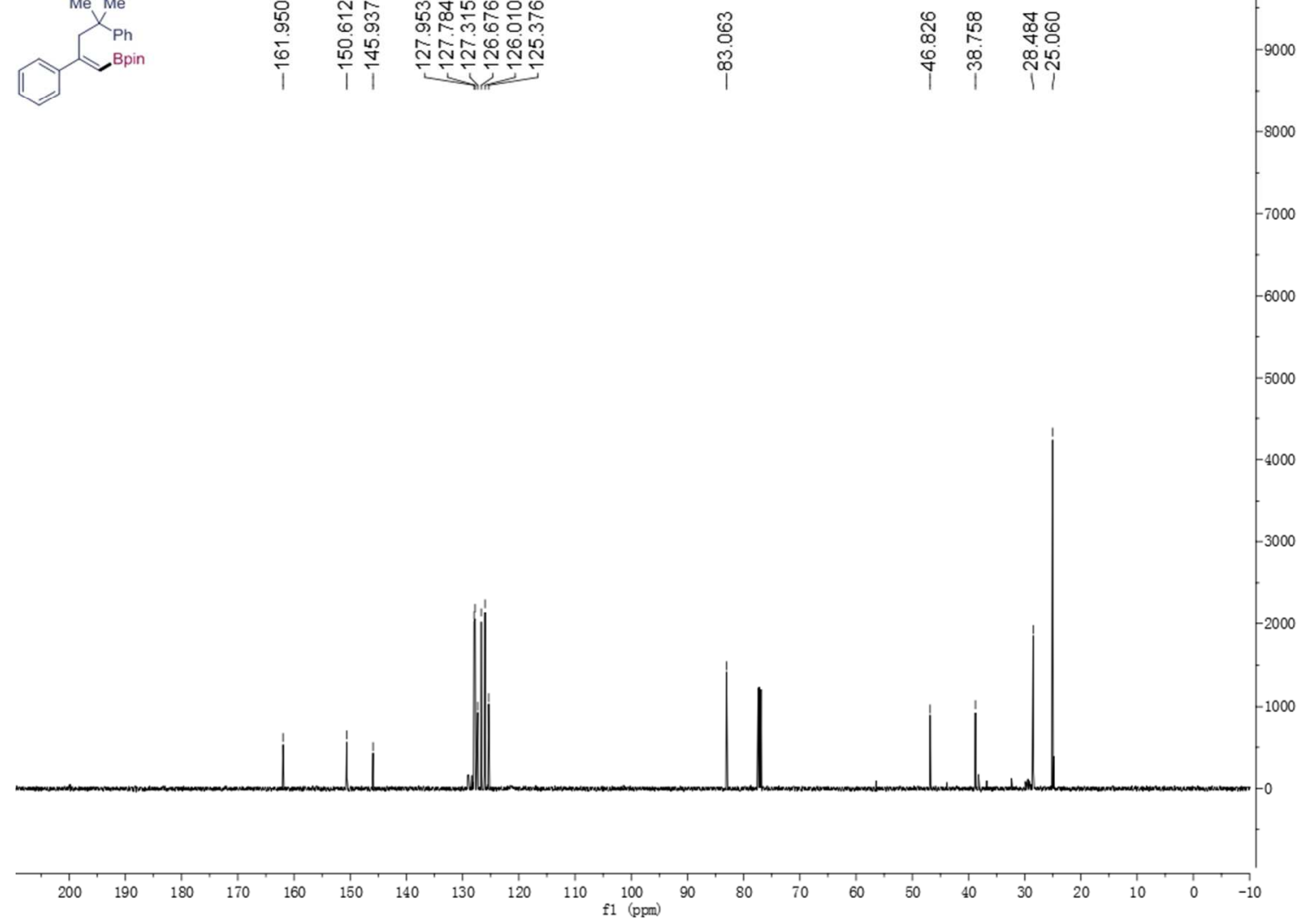


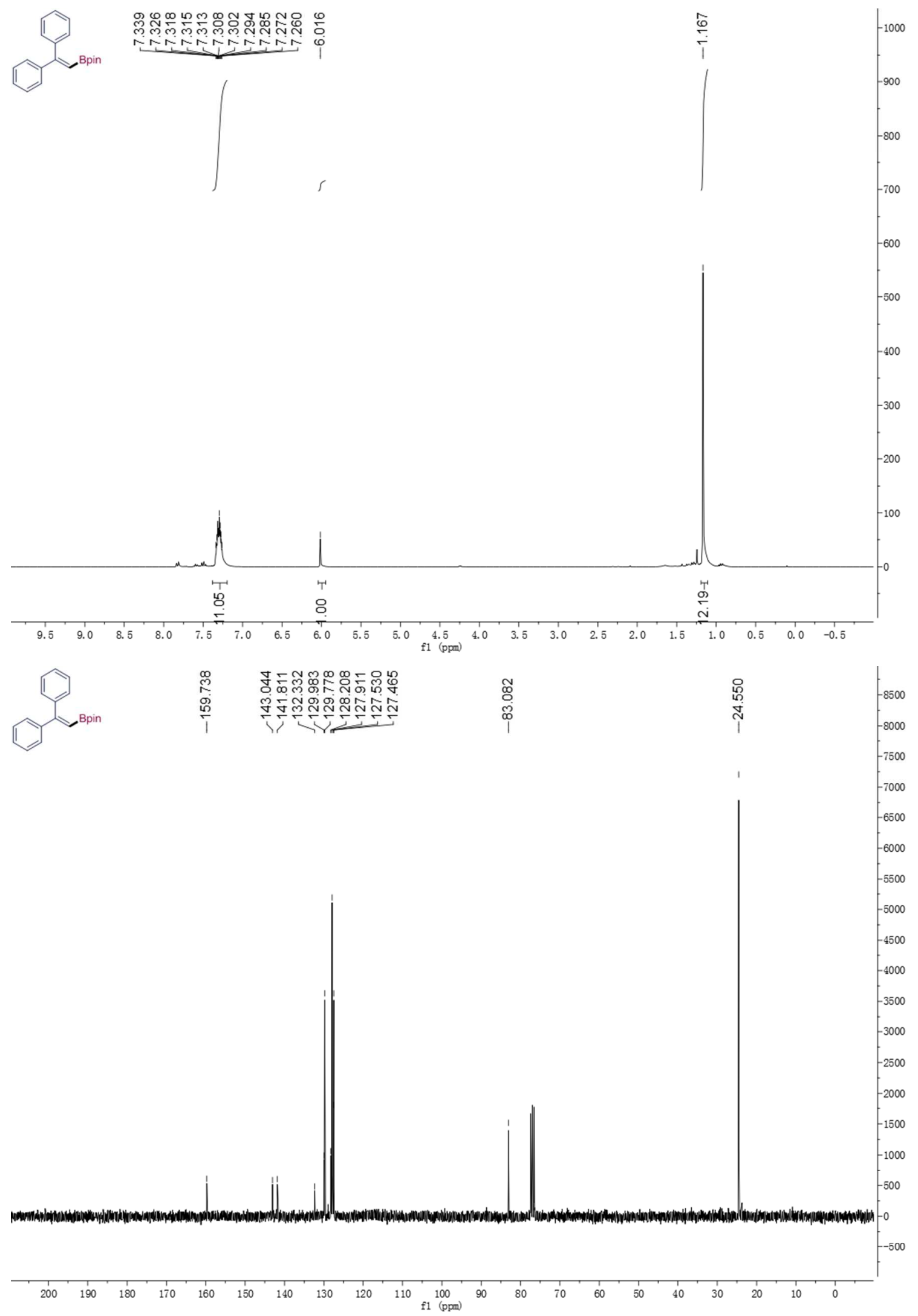




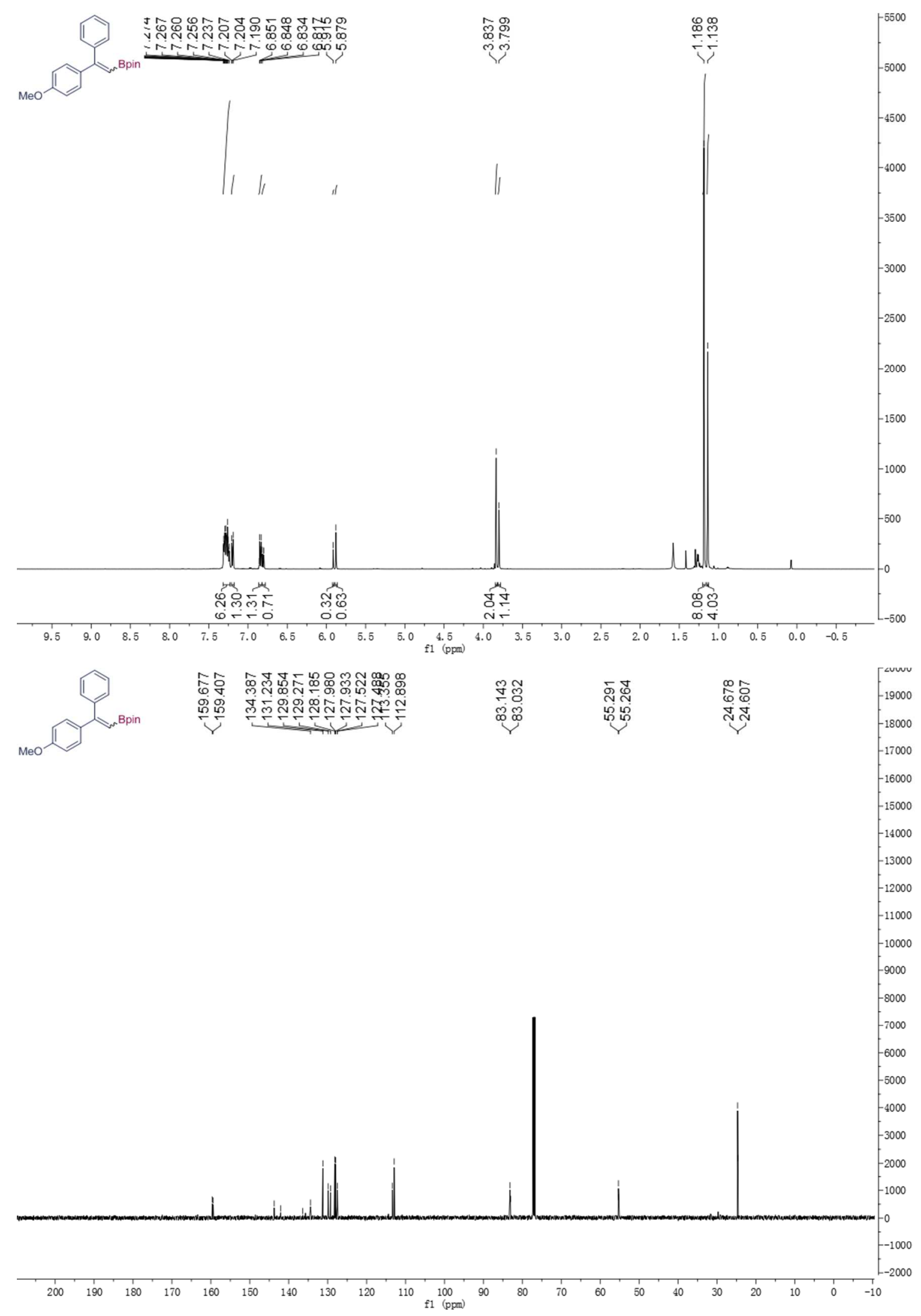




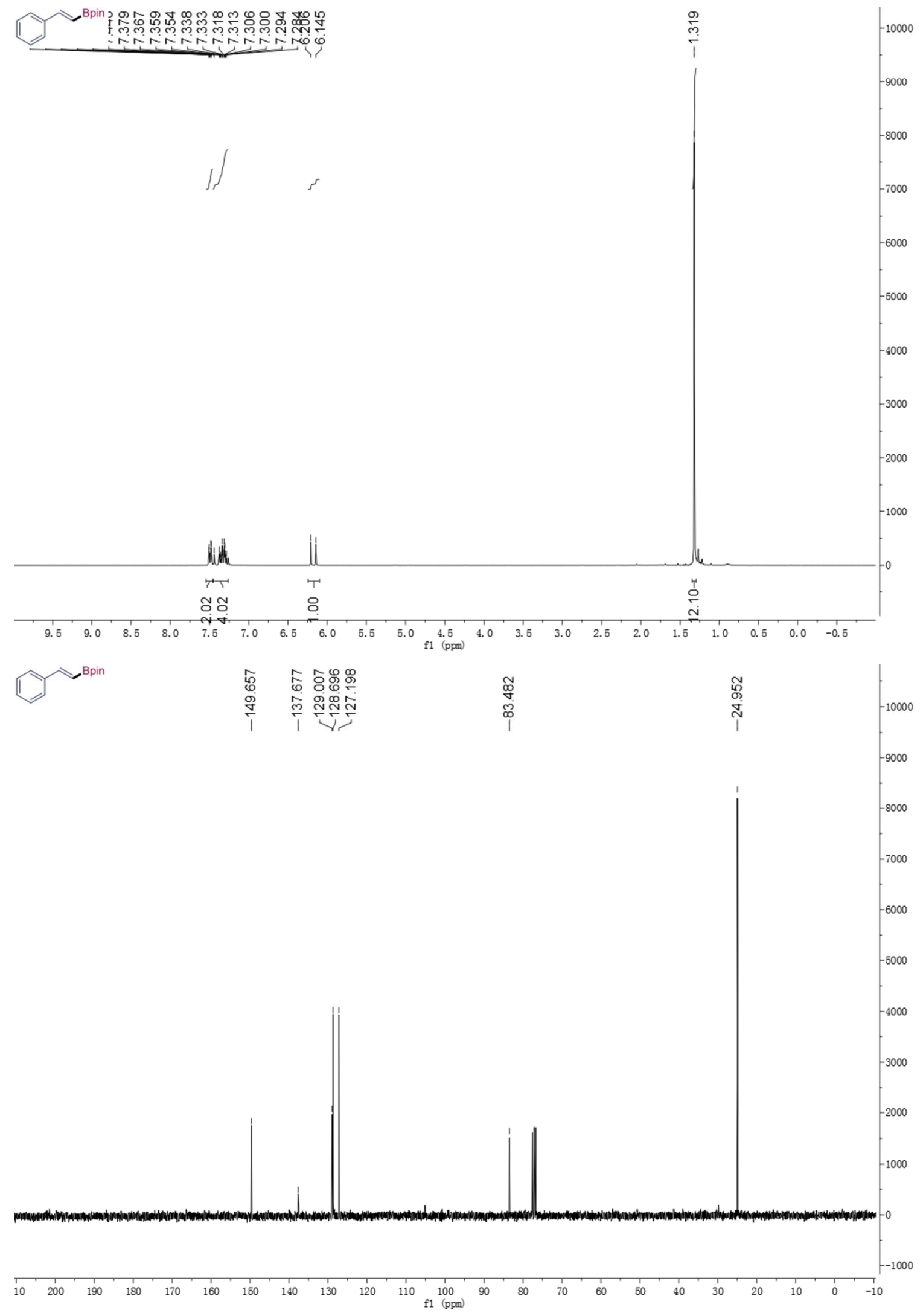


Wang, Wu, and GE, Supporting Information

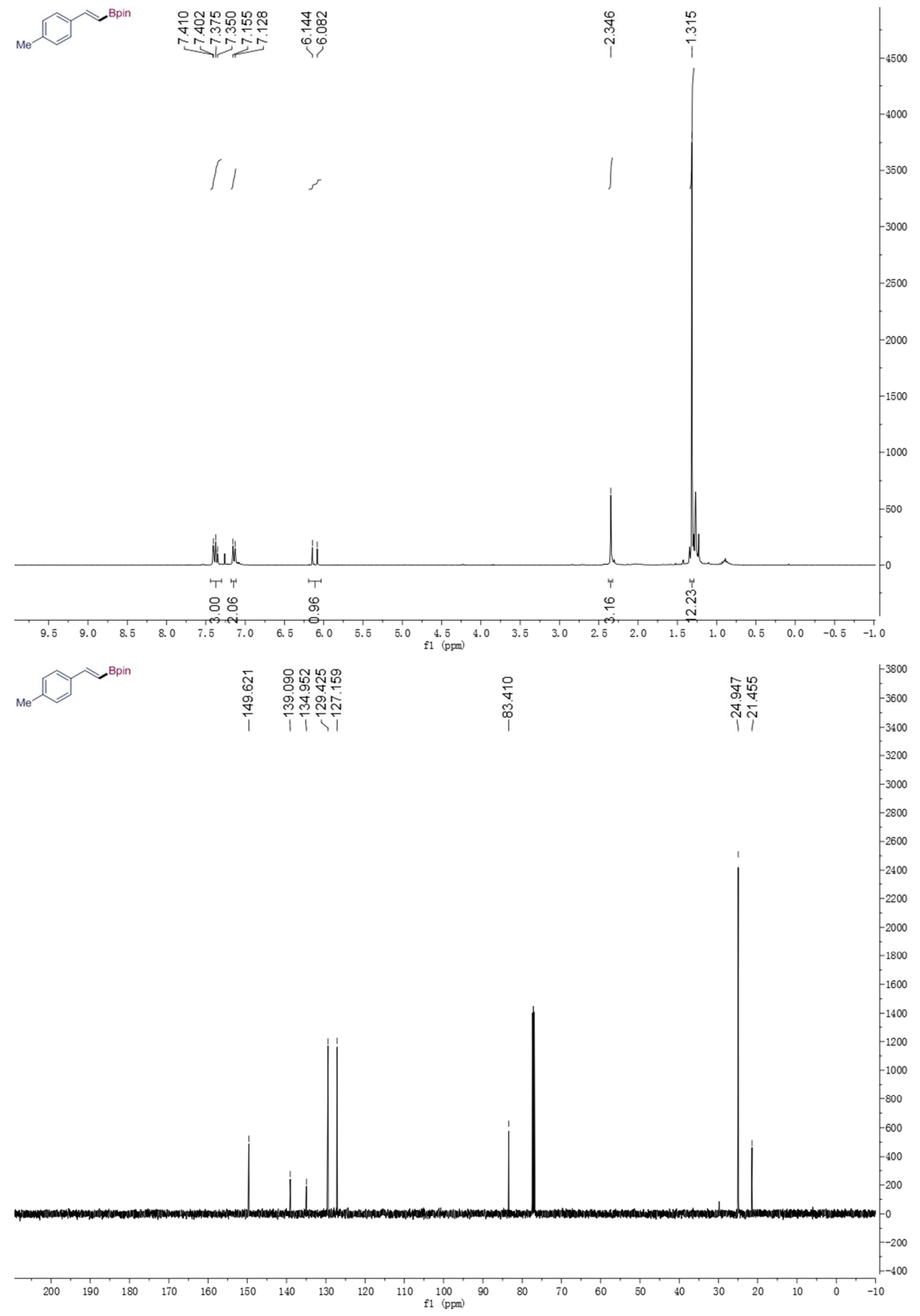


Wang, Wu, and GE, Supporting Information

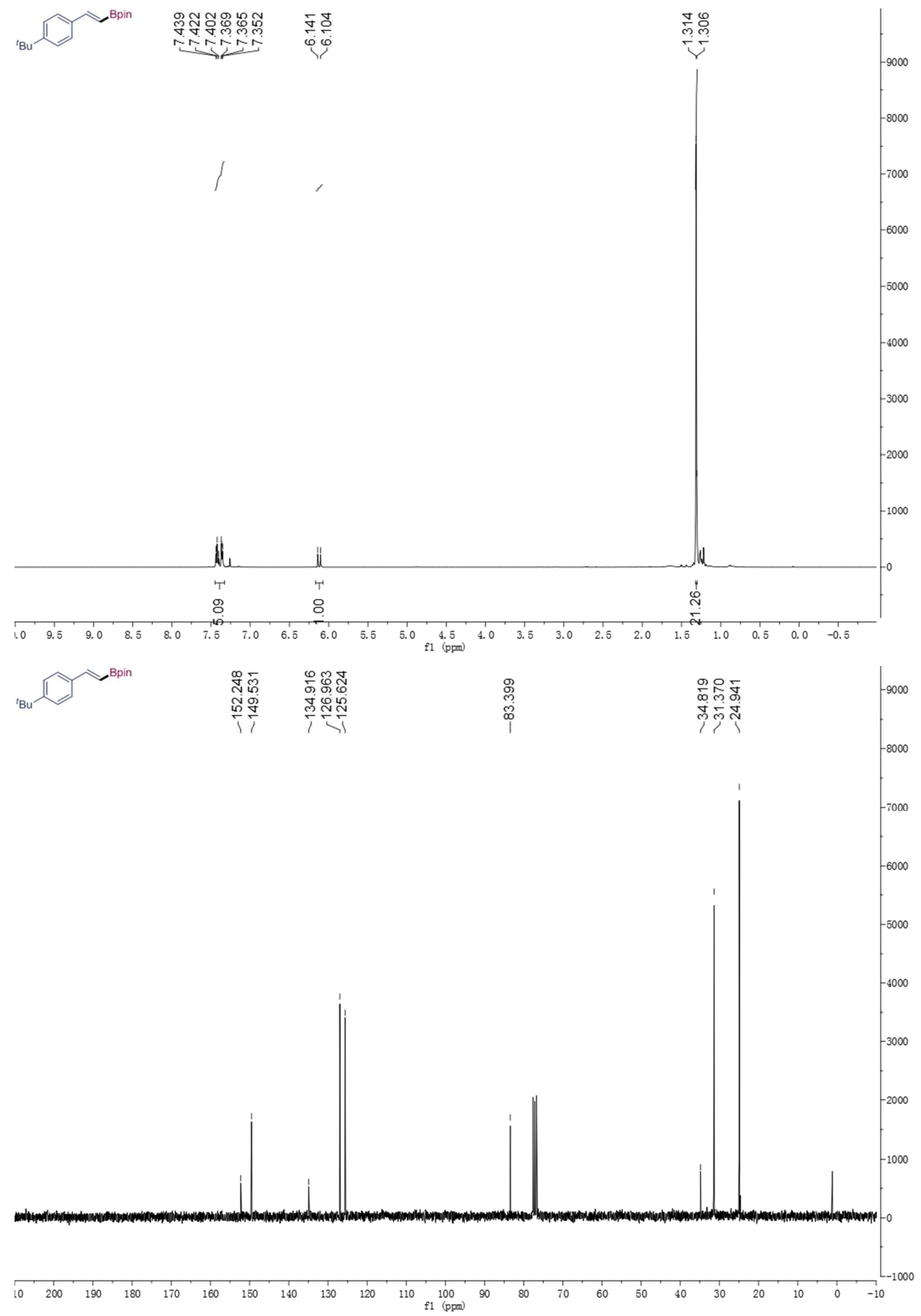




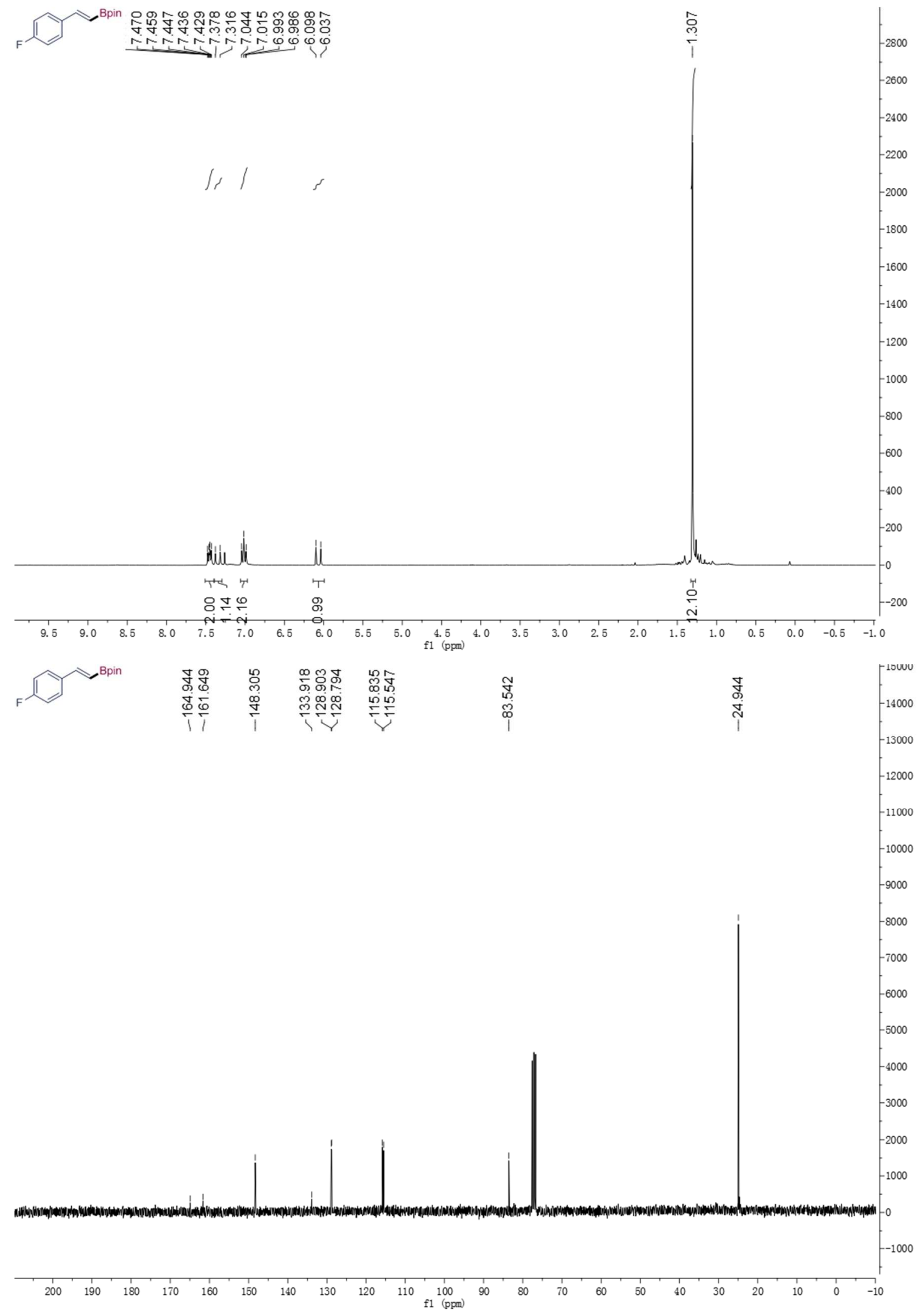


Wang, Wu, and GE, Supporting Information

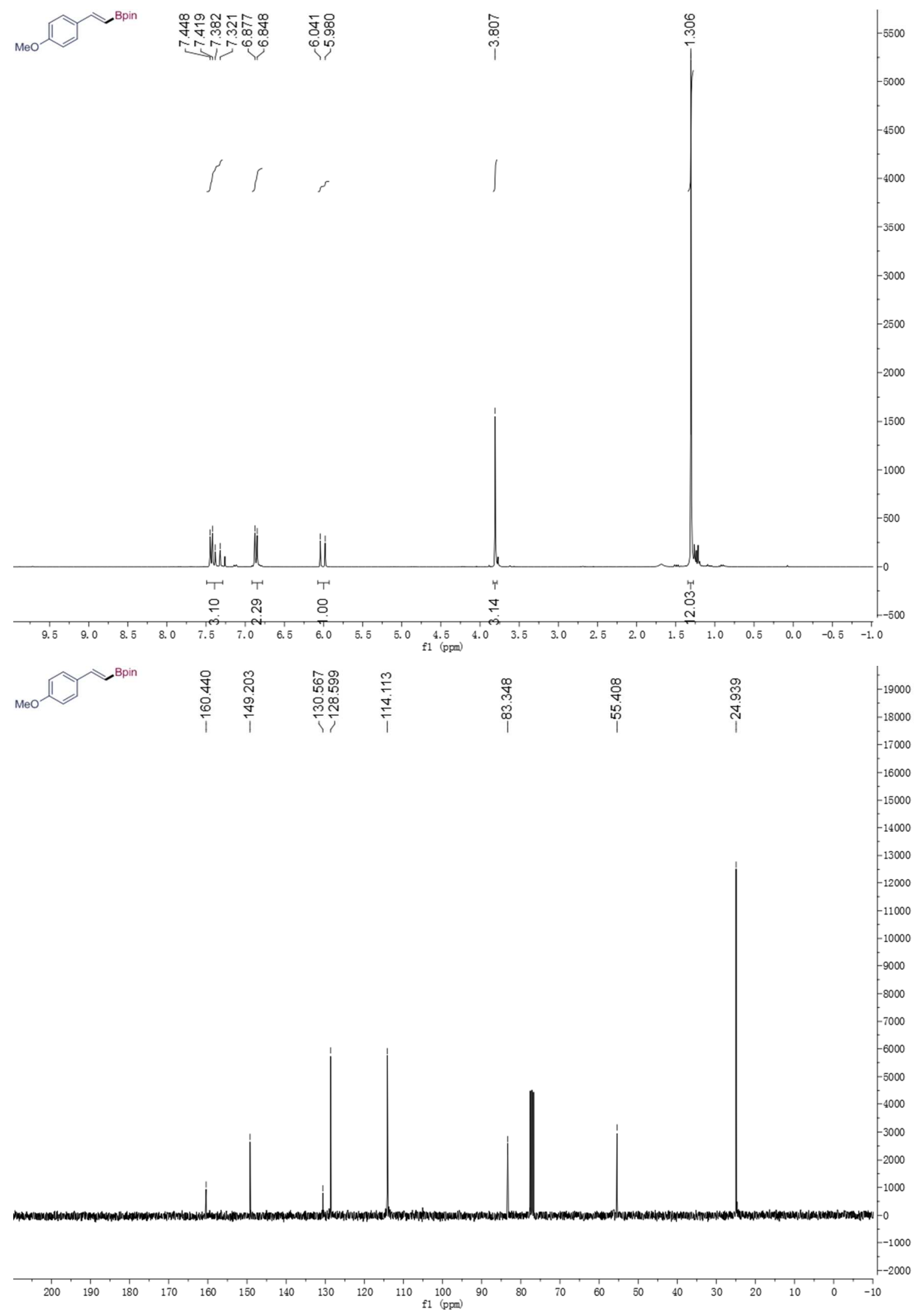


Wang, Wu, and GE, Supporting Information

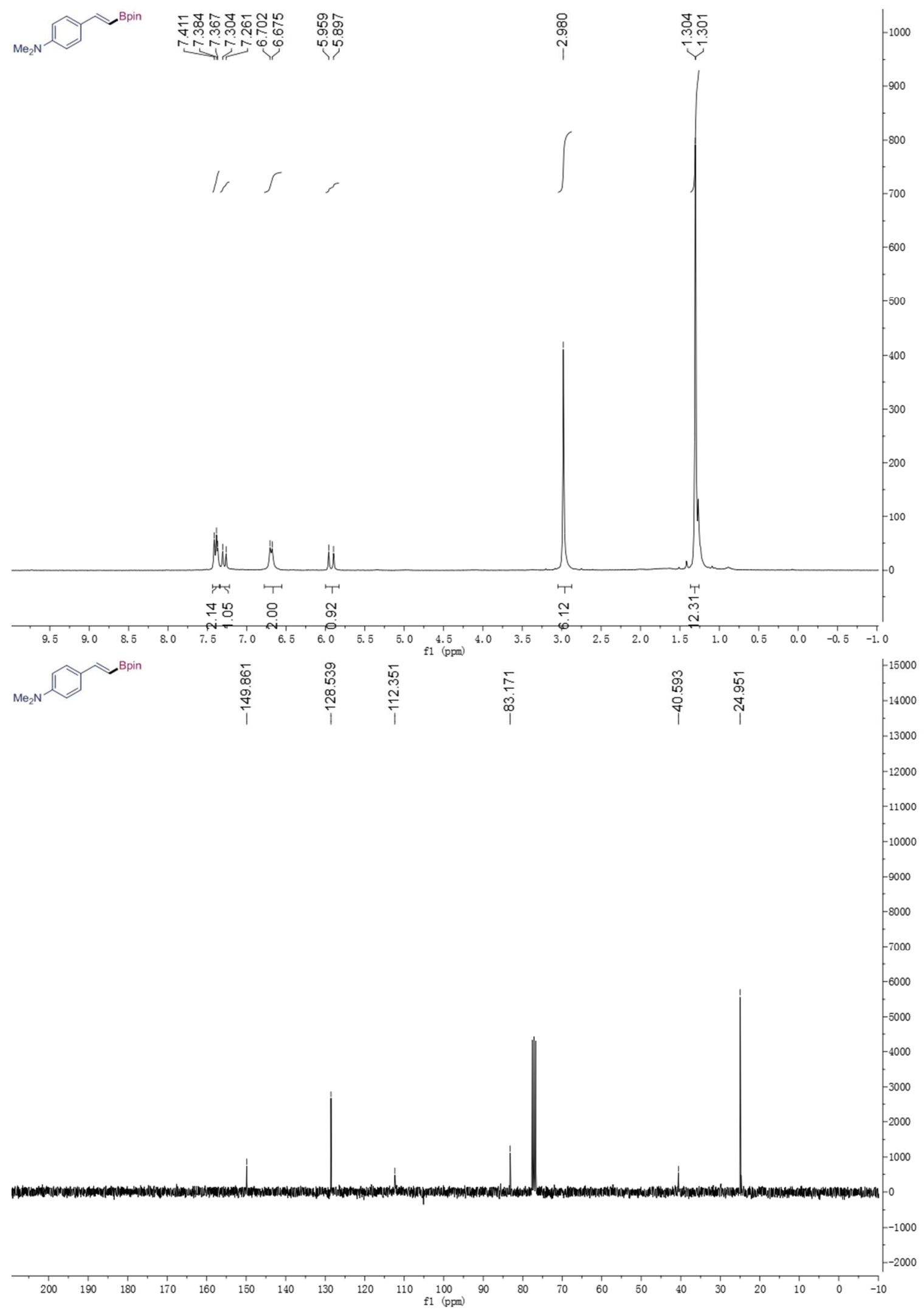




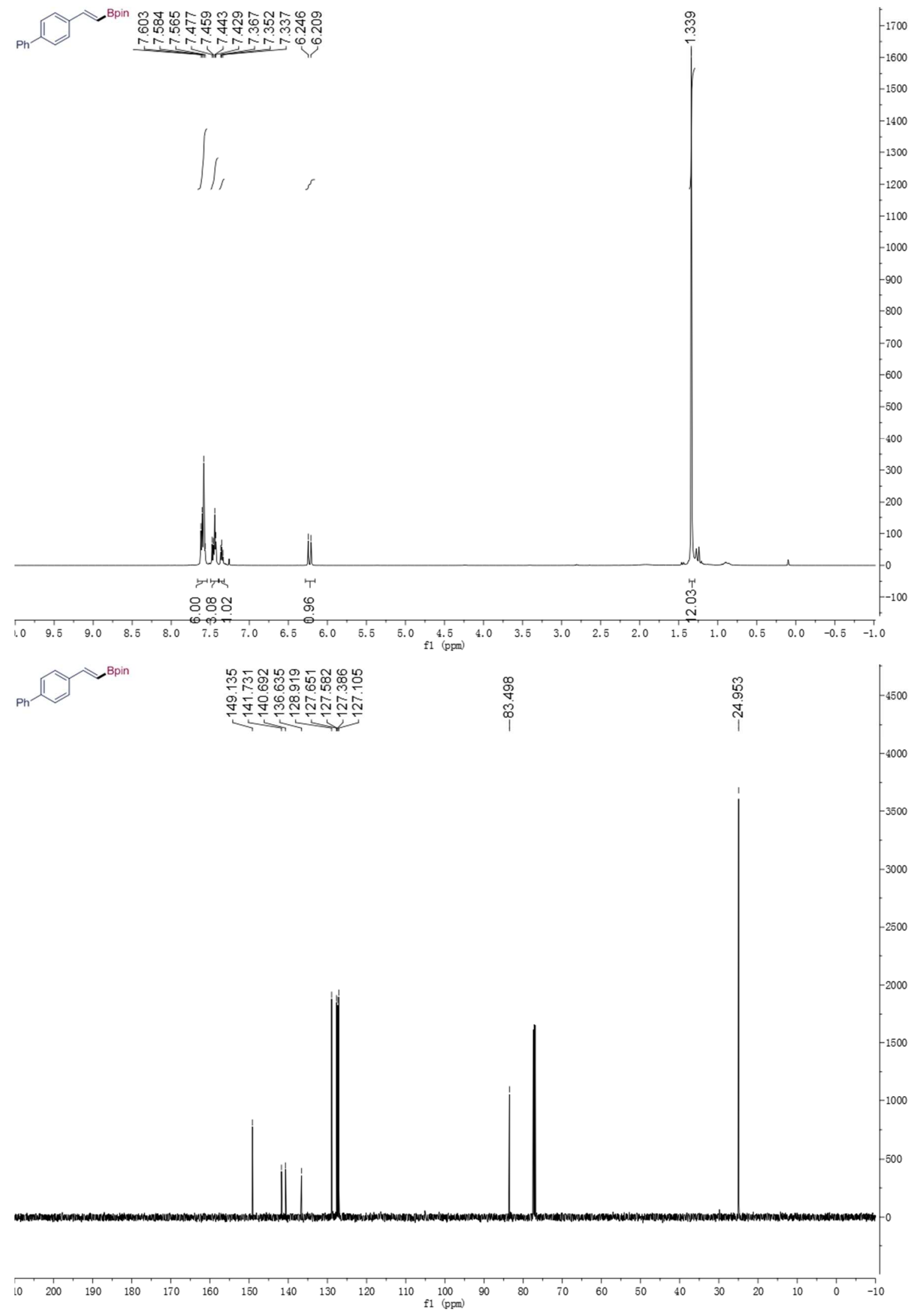


Wang, Wu, and GE, Supporting Information

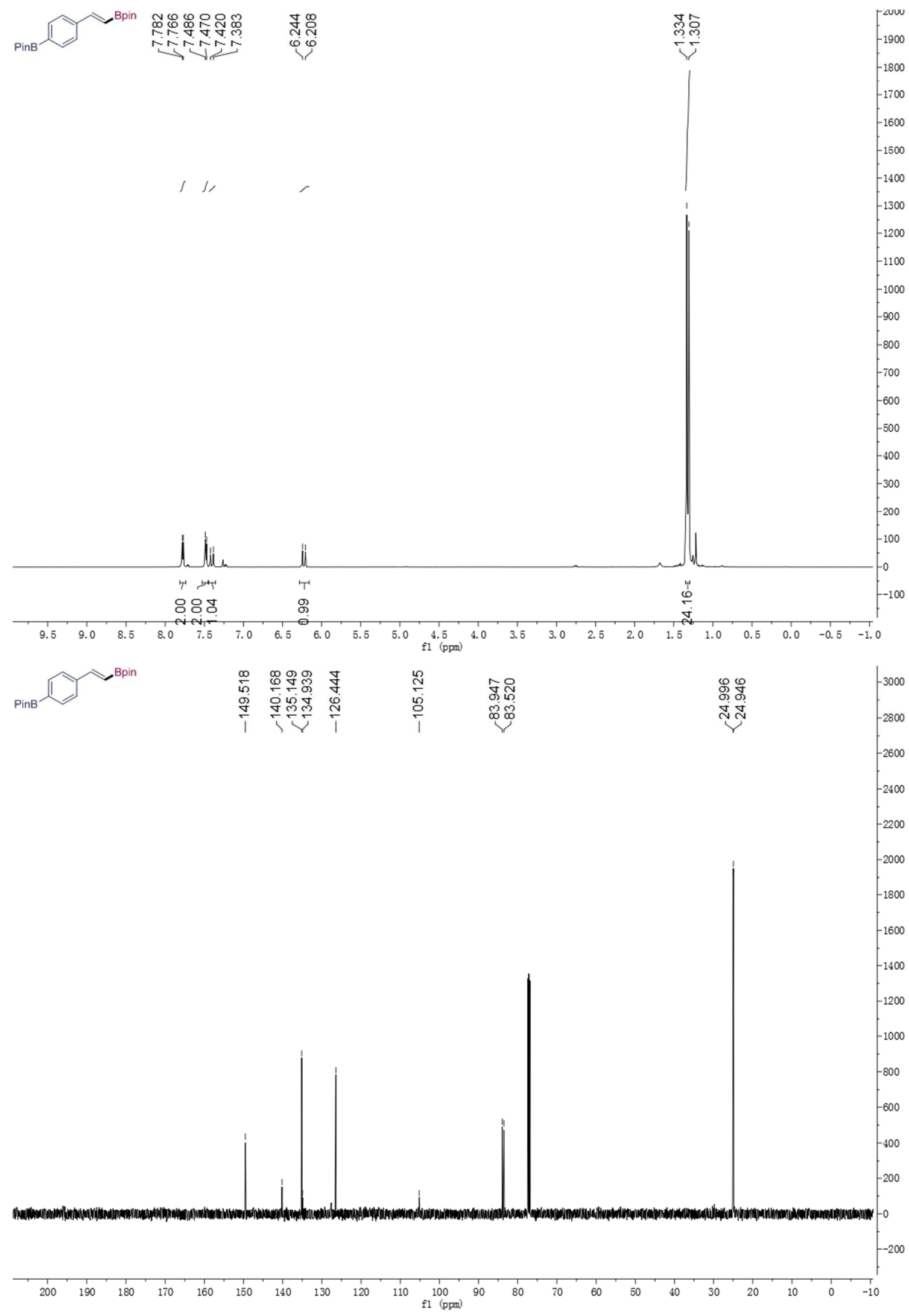




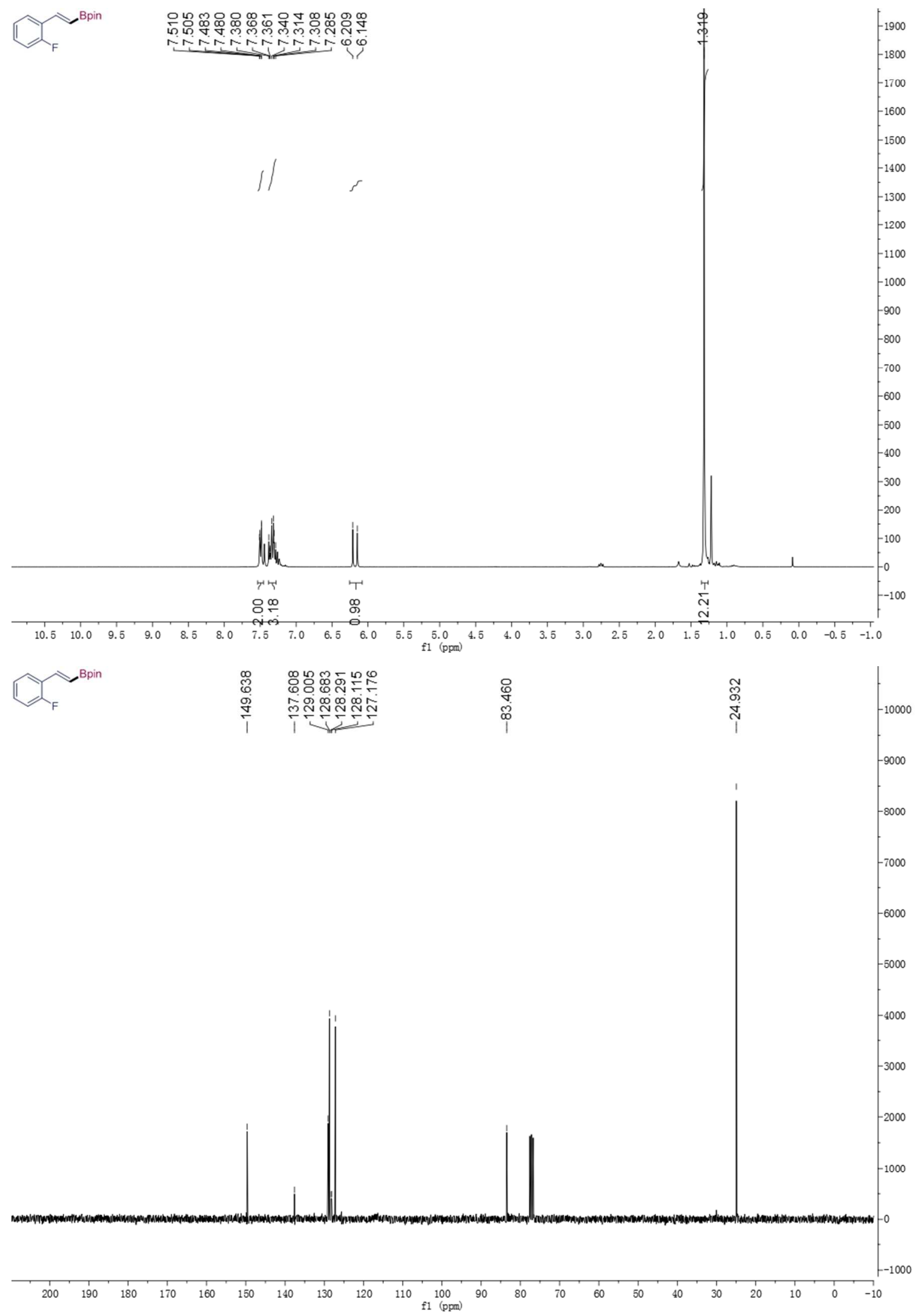


Wang, Wu, and GE, Supporting Information

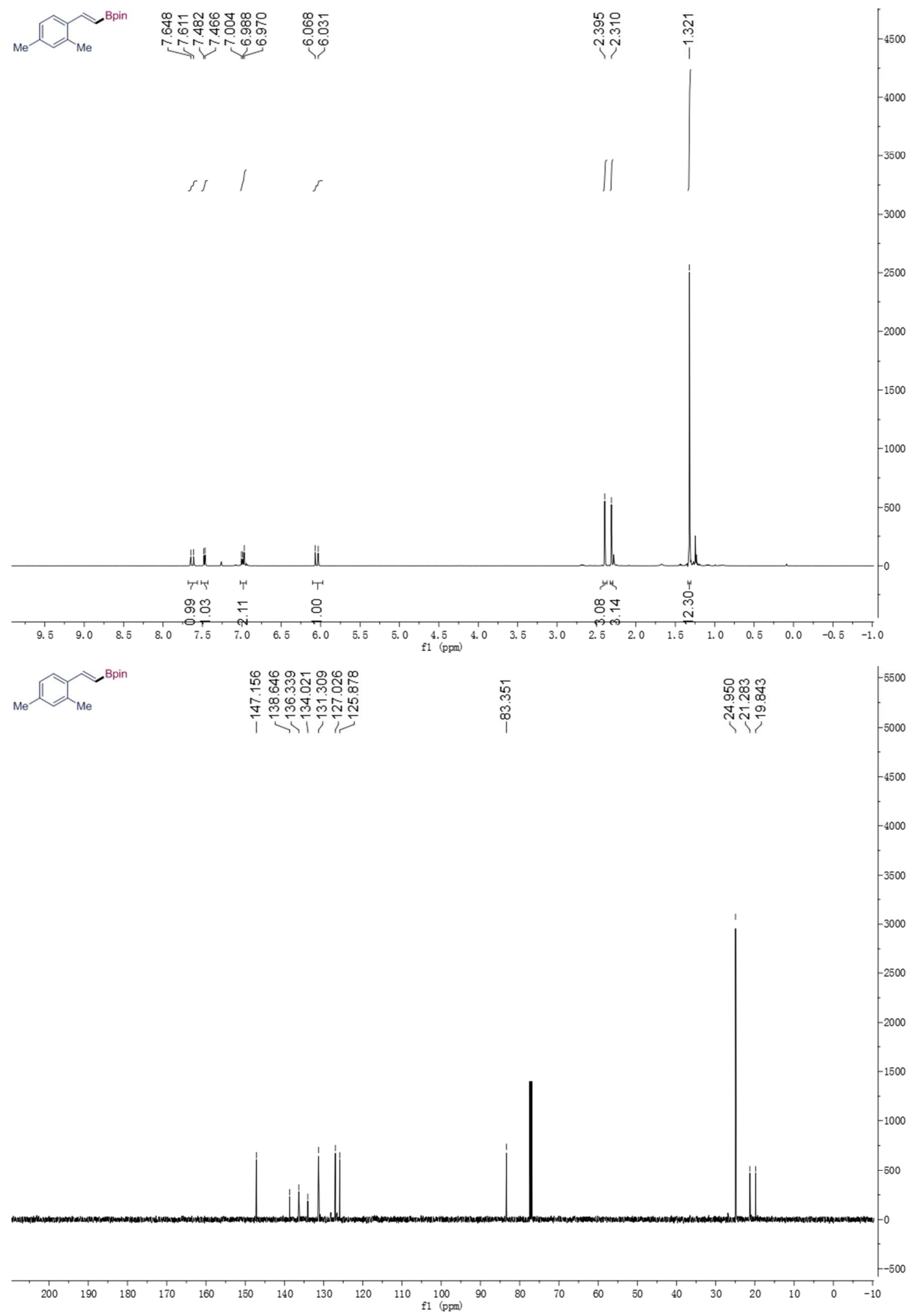




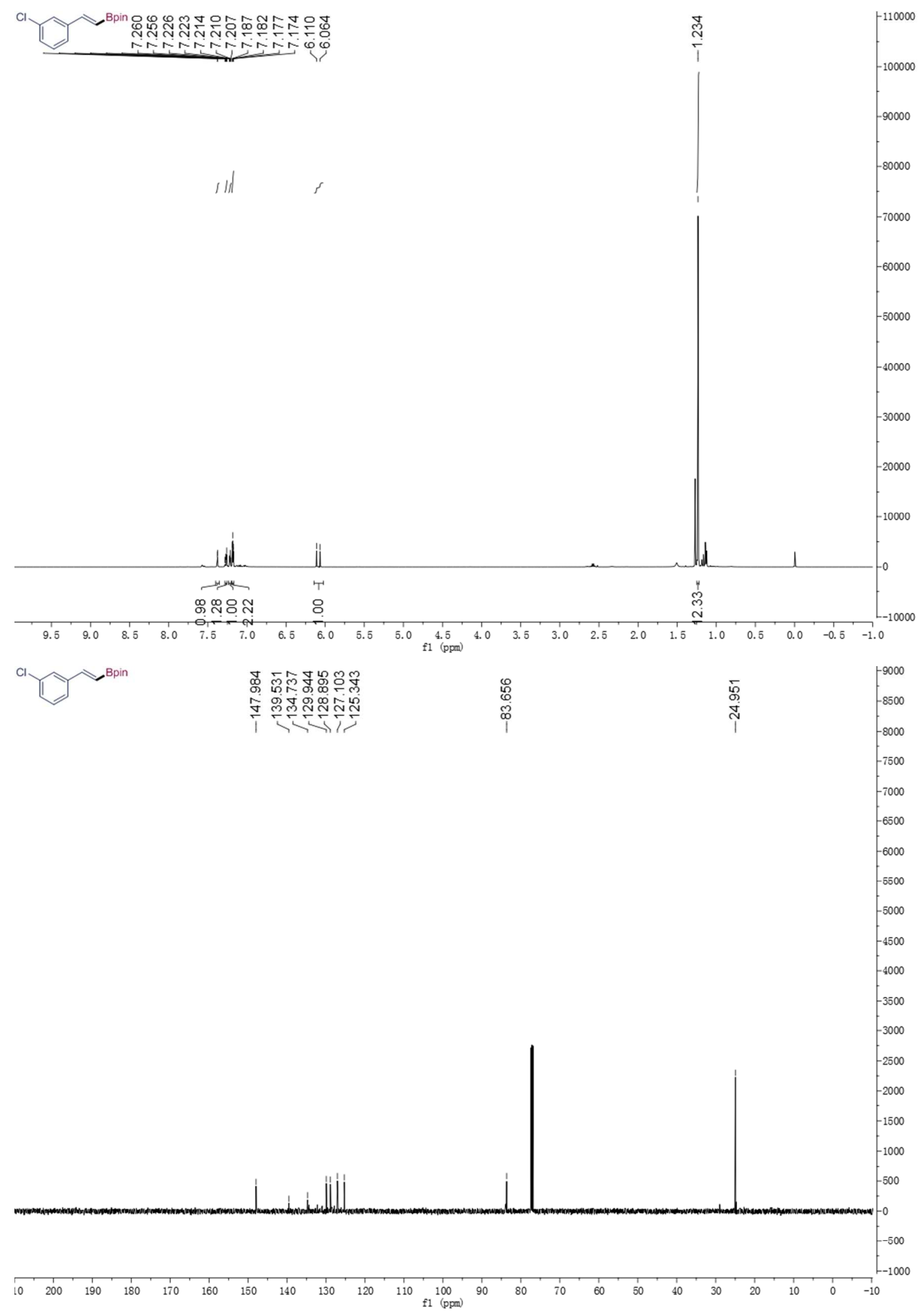


Wang, Wu, and GE, Supporting Information
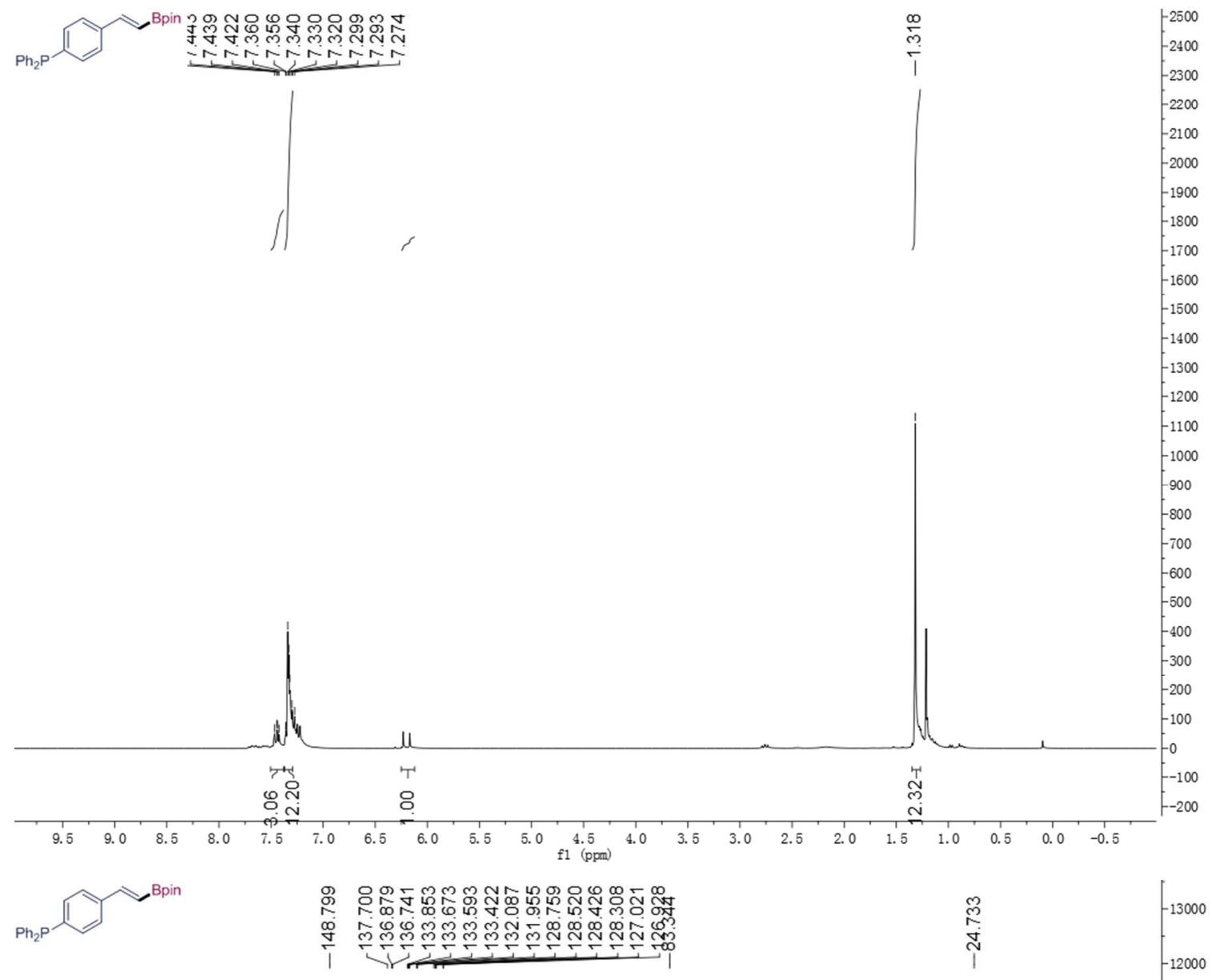

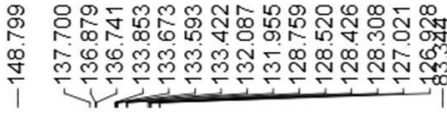
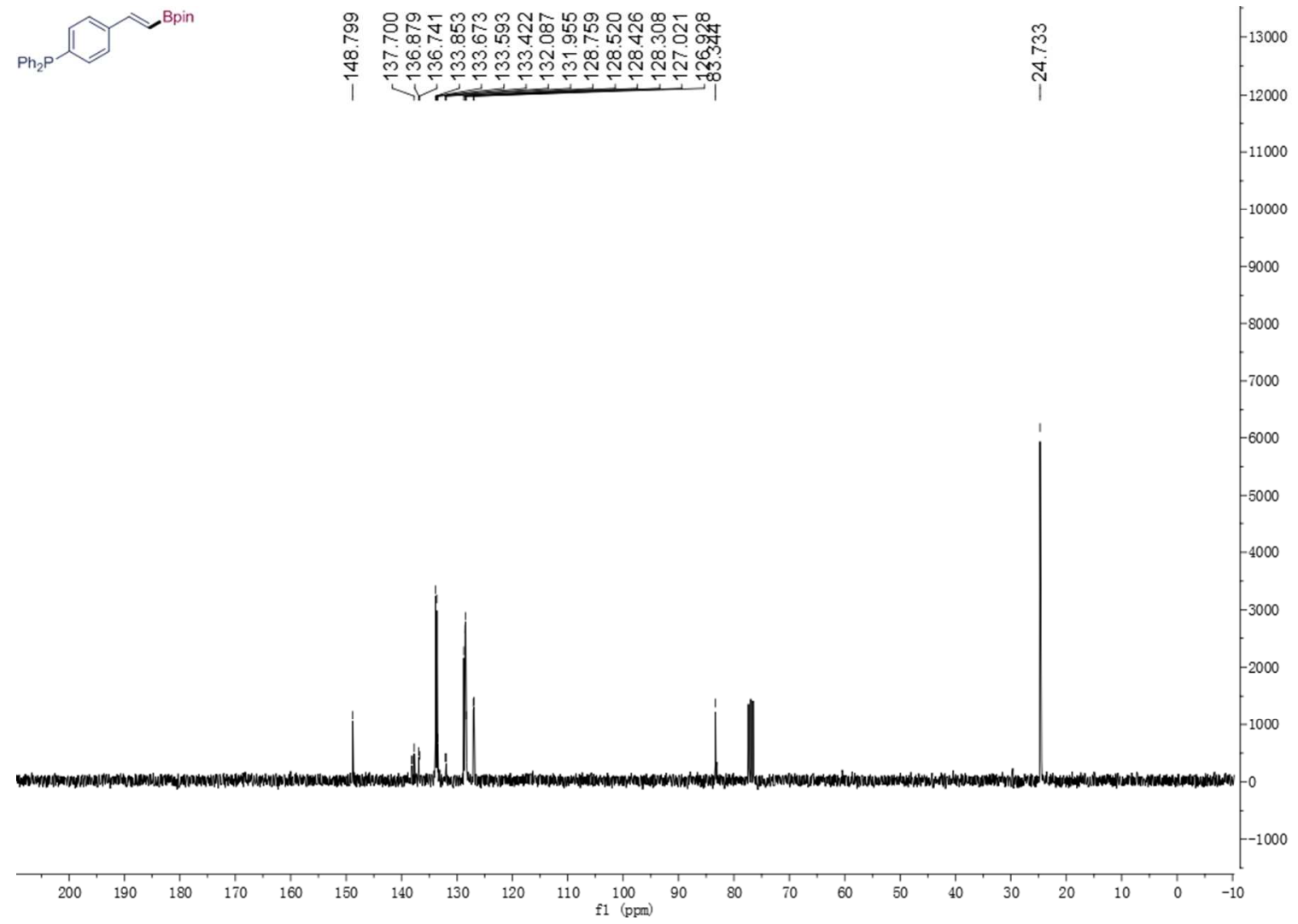
Wang, Wu, and GE, Supporting Information

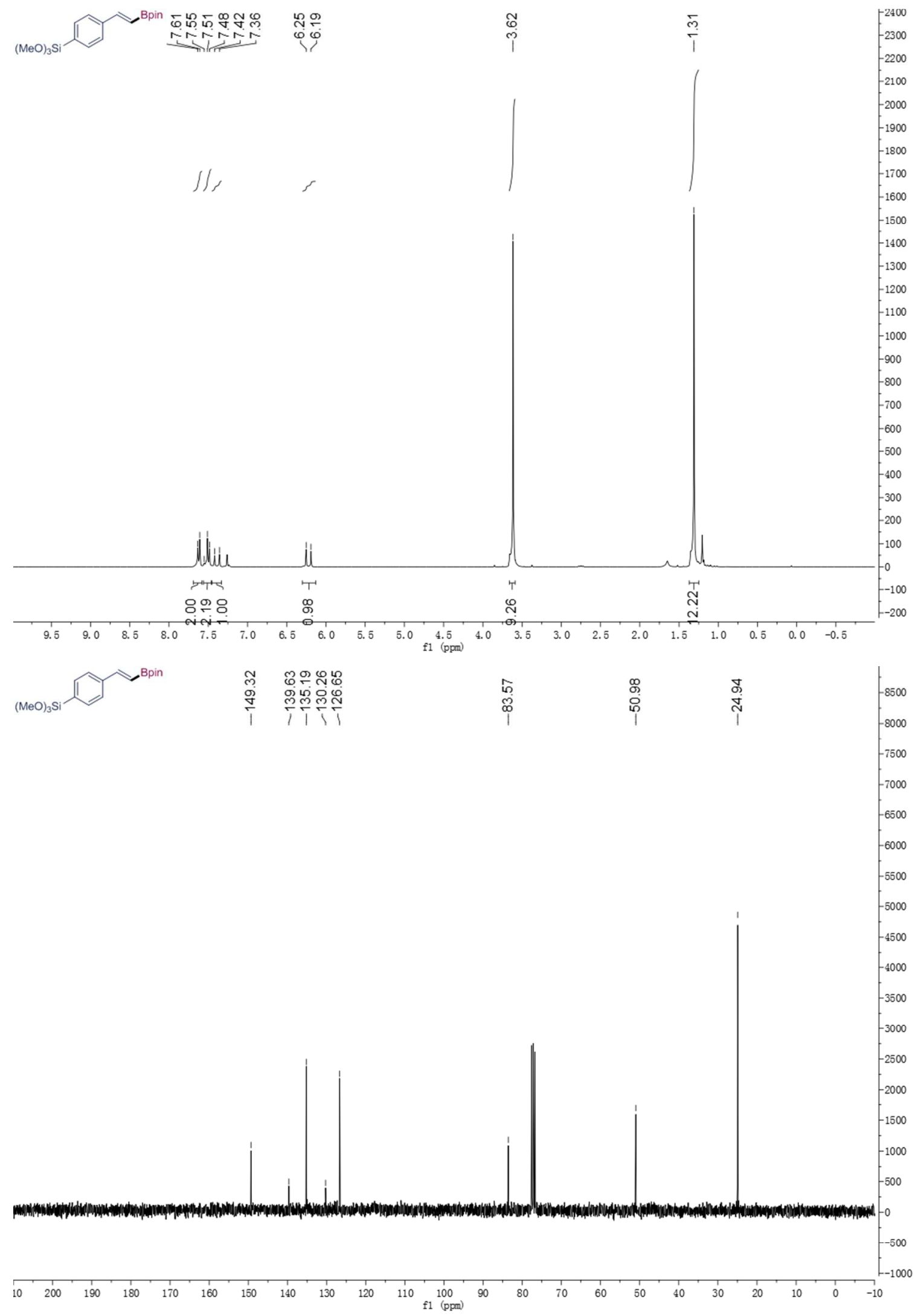




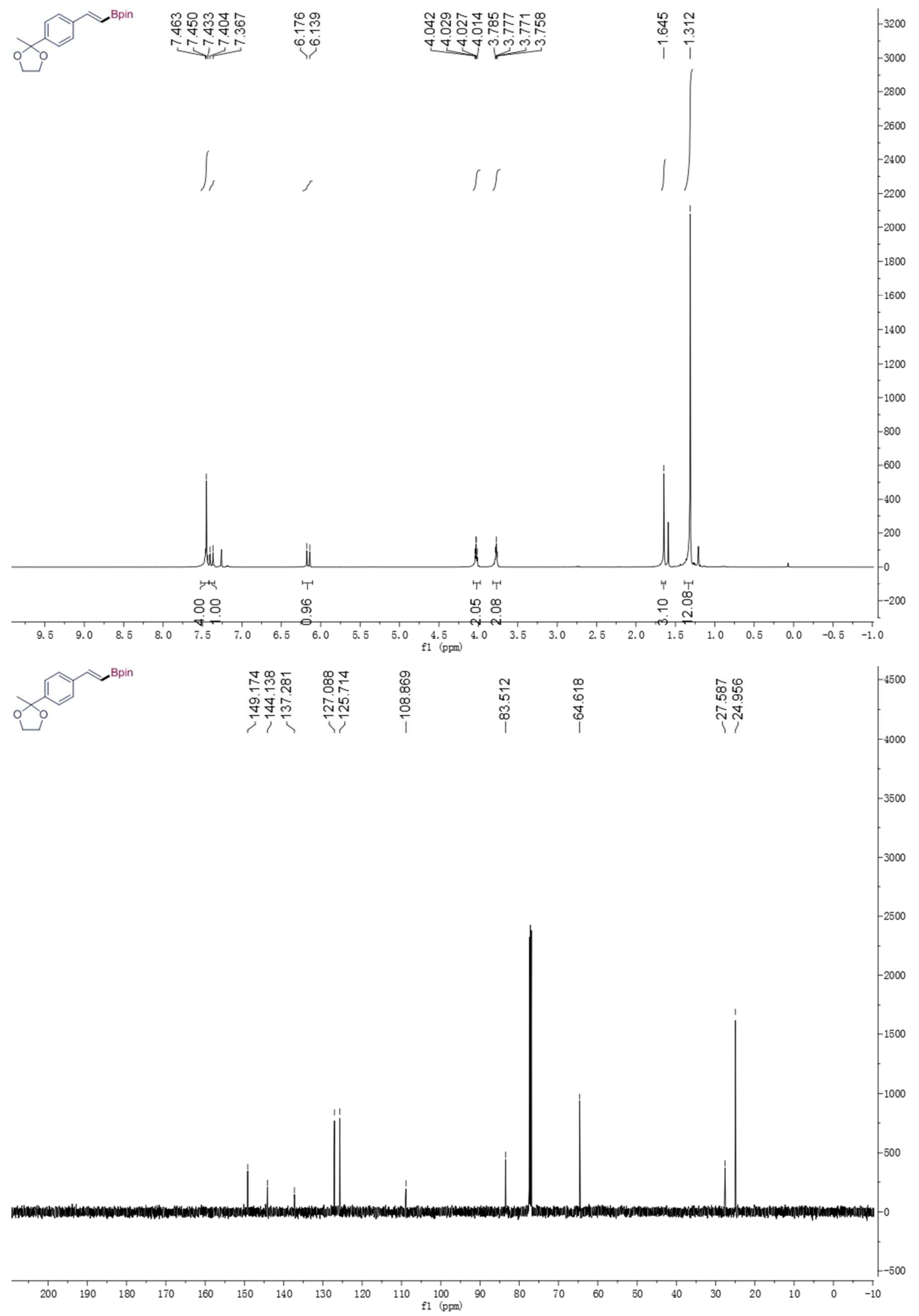


Wang, Wu, and GE, Supporting Information

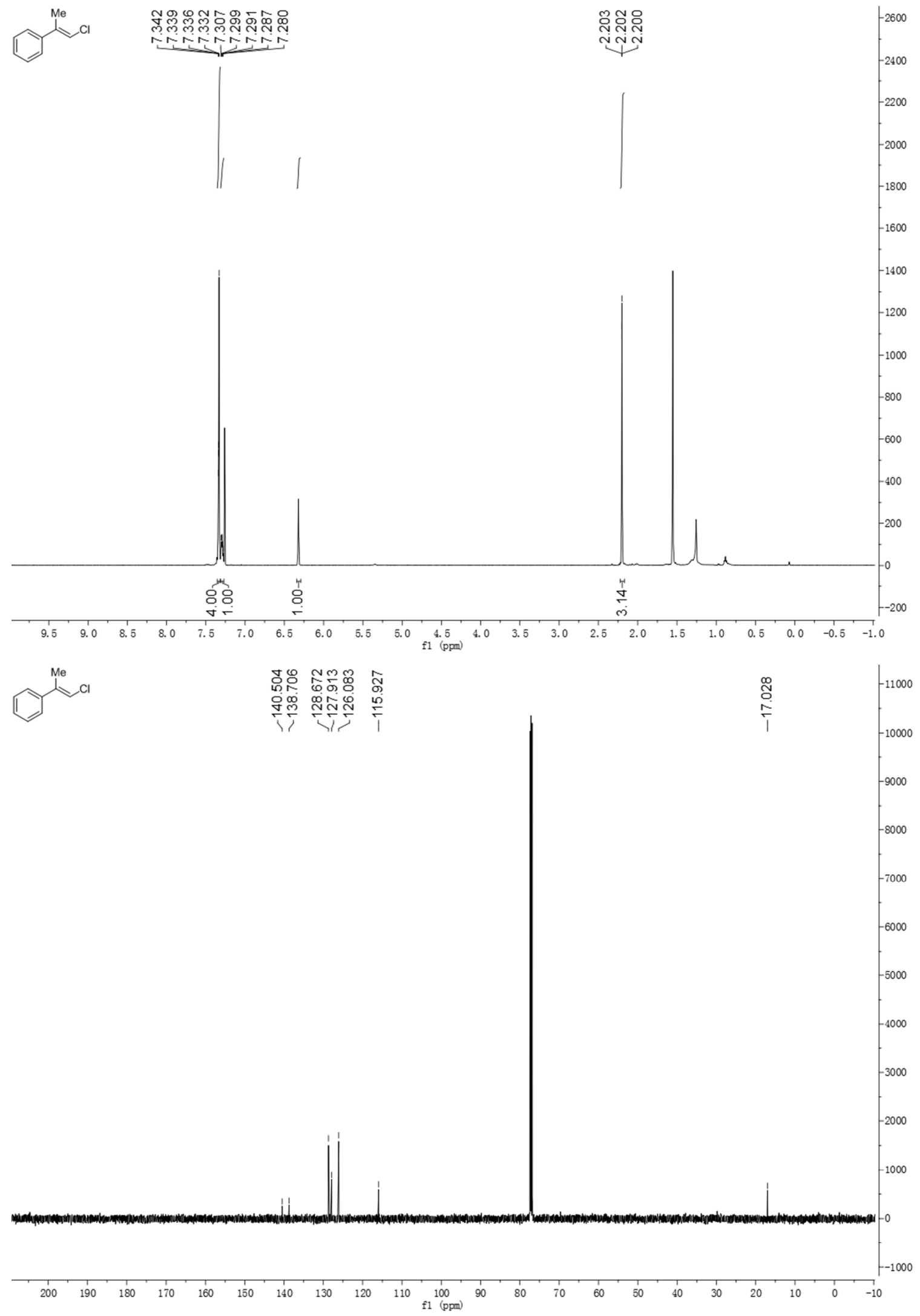


Wang, Wu, and GE, Supporting Information

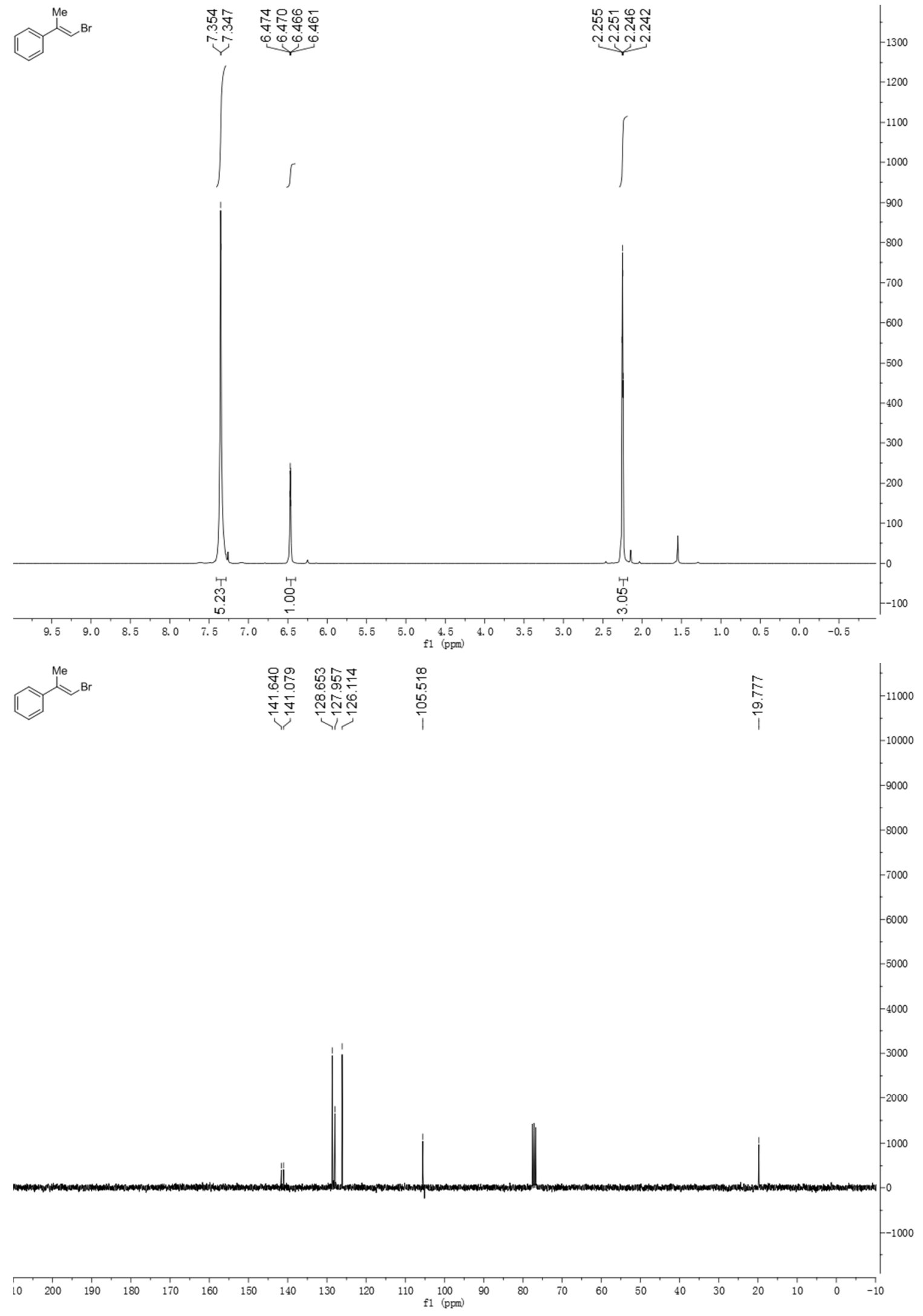




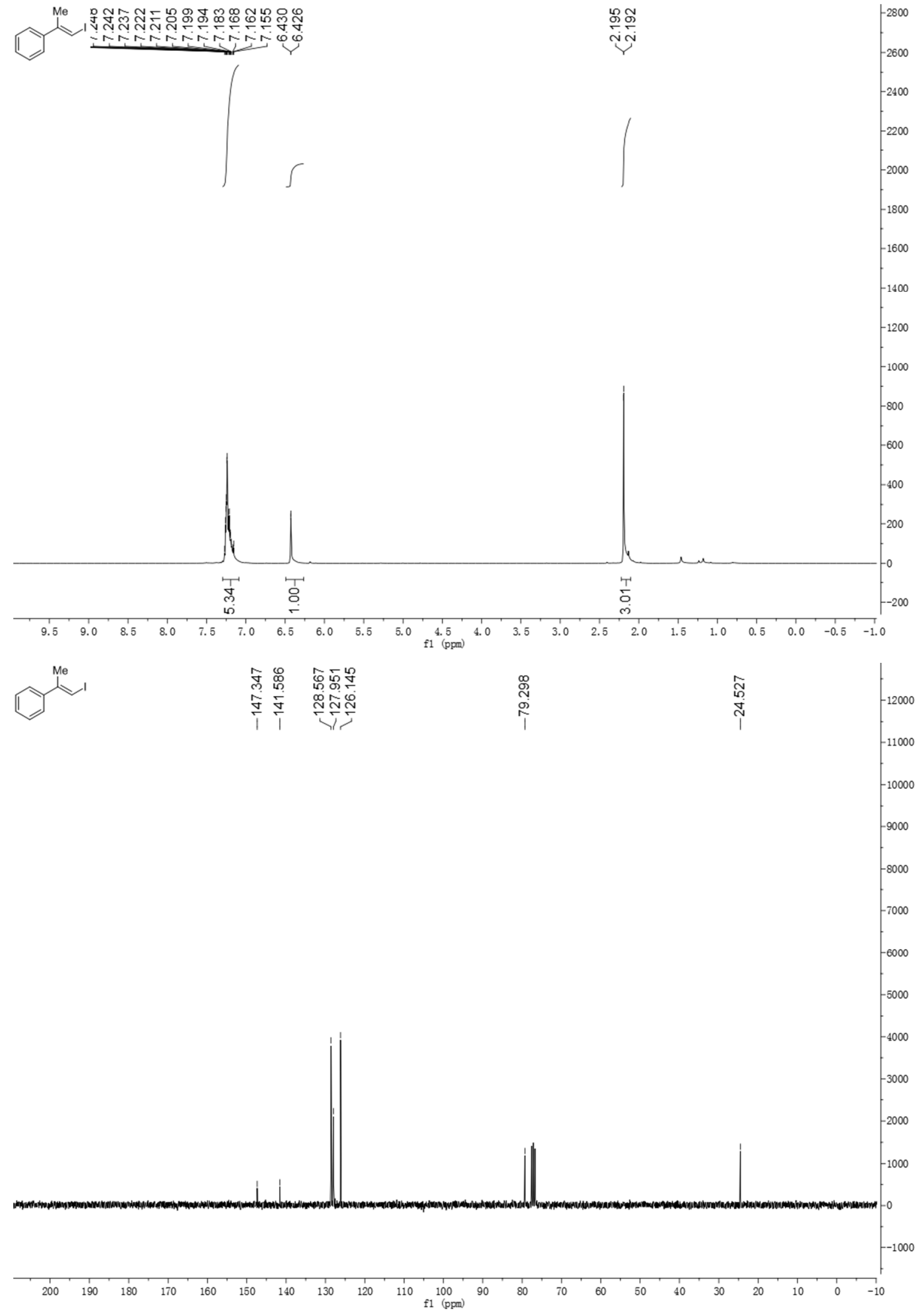




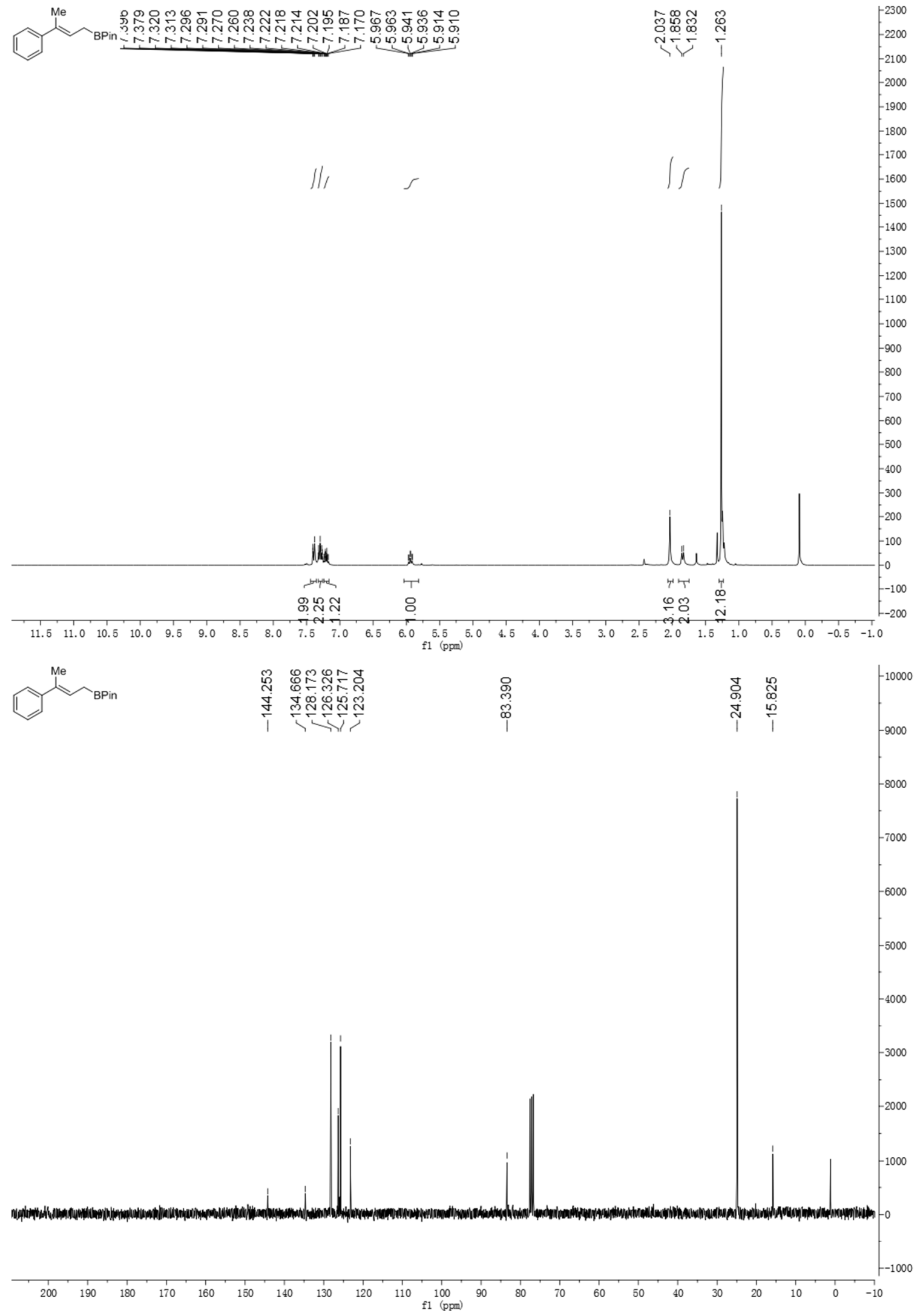


Wang, Wu, and GE, Supporting Information

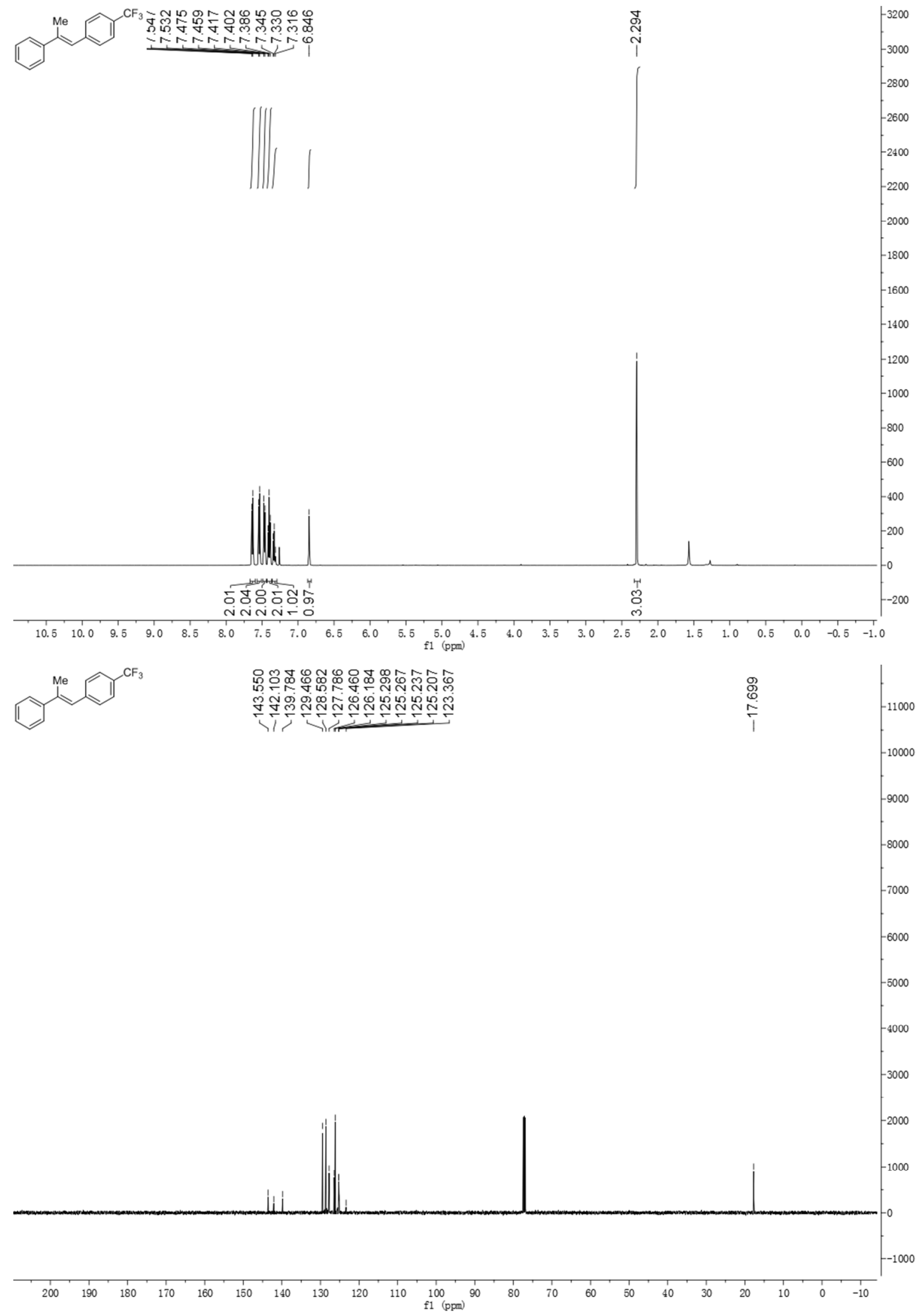




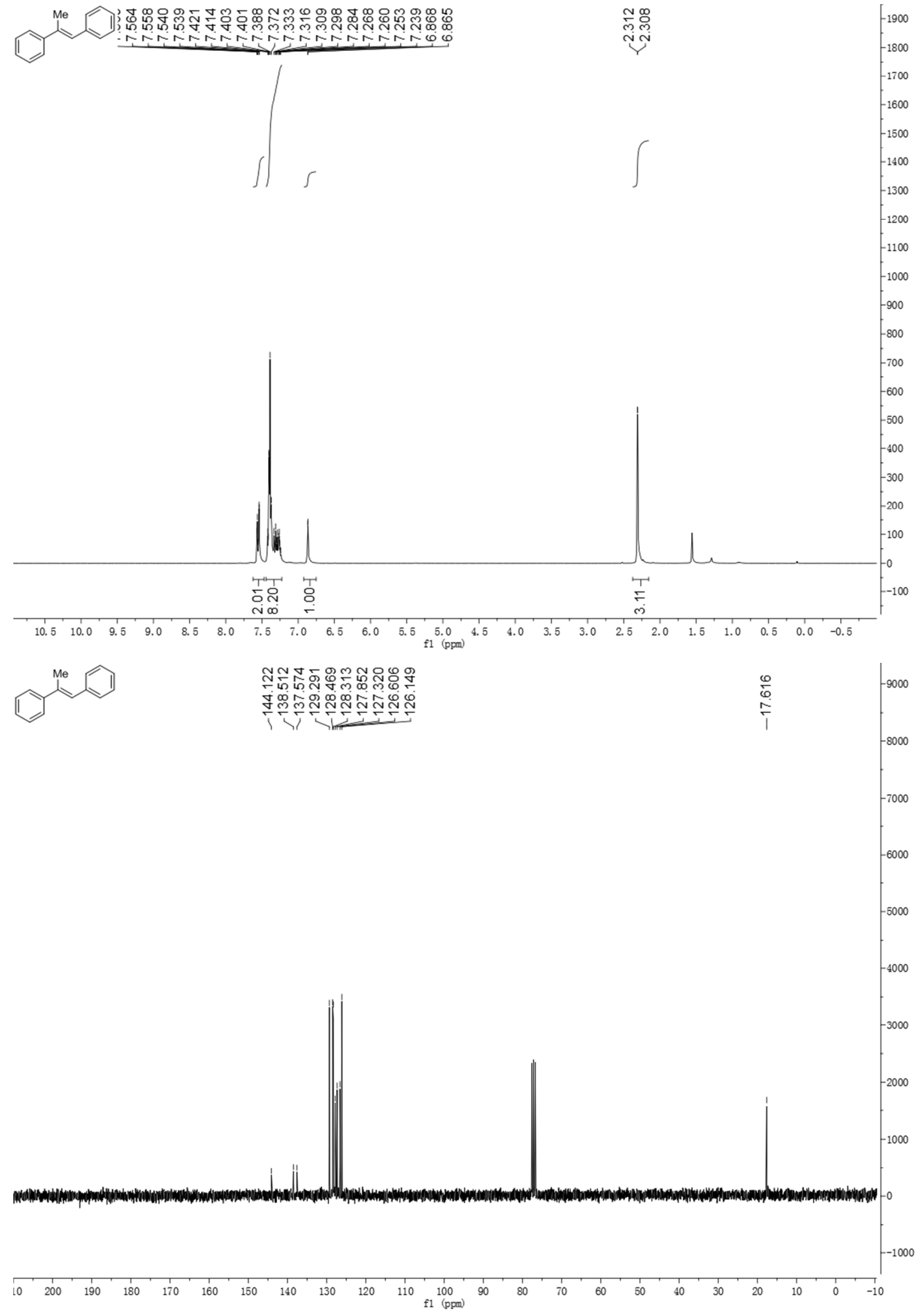




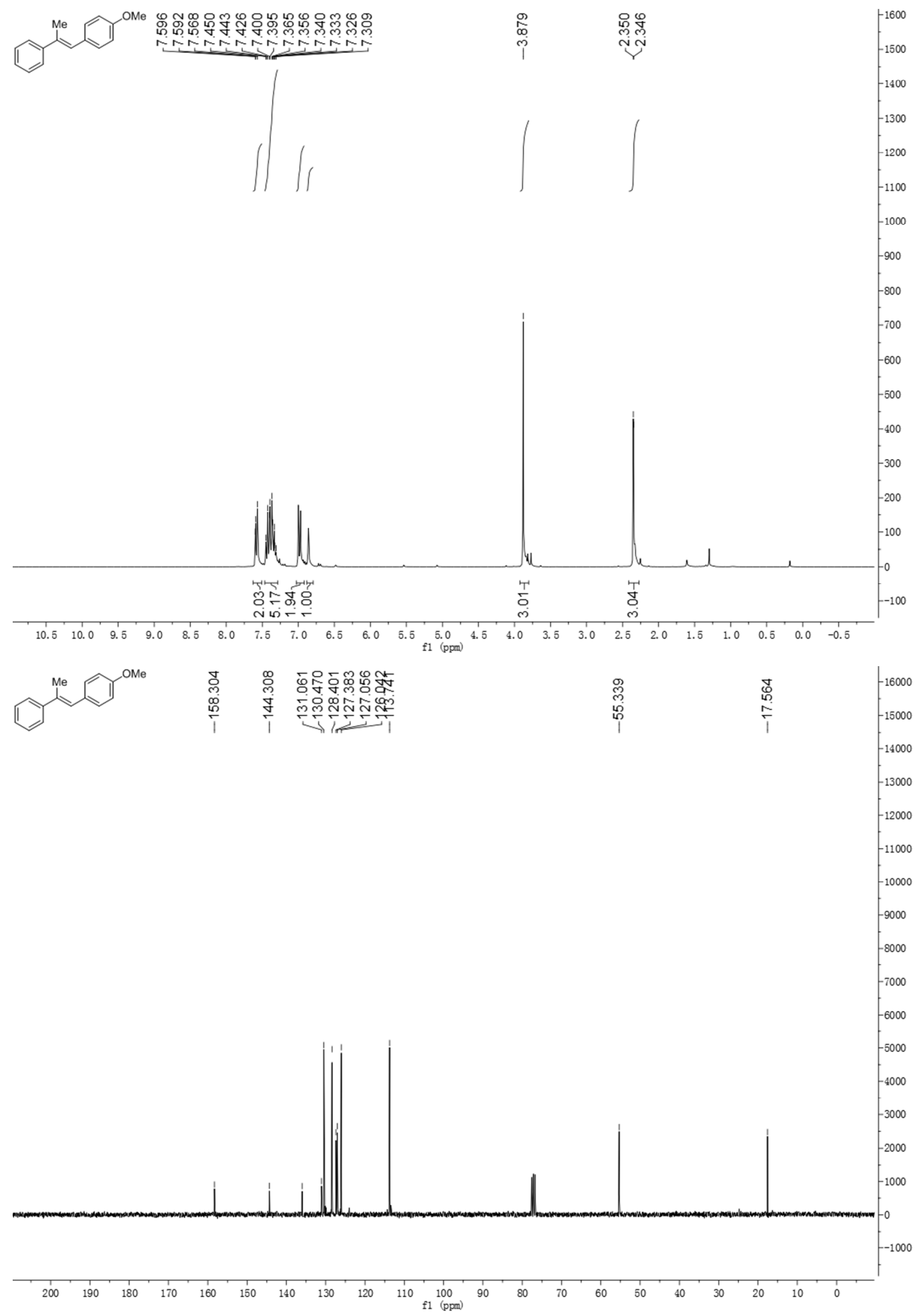




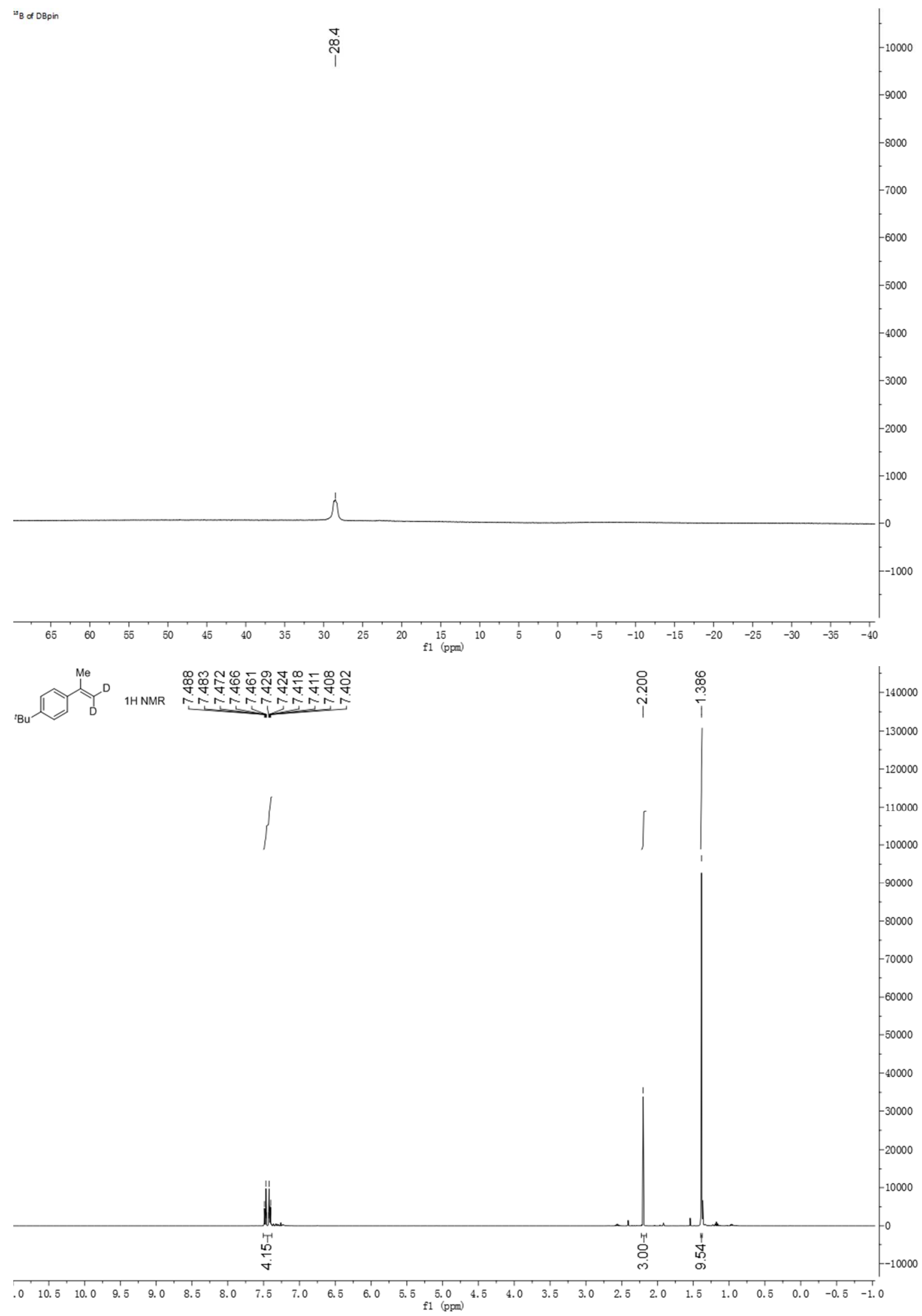


Wang, Wu, and GE, Supporting Information

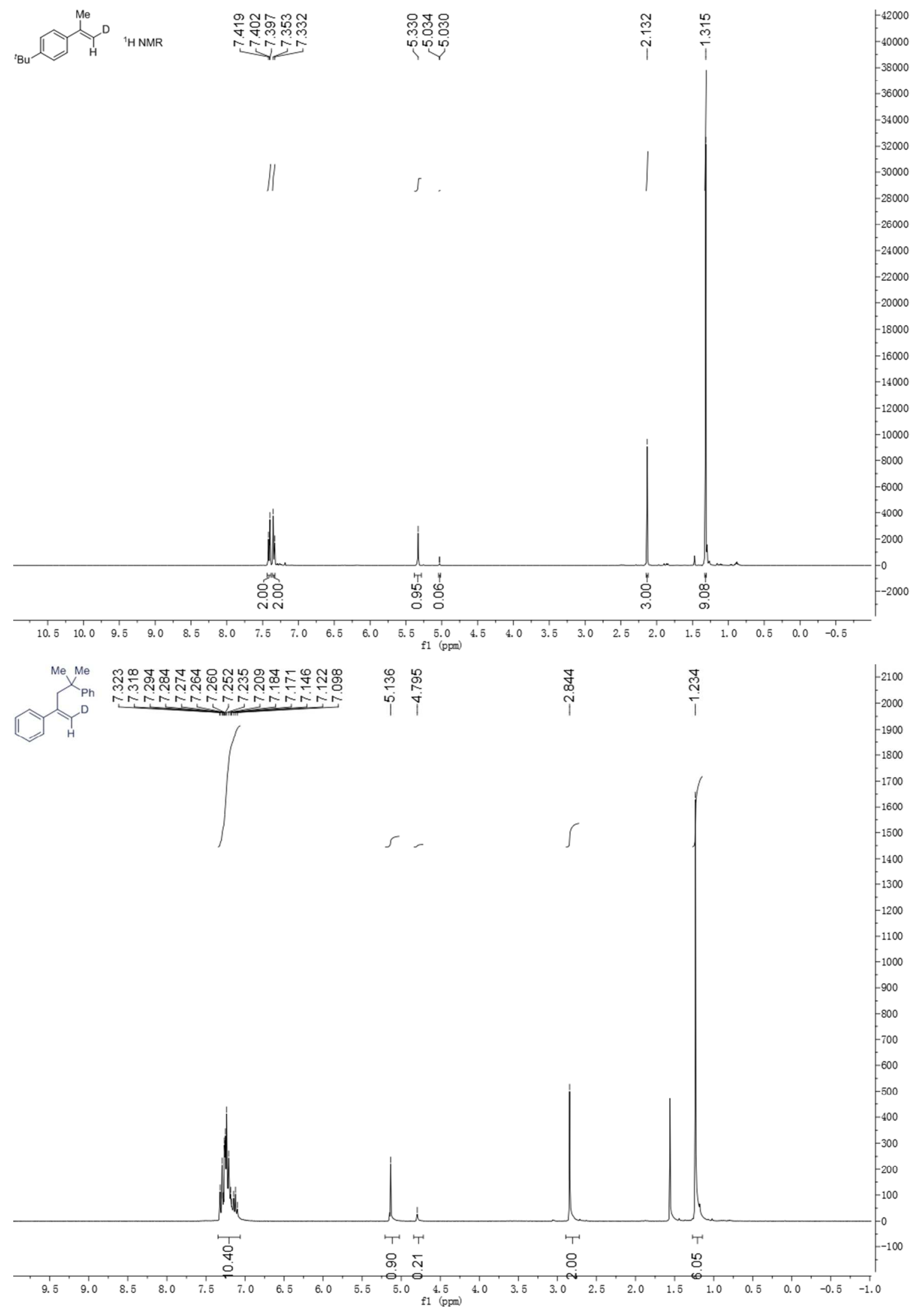




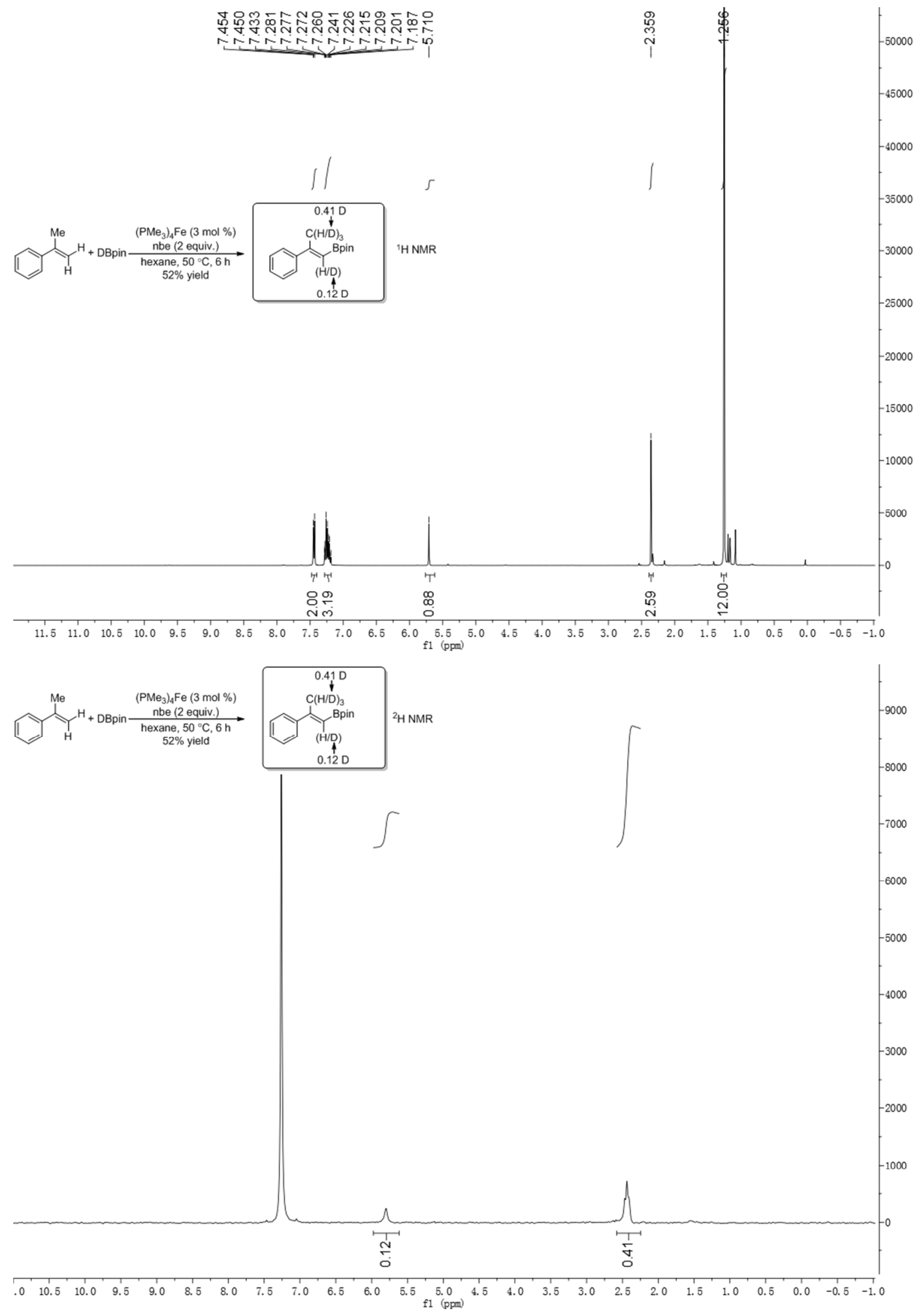



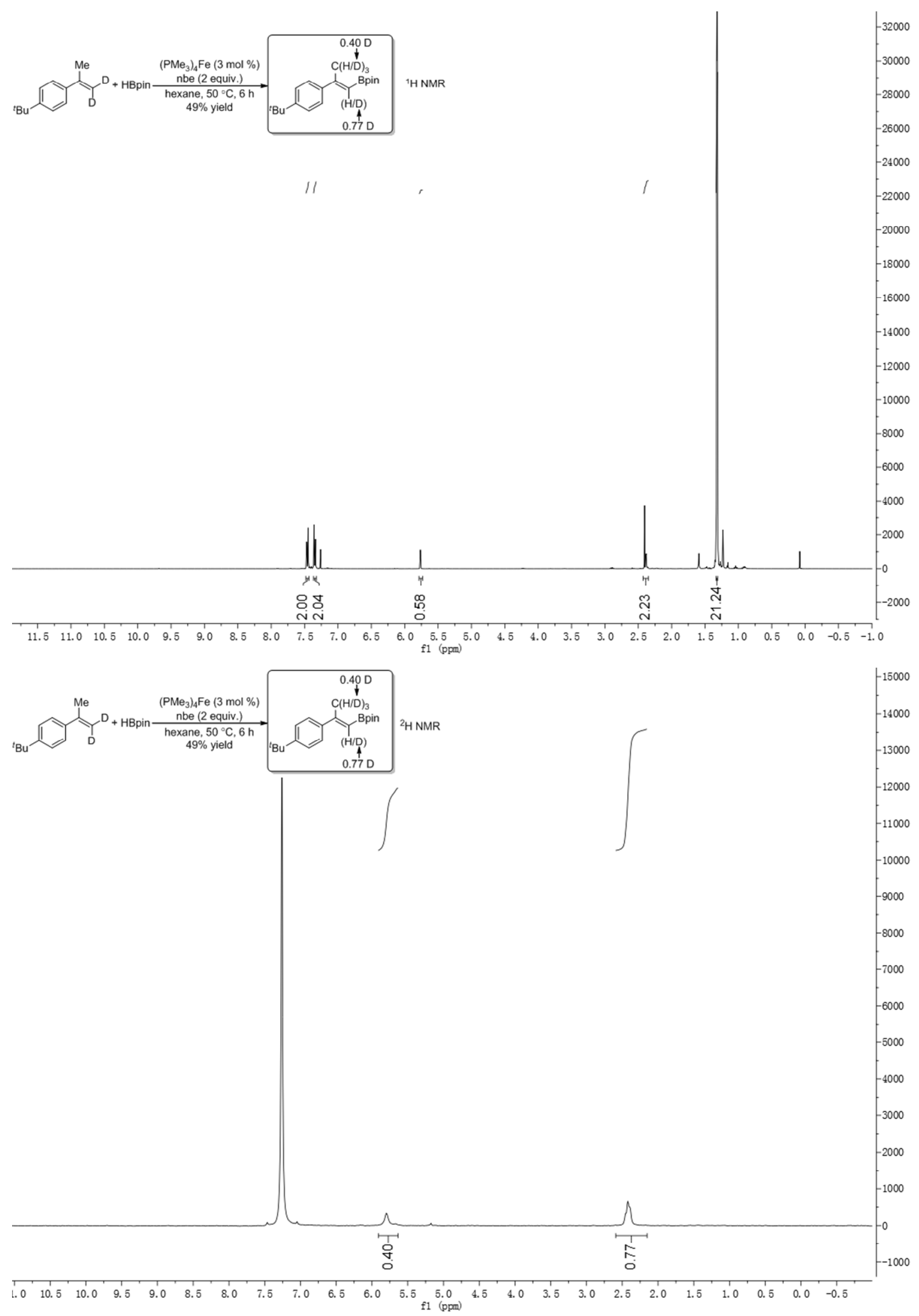


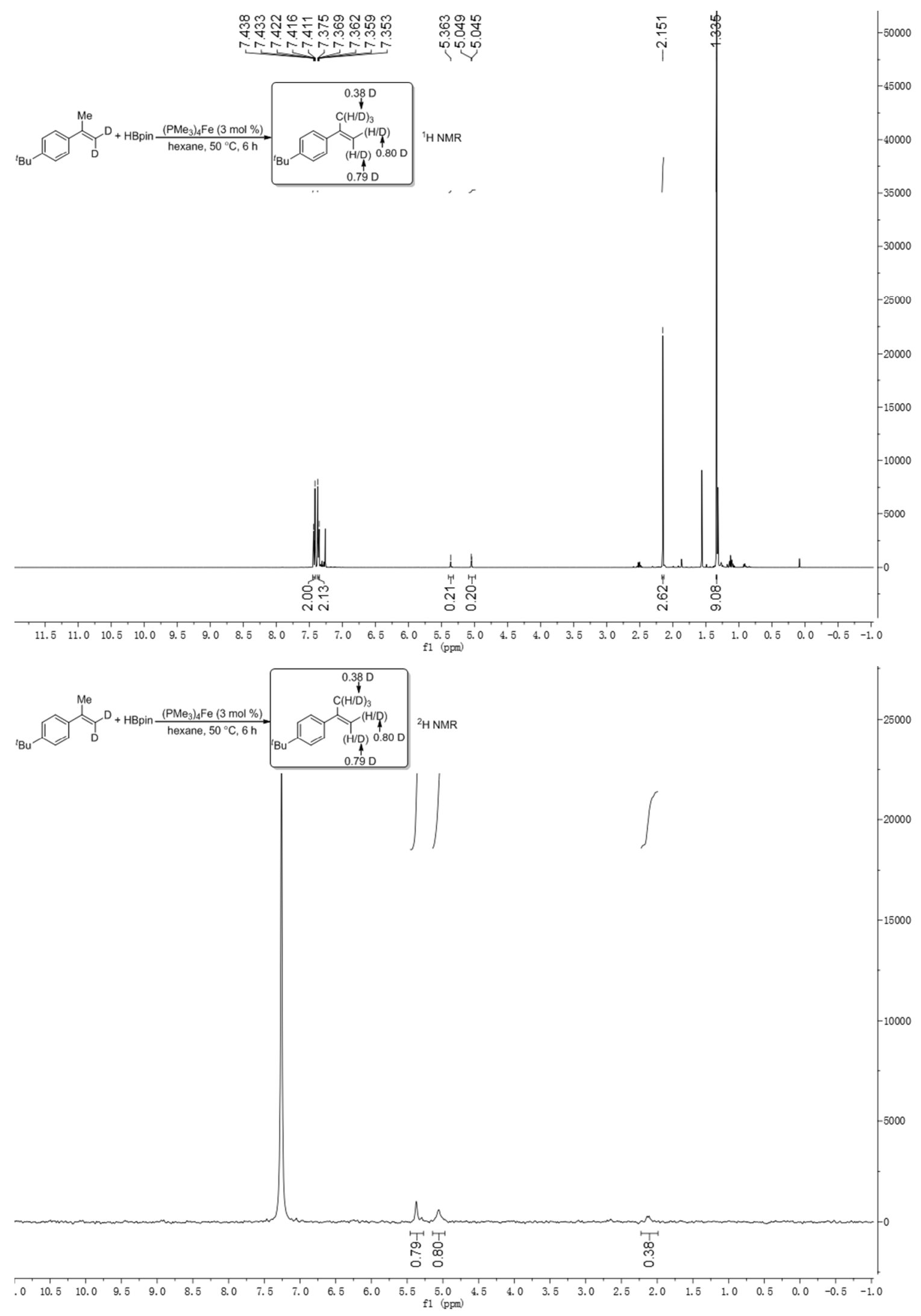


Wang, Wu, and GE, Supporting Information
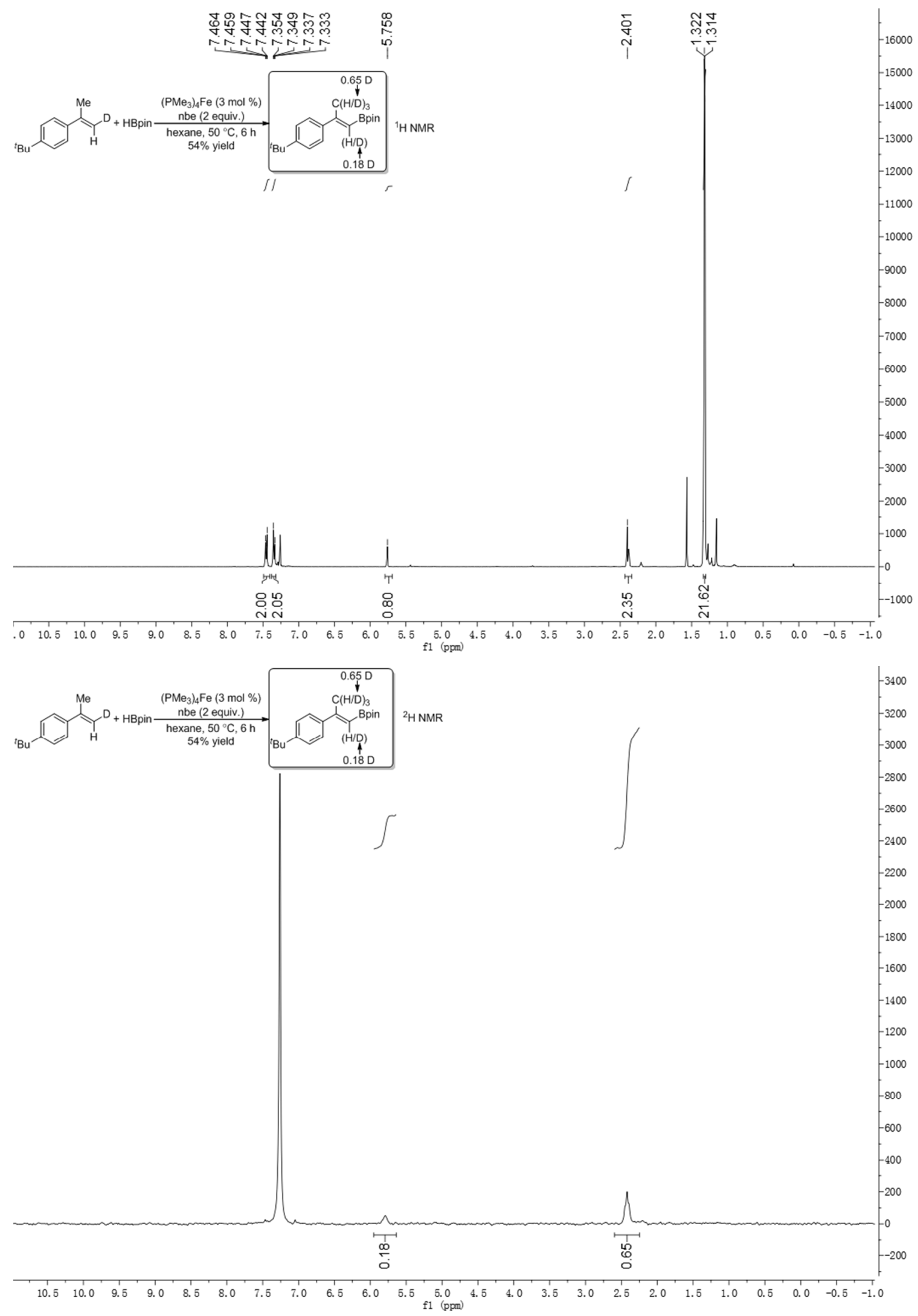
Wang, Wu, and GE, Supporting Information

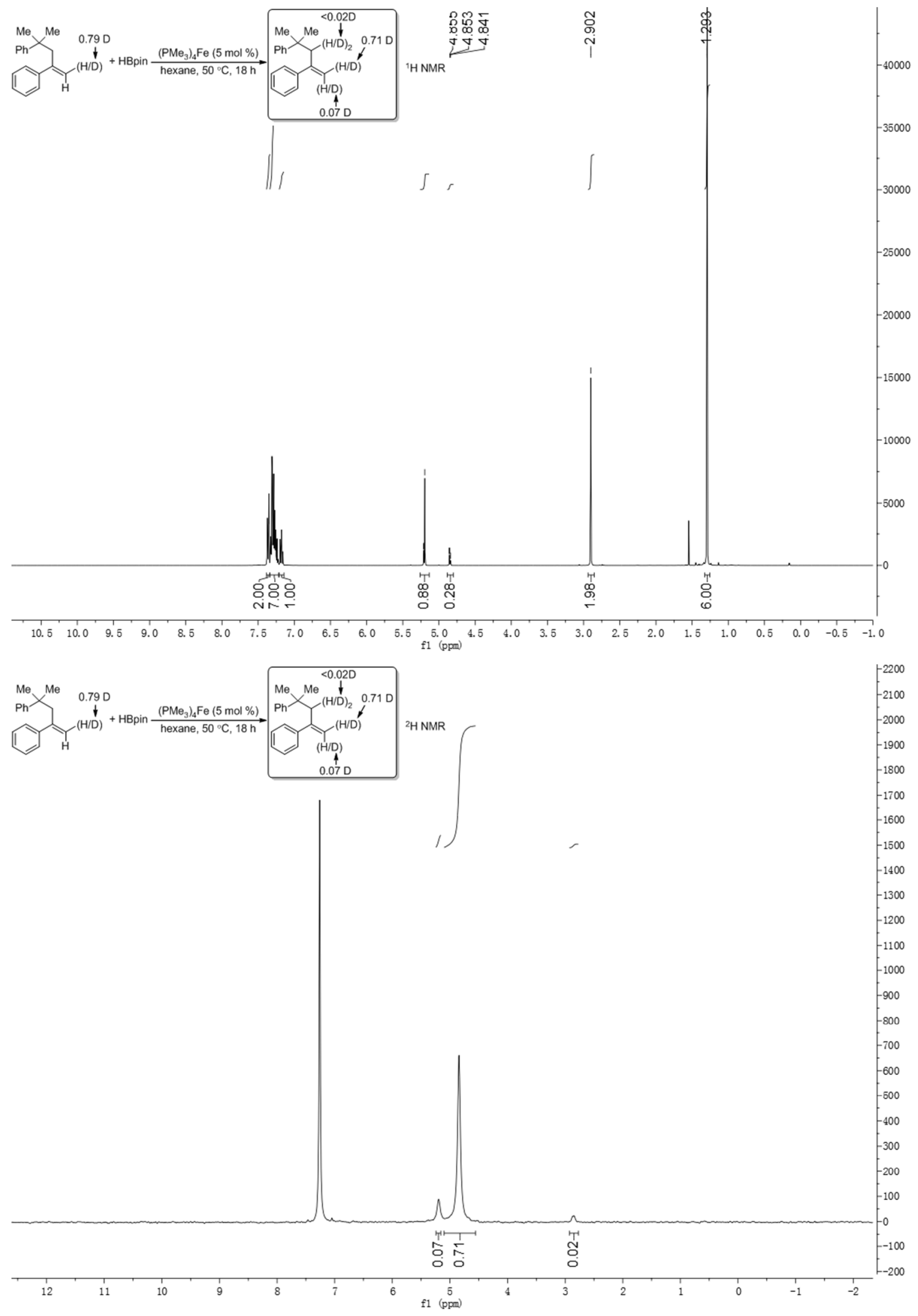




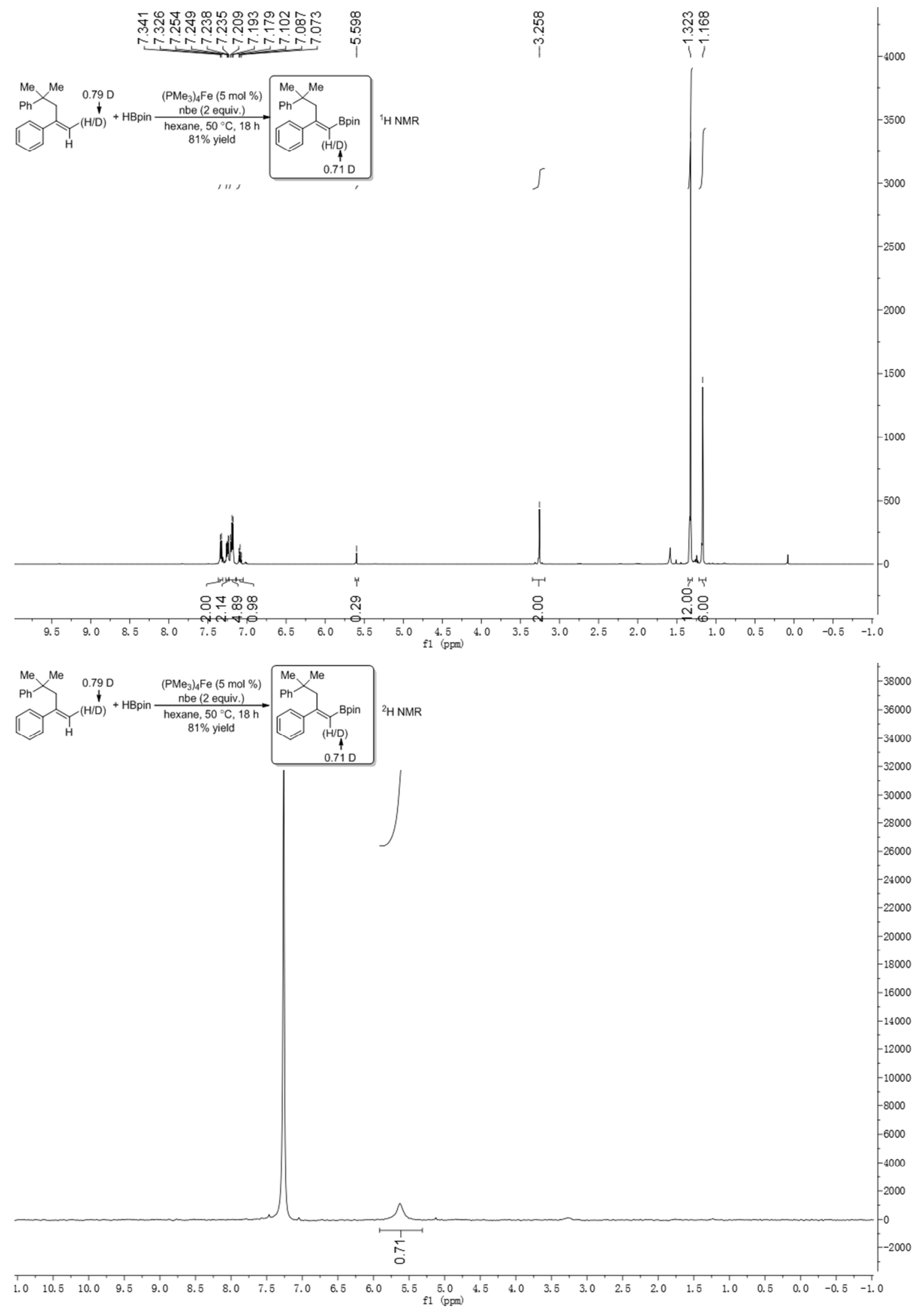


Wang, Wu, and GE, Supporting Information

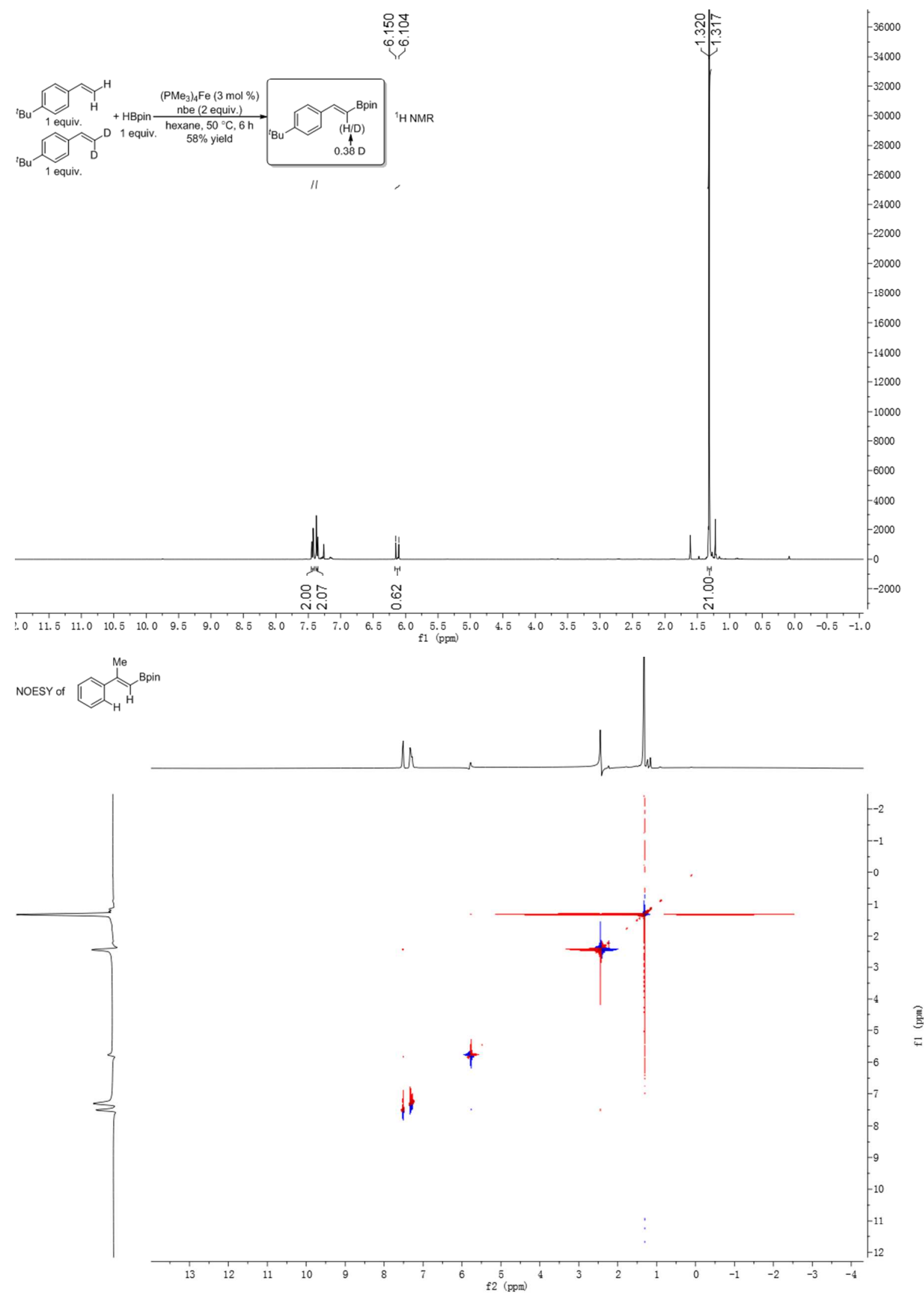



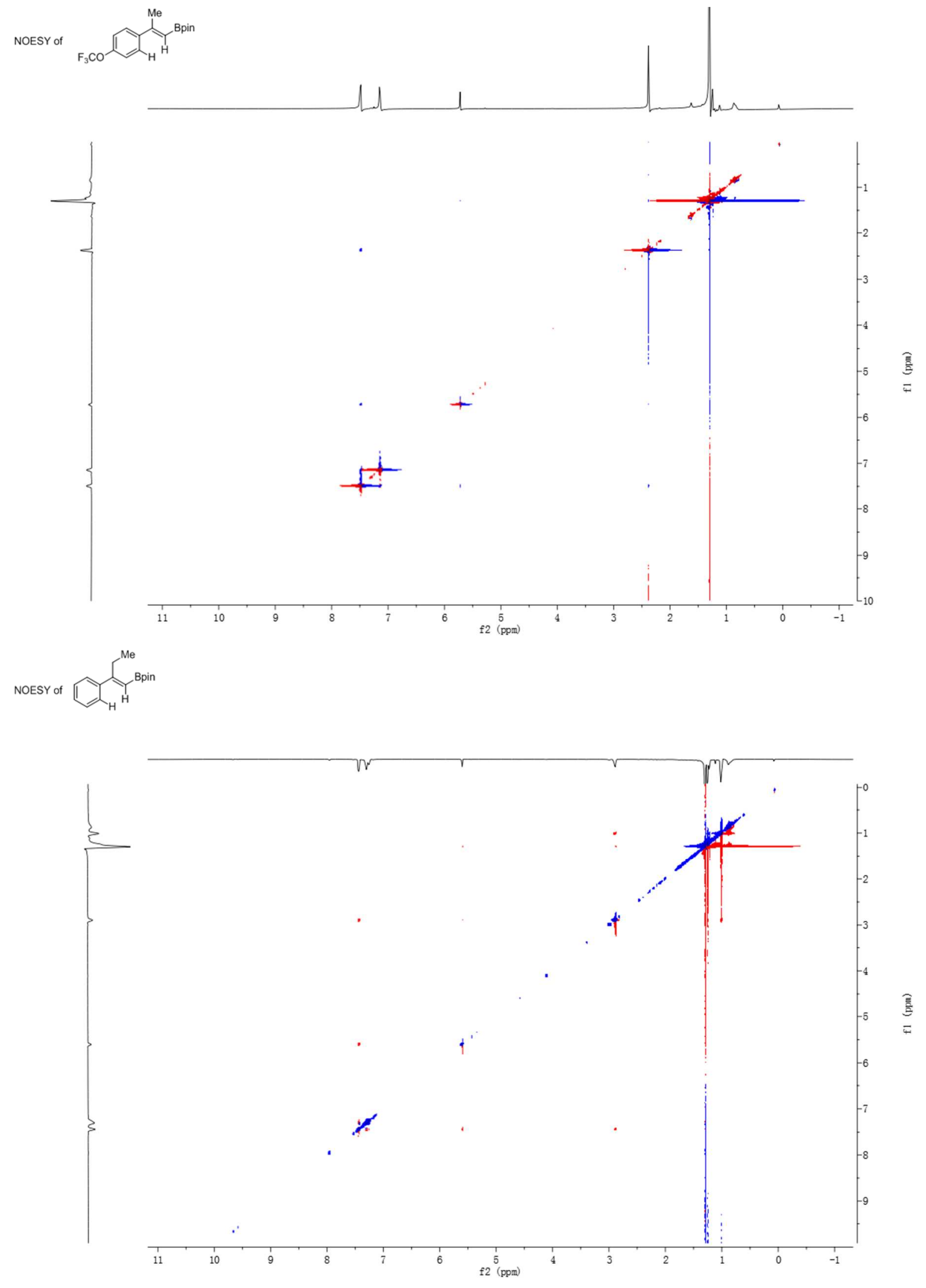
Wang, Wu, and GE, Supporting Information
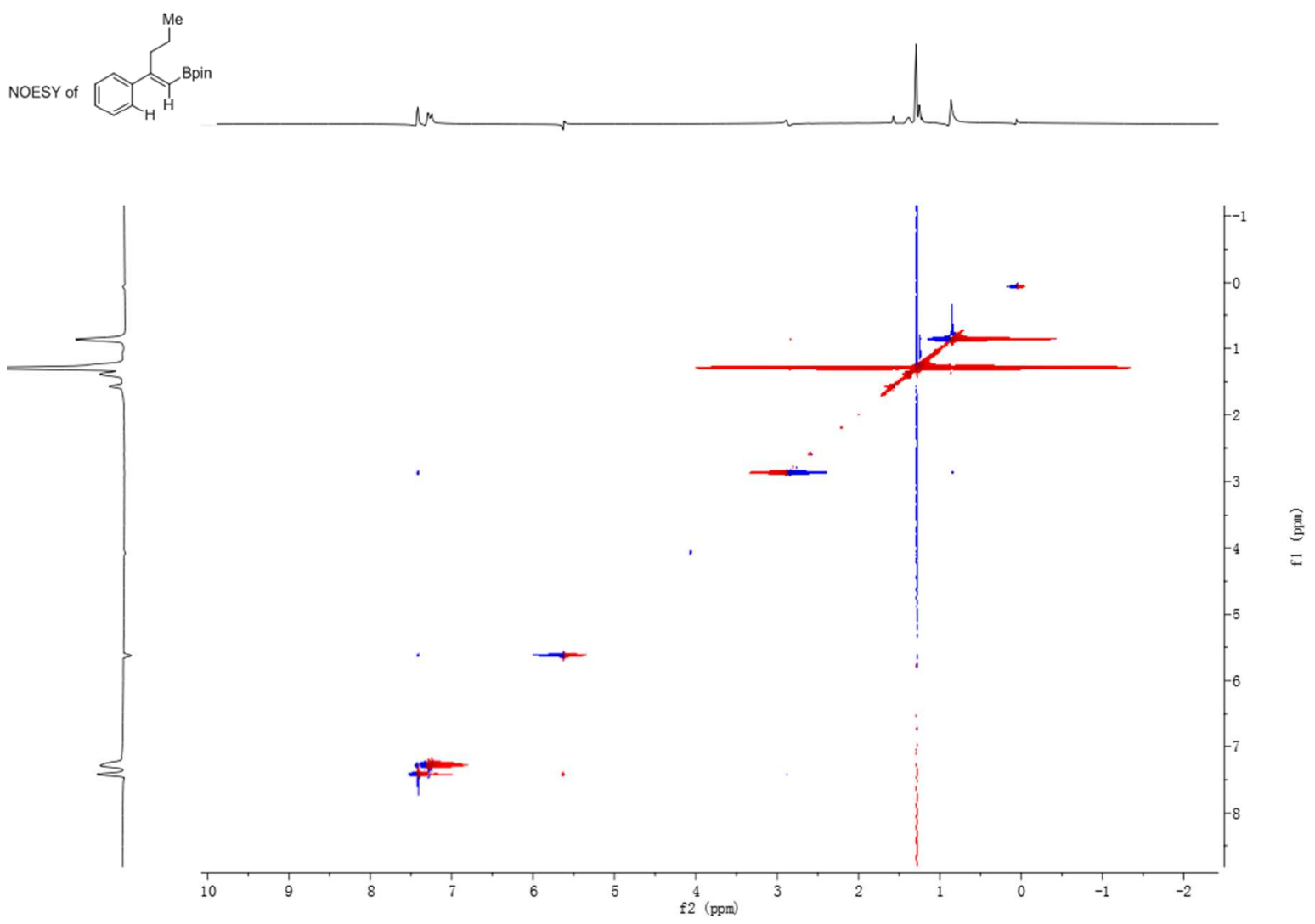